\title{
Semicartesian surfaces and the relaxed area of maps from the plane to the plane with a line discontinuity
}

\author{
Giovanni Bellettini ${ }^{1,2}$ - Maurizio Paolini ${ }^{3}$. \\ Lucia Tealdi ${ }^{4}$
}

Received: 7 September 2015 / Accepted: 27 January 2016 / Published online: 18 February 2016

(C) Fondazione Annali di Matematica Pura ed Applicata and Springer-Verlag Berlin Heidelberg 2016

\begin{abstract}
In this paper, we estimate the area of the graph of a map $\mathbf{u}: \Omega \subset \mathbb{R}^{2} \rightarrow \mathbb{R}^{2}$ discontinuous on a segment $J_{\mathbf{u}}$, with $J_{\mathbf{u}}$ either compactly contained in the bounded open set $\Omega$, or starting and ending on $\partial \Omega$. We characterize $\overline{\mathcal{A}}^{\infty}(\mathbf{u}, \Omega)$, the relaxed area functional in a sort of uniform convergence, in terms of the infimum of the area of those surfaces in $\mathbb{R}^{3}$ spanning the graphs of the traces of $\mathbf{u}$ on the two sides of $J_{\mathbf{u}}$ and having what we have called a semicartesian structure. We exhibit examples showing that $\overline{\mathcal{A}}(\mathbf{u}, \Omega)$, the relaxed area in $L^{1}\left(\Omega ; \mathbb{R}^{2}\right)$, may depend on the values of $\mathbf{u}$ far from $J_{\mathbf{u}}$ and also on the relative position of $J_{\mathbf{u}}$ with respect to $\partial \Omega$. These examples confirm the highly non-local behavior of $\overline{\mathcal{A}}(\mathbf{u}, \cdot)$ and justify the interest in the study of $\overline{\mathcal{A}}^{\infty}$. Finally we prove that $\overline{\mathcal{A}}(\mathbf{u}, \cdot)$ is not subadditive for a rather large class of discontinuous maps $\mathbf{u}$.
\end{abstract}

Keywords Relaxed area functional - Area of graphs - Semicartesian surfaces

Mathematics Subject Classification 49J45 - 49Q15 · 49Q20

Lucia Tealdi

1tealdi@sissa.it

Giovanni Bellettini

belletti@mat.uniroma2.it

Maurizio Paolini

paolini@dmf.unicatt.it

1 Dipartimento di Matematica, Università di Roma Tor Vergata, via della Ricerca Scientifica 1, 00133

Roma, Italy

2 INFN Laboratori Nazionali di Frascati, Frascati, Italy

3 Dipartimento di Matematica, Università Cattolica "Sacro Cuore", via Trieste 17, 25121 Brescia, Italy

4 SISSA, via Bonomea 265, 34136 Trieste, Italy 


\section{Introduction}

Given a bounded open set $\Omega \subset \mathbb{R}^{2}=\mathbb{R}_{(x, y)}^{2}$ and a map $\mathbf{v}:=\left(v_{1}, v_{2}\right): \Omega \rightarrow \mathbb{R}^{2}=\mathbb{R}_{(\xi, \eta)}^{2}$ of class $\mathcal{C}^{1}$, the (nonparametric) area functional is defined as

$$
\mathcal{A}(\mathbf{v}, \Omega):=\int_{\Omega}|\mathcal{M}(\nabla \mathbf{v})| \mathrm{d} x \mathrm{~d} y,
$$

where $\nabla \mathbf{v}$ is the Jacobian matrix of $\mathbf{v}, \mathcal{M}(\zeta)$ is the vector of $\mathbb{R}^{6}$ having as entries the determinant of all minors ${ }^{1}$ of the $(2 \times 2)$-matrix $\zeta$, and $|\cdot|$ denotes the Euclidean norm: hence, $|\mathcal{M}(\nabla \mathbf{v})|=\sqrt{1+\left|\nabla v_{1}\right|^{2}+\left|\nabla v_{2}\right|^{2}+\left(\partial_{x} v_{1} \partial_{y} v_{2}-\partial_{y} v_{1} \partial_{x} v_{2}\right)^{2}}$.

The functional $\mathcal{A}(\cdot, \Omega)$ is polyconvex $[5]$ and $\mathcal{A}(\mathbf{v}, \Omega)$ is the area of

$$
\operatorname{graph}(\mathbf{v}):=\{(x, y, \xi, \eta):(x, y) \in \Omega,(\xi, \eta)=\mathbf{v}(x, y)\} \subset \mathbb{R}^{4}:=\mathbb{R}_{(x, y)}^{2} \times \mathbb{R}_{(\xi, \eta)}^{2},
$$

a smooth two-dimensional manifold of codimension two.

For the purposes of the modern calculus of variations, it is useful to extend the area functional also to non-smooth maps. A rather natural idea consists in considering its $L^{1}$-lower semicontinuous envelope (or $L^{1}$-relaxed functional) $[1,7,9,10]$; thus, for any $\mathbf{v} \in L^{1}\left(\Omega ; \mathbb{R}^{2}\right)$, we set

$$
\overline{\mathcal{A}}(\mathbf{v}, \Omega):=\inf \left\{\liminf _{h \rightarrow+\infty} \mathcal{A}\left(\mathbf{v}_{h}, \Omega\right)\right\},
$$

where the infimum is taken among all sequences $\left(\mathbf{v}_{h}\right) \subset \mathcal{C}^{1}\left(\Omega ; \mathbb{R}^{2}\right)$ converging to $\mathbf{v}$ in $L^{1}\left(\Omega ; \mathbb{R}^{2}\right)$. The aim of the present paper is to study $\overline{\mathcal{A}}(\mathbf{v}, \Omega)$ for certain classes of non-smooth maps v. As we shall see, another (i.e., with respect to a different and stronger convergence) relaxed functional will be of interest, in this two-codimensional situation.

In [1, Theorem 3.7] it is proven that the domain of $\overline{\mathcal{A}}(\cdot, \Omega)$ is contained ${ }^{2}$ in the space $\operatorname{BV}\left(\Omega ; \mathbb{R}^{2}\right)$ of maps with bounded variation in $\Omega$, and for any $\mathbf{v} \in \operatorname{BV}\left(\Omega ; \mathbb{R}^{2}\right)$ it turns out that

$$
\overline{\mathcal{A}}(\mathbf{v}, \Omega) \geq \int_{\Omega}|\mathcal{M}(\nabla \mathbf{v})| \mathrm{d} x \mathrm{~d} y+\left|D^{s} \mathbf{v}\right|(\Omega),
$$

where $\nabla \mathbf{v}$ and $D^{S} \mathbf{v}$ denote the absolutely continuous and the singular part of the distributional gradient $D \mathbf{v}$, respectively. Moreover, in [1, Theorem 6.4] the subset of $\operatorname{BV}\left(\Omega ; \mathbb{R}^{2}\right)$ of all maps $\mathbf{v}$ for which

$$
\overline{\mathcal{A}}(\mathbf{v}, \Omega)=\int_{\Omega}|\mathcal{M}(\nabla \mathbf{v})| \mathrm{d} x \mathrm{~d} y<+\infty
$$

is characterized; from now on we shall use the symbol $\mathcal{A}$ in place of $\overline{\mathcal{A}}$ to denote the area of the graph of a map in this class. It is worth to notice that $\overline{\mathcal{A}}(\mathbf{v}, \Omega)=\int_{\Omega}|\mathcal{M}(\nabla \mathbf{v})| \mathrm{d} x \mathrm{~d} y<+\infty$ for every $\mathbf{v} \in H^{1}\left(\Omega ; \mathbb{R}^{2}\right)$.

One of the major issues on the functional $\overline{\mathcal{A}}(\mathbf{v}, \cdot)$ is its non-subadditivity [7]. In [1, Theorems 4.1 and 5.1] the authors exhibit two examples of maps $\mathbf{v}$ for which there exist three bounded open sets $\Omega_{1}, \Omega_{2}$, and $\Omega_{3}$ such that $\Omega_{3} \subset \subset \Omega_{1} \cup \Omega_{2}$ and

$$
\overline{\mathcal{A}}\left(\mathbf{v}, \Omega_{3}\right)>\overline{\mathcal{A}}\left(\mathbf{v}, \Omega_{1}\right)+\overline{\mathcal{A}}\left(\mathbf{v}, \Omega_{2}\right) .
$$

\footnotetext{
1 Including the minor of order 0 , whose determinant is by definition taken equal 1.

2 This inclusion is strict. For example, consider the map $\mathbf{u}(\mathbf{x})=\frac{\mathbf{x}}{|\mathbf{x}|^{3 / 2}}$, for $\mathbf{x}$ in the unit disk $B_{1}((1,0))$ centered at $(1,0)$. Since $\mathbf{u} \in \mathcal{C}^{1}\left(B_{1}((1,0)) ; \mathbb{R}^{2}\right) \cap W^{1,1}\left(B_{1}((1,0)) ; \mathbb{R}^{2}\right)$, it belongs to $\operatorname{BV}\left(B_{1}((1,0)) ; \mathbb{R}^{2}\right)$. Nevertheless $\operatorname{det}(\nabla \mathbf{u})$ is not integrable, and thus $\overline{\mathcal{A}}\left(\mathbf{u}, B_{1}((1,0))=+\infty\right.$.
} 
In the first theorem $\mathbf{v}=\mathbf{u}_{T} \in \mathrm{BV}\left(\Omega ; \mathbb{R}^{2}\right)$, a piecewise constant map taking three noncollinear values around a triple point, while in the second one ${ }^{3} \mathbf{v}(x, y)=\mathbf{u}_{V}(x, y):=\frac{(x, y)}{|(x, y)|}$ (vortex map), and thus $\mathbf{v} \in W^{1, p}\left(\Omega ; \mathbb{R}^{2}\right)$ for any $p \in[1,2)$. Notice that inequality (1.1) implies that $\overline{\mathcal{A}}(\mathbf{v}, \Omega)$ cannot be written as an integral, over $\Omega$, of a local integrand, integrated with respect to some measure; recall that, on the contrary, this integral representation holds in codimension one (see $[6,11,12])$.

In [3] the authors provide an upper bound for $\overline{\mathcal{A}}\left(\mathbf{u}_{T}, \Omega\right)$ that improves the estimate of [1]. They are able to control the singular contribution of the relaxed area functional, namely

$$
\overline{\mathcal{A}}\left(\mathbf{u}_{T}, \Omega\right)-\int_{\Omega}\left|\mathcal{M}\left(\nabla \mathbf{u}_{T}\right)\right| \mathrm{d} x \mathrm{~d} y,
$$

through the area of a suitable graph-type area-minimizing two-dimensional surface of codimension one, entangled at the triple point with two other similar surfaces.

In [4], the authors explore further the idea of estimating the above-mentioned singular contribution through the area of solutions of a suitable Plateau's-type problem in $\mathbb{R}^{3}$. More specifically, they study the case of a map $\mathbf{u}$ that is regular enough out of a simple smooth jump curve $J_{\mathbf{u}}$ compactly contained in $\Omega$. Then they consider the closed curve $\Gamma=\Gamma[\mathbf{u}] \subset \mathbb{R}^{3}$, supposed to be simple, obtained as the union of the graphs of the traces of $\mathbf{u}$ on the two sides of $J_{\mathbf{u}}$; the regularity of $\mathbf{u}$ implies the existence of an area-minimizing immersion $X_{\min } \in$ $\mathcal{C}^{2}\left(\bar{B} ; \mathbb{R}^{3}\right) \cap \mathcal{C}^{\omega}\left(B ; \mathbb{R}^{3}\right)$, mapping the boundary of the unit disk $B$ monotonically onto $\Gamma$, see for example [13] and [8]. In [4, Theorem 4.1] it is proven that, if $\Sigma_{\min }:=X_{\min }(B)$ admits a semicartesian parametrization, then

$$
\overline{\mathcal{A}}(\mathbf{u}, \Omega) \leq \int_{\Omega}|\mathcal{M}(\nabla \mathbf{u})| \mathrm{d} x \mathrm{~d} y+\mathcal{H}^{2}\left(\Sigma_{\text {min }}\right),
$$

where $\mathcal{H}^{2}$ denotes the two-dimensional Hausdorff measure. A map $\Phi: O \rightarrow \mathbb{R}^{3}, O \subset \mathbb{R}_{(t, s)}^{2}$ a bounded, open, connected and simply-connected set, is said to be semicartesian if it is the identity in the first coordinate, that is if $\Phi(t, s):=\left(t, \Phi_{2}(t, s), \Phi_{3}(t, s)\right)$, see Definition 2.3 for more details. In [4, Theorem 5.1] sufficient conditions on $\Gamma$, i.e., $\Gamma$ analytic with further non-degeneracy hypotheses, are given in order that $\Sigma_{\min }$ admits a semicartesian parametrization, and it is also conjectured that (1.2) could be an equality, at least when $J_{\mathbf{u}}$ is far enough from $\partial \Omega$. With the methods employed in [4], it seems not easy to weaken the analyticity and non-degeneracy assumptions (a part from the case when $\Gamma$ admits a graph-type solution of the corresponding Plateau's problem).

In this paper, we continue the analysis on the singular contribution of the nonparametric area functional for maps $\mathbf{u}$ having a line discontinuity $J_{\mathbf{u}}$, in terms of suitable area-minimizing semicartesian surfaces. We shall analyze both the case when $J_{\mathbf{u}}$ is compactly contained in $\Omega$ as well as when both its endpoints belong to $\partial \Omega$. These two cases are quite different from each other; in particular, as we shall see, the latter turns out to be related to minimal surfaces with a partially free boundary. Notice that we do not suppose a priori that the union of the graphs of the traces of $\mathbf{u}$ on $J_{\mathbf{u}}$ is a Jordan curve. Since we deal with maps with Lipschitz traces, even when $\Gamma$ is a Jordan curve, it does not satisfy the sufficient conditions of [4] that guarantee the existence of a semicartesian parametrization for a solution of the corresponding Plateau's problem.

Before stating our main results, we need to fix some notation and give some definitions, referring to Sect. 2 for the details.

3 The authors prove the result in dimension $n \geq 3$, but their proof holds also when $n=2$. 
Given two maps $\gamma^{ \pm} \in \operatorname{Lip}\left([a, b] ; \mathbb{R}^{2}\right)$, we consider their graphs $\Gamma^{ \pm} \subset \mathbb{R}^{3}:=\mathbb{R}_{t} \times$ $\mathbb{R}_{(\xi, \eta)}^{2}$. Let $\mathrm{R}:=(a, b) \times(-1,1) \subset \mathrm{R}_{(t, s)}^{2}$. We denote by semicart $\left(\mathrm{R} ; \Gamma^{-}, \Gamma^{+}\right)$the class of semicartesian maps on $\mathrm{R}$ spanning $\Gamma:=\Gamma^{-} \cup \Gamma^{+}$, that is the class of maps $\Phi \in H^{1}\left(\mathrm{R} ; \mathbb{R}^{3}\right)$ such that

$$
\Phi(t, s):=\left(t, \Phi_{2}(t, s), \Phi_{3}(t, s)\right), \quad \Phi(t, \pm 1)=\left(t, \gamma^{ \pm}(t)\right) .
$$

In particular, $\Phi(\mathrm{R})$ is a surface that intersects any plane $\{t\} \times \mathbb{R}_{(\xi, \eta)}^{2}$ in a (not necessarily simple) curve connecting the points $\left(t, \gamma^{-}(t)\right)$ and $\left(t, \gamma^{+}(t)\right)$, for any $t \in[a, b]$.

Since semicart $\left(\mathrm{R} ; \Gamma^{-}, \Gamma^{+}\right)$is non-empty (Lemma 2.8), we can define

$$
m\left(\mathrm{R} ; \Gamma^{-}, \Gamma^{+}\right):=\inf _{\Phi \in \operatorname{semicart}\left(\mathrm{R} ; \Gamma^{-}, \Gamma^{+}\right)} \int_{\mathrm{R}}\left|\partial_{t} \Phi \wedge \partial_{s} \Phi\right| \mathrm{d} t \mathrm{~d} s .
$$

If $\Gamma$ is a closed (not necessarily simple) curve, we can consider also another class of maps. Let

$$
D:=\left\{(t, s) \in \mathbb{R}^{2}: t \in(a, b), s \in\left(\sigma^{-}(t), \sigma^{+}(t)\right)\right\},
$$

with $\sigma^{ \pm} \in \operatorname{Lip}([a, b]), \sigma^{-}(t)<0$ and $\sigma^{+}(t)>0$ for $t \in(a, b)$ and $\sigma^{ \pm}(a)=0=$ $\sigma^{ \pm}(b)$. Then semicart $\left(D ; \Gamma^{-}, \Gamma^{+}\right)$denotes the class of semicartesian maps defined on $D$ and spanning $\Gamma:=\Gamma^{-} \cup \Gamma^{+}$, that is maps $\Phi \in H^{1}\left(D ; \mathbb{R}^{3}\right)$ such that

$$
\Phi(t, s):=\left(t, \Phi_{2}(t, s), \Phi_{3}(t, s)\right), \quad \Phi\left(t, \sigma^{ \pm}(t)\right)=\left(t, \gamma^{ \pm}(t)\right) .
$$

For such a $\Phi$, the image $\Phi(D)$ is a surface whose intersection with any plane $\{t\} \times \mathbb{R}_{(\xi, \eta)}^{2}$, $t$ belonging to the open interval $(a, b)$, is a curve connecting $\left(t, \gamma^{-}(t)\right)$ and $\left(t, \gamma^{+}(t)\right)$, but whose intersection with the plane $\{a\} \times \mathbb{R}_{(\xi, \eta)}^{2}$ (resp. with $\{b\} \times \mathbb{R}_{(\xi, \eta)}^{2}$ ) is the singleton $\left(a, \gamma^{-}(a)\right)$ (resp. $\left.\left(b, \gamma^{-}(b)\right)\right)$.

Also semicart $\left(D ; \Gamma^{-}, \Gamma^{+}\right)$is non-empty and then we can define

$$
m\left(D ; \Gamma^{-}, \Gamma^{+}\right):=\inf _{\Phi \in \operatorname{semicart}\left(D ; \Gamma^{-}, \Gamma^{+}\right)} \int_{D}\left|\partial_{t} \Phi \wedge \partial_{s} \Phi\right| \mathrm{d} t \mathrm{~d} s .
$$

We observe that when $\Gamma$ is closed, $m\left(\mathrm{R} ; \Gamma^{-}, \Gamma^{+}\right) \leq m\left(D ; \Gamma^{-}, \Gamma^{+}\right)$, since a surface that is image of a map in semicart $\left(D ; \Gamma^{-}, \Gamma^{+}\right)$can be obtained also as the image of a map in semicart (R; $\left.\Gamma^{-}, \Gamma^{+}\right)$(see (2.6)). If $\Gamma$ is closed and simple we could ask about the relations between $m\left(D ; \Gamma^{-}, \Gamma^{+}\right), m\left(\mathrm{R} ; \Gamma^{-}, \Gamma^{+}\right)$, and $a(\Gamma)$, the area of a solution of the classical Plateau's problem for $\Gamma$. In general $a(\Gamma) \leq m\left(D ; \Gamma^{-}, \Gamma^{+}\right)$, but one could expect also that the equal sign holds (see Remark 2.11), while we exhibit in Example 2.13 a curve $\Gamma$ for which

$$
m\left(\mathrm{R} ; \Gamma^{-}, \Gamma^{+}\right)<a(\Gamma) .
$$

In the study of the $L^{1}$-lower semicontinuous envelope of the area functional $\mathcal{A}(\cdot, \Omega)$, it would be important to have an $L^{1}$-lower semicontinuity result for $m(\mathrm{R} ; \cdot, \cdot)$, compare also with Remark 4.7. More precisely, if $\Gamma^{ \pm}:=\operatorname{graph}\left(\gamma^{ \pm}\right), \Gamma_{h}^{ \pm}:=\operatorname{graph}\left(\gamma_{h}^{ \pm}\right)$with $\gamma^{ \pm}, \gamma_{h}^{ \pm} \in$ $\operatorname{Lip}\left([a, b] ; \mathbb{R}^{2}\right)$, it would be desirable to prove that

$$
m\left(\mathrm{R} ; \Gamma^{-}, \Gamma^{+}\right) \leq \liminf _{h \rightarrow+\infty} m\left(\mathrm{R} ; \Gamma_{h}^{-}, \Gamma_{h}^{+}\right)
$$

whenever $\gamma_{h}^{ \pm} \rightarrow \gamma^{ \pm}$in $L^{1}\left((a, b) ; \mathbb{R}^{2}\right)$. We are able to prove (1.4) only under the further assumption that

$$
\sup _{h \in \mathbb{N}}\left\|\dot{\gamma}_{h}^{ \pm}\right\|_{L^{\infty}\left((a, b) ; \mathbb{R}^{2}\right)}<+\infty
$$


This is, in some sense, coherent with the lower semicontinuity of $a(\cdot)$ with respect to the Fréchet convergence (see $[8,13, \S 301]$ ); we shall show that $a(\cdot)$ is not lower semicontinuous with respect to the $L^{1}$-convergence, see Example 4.8 for the details. Proving the validity of (1.4) without assuming (1.5) seems not to be easy and would imply a characterization of $\overline{\mathcal{A}}(\mathbf{u}, \Omega)$ for certain non-smooth maps $\mathbf{u}$. The lack of a proof of (1.4) under the mere $L^{1}$-convergence forced us to define another extension of the functional $\mathcal{A}(\cdot, \Omega)$ with respect to a stronger notion of convergence, that we now describe.

Definition 1.1 (Uniform convergence out of a closed set) Let $\mathbf{v} \in \mathrm{BV}\left(\Omega ; \mathbb{R}^{2}\right)$ and $J \subset \Omega$ be a closed set with zero Lebesgue measure. A sequence $\left(\mathbf{v}_{h}\right) \subset L^{1}\left(\Omega ; \mathbb{R}^{2}\right)$ is said to converge to $\mathbf{v}$ uniformly out of $J$, if $\mathbf{v}_{h} \rightarrow \mathbf{v}$ uniformly in any compact set of $\Omega \backslash J$, as $h \rightarrow+\infty$.

We shall always consider maps $\mathbf{u} \in \operatorname{BV}\left(\Omega ; \mathbb{R}^{2}\right)$ so that, at any point of the (approximate) jump set [2], the approximate two-sided limits, denoted by $\mathbf{u}^{ \pm}$, coincide with the pointwise two-sided limits, and from now on, with a small abuse of notation, $J_{\mathbf{u}}$ stands for the closure in $\Omega$ of the set $\left\{(x, y) \in \Omega: \mathbf{u}^{-}(x, y) \neq \mathbf{u}^{+}(x, y)\right\}$.

We are now in a position to define another notion of relaxation of the area functional.

Definition 1.2 (The functional $\overline{\mathcal{A}}^{\infty}$ ) For any $\mathbf{v} \in \operatorname{BV}\left(\Omega ; \mathbb{R}^{2}\right)$ we define

$$
\overline{\mathcal{A}}^{\infty}(\mathbf{v}, \Omega):=\inf \left\{\liminf _{h \rightarrow+\infty} \mathcal{A}\left(\mathbf{v}_{h}, \Omega\right)\right\},
$$

where the infimum is taken among all sequences $\left(\mathbf{v}_{h}\right) \subset \mathcal{C}^{1}\left(\Omega ; \mathbb{R}^{2}\right)$ converging to $\mathbf{v}$ in $L^{1}\left(\Omega ; \mathbb{R}^{2}\right)$ and uniformly out of $J_{\mathbf{v}}$.

It is clear that

$$
\overline{\mathcal{A}}^{\infty}(\mathbf{v}, \Omega) \geq \overline{\mathcal{A}}(\mathbf{v}, \Omega), \quad \mathbf{v} \in \mathrm{BV}\left(\Omega ; \mathbb{R}^{2}\right) .
$$

For every $\mathbf{v}$ in the domain of the functional $\overline{\mathcal{A}}(\cdot, \Omega)$ it is also worth to define the singular parts

$$
\begin{gathered}
\overline{\mathcal{A}}_{s}(\mathbf{v}, \Omega):=\overline{\mathcal{A}}(\mathbf{v}, \Omega)-\int_{\Omega}|\mathcal{M}(\nabla \mathbf{v})| \mathrm{d} x \mathrm{~d} y, \\
\overline{\mathcal{A}}_{s}^{\infty}(\mathbf{v}, \Omega):=\overline{\mathcal{A}}^{\infty}(\mathbf{v}, \Omega)-\int_{\Omega}|\mathcal{M}(\nabla \mathbf{v})| \mathrm{d} x \mathrm{~d} y .
\end{gathered}
$$

The aim of this paper is to study the functionals $\overline{\mathcal{A}}_{s}$ and $\overline{\mathcal{A}}_{s}^{\infty}$, and also to characterize $\overline{\mathcal{A}}_{s}^{\infty}(\mathbf{u}, \Omega)$, for $\mathbf{u}$ in a suitable class of maps.

\subsection{Main results}

We shall consider two rather different cases: $\Omega$ and $\mathbf{u}$ satisfying either condition I or condition II (see Definitions 2.15 and 2.16, respectively). Condition I takes into account maps $\mathbf{u} \in$ $W^{1, \infty}\left(\Omega \backslash J_{\mathbf{u}} ; \mathbb{R}^{2}\right)$ having as jump set $J_{\mathbf{u}}$ a horizontal segment with both endpoints belonging to $\partial \Omega$; namely, the fracture "traverses" the whole domain $\Omega$. Condition II deals with maps $\mathbf{u} \in W^{1, \infty}\left(\Omega \backslash J_{\mathbf{u}} ; \mathbb{R}^{2}\right)$ with $J_{\mathbf{u}} \subset \subset \Omega$. We denote by

$$
\Gamma^{ \pm}[\mathbf{u}]
$$

the graphs of the traces of $\mathbf{u}$ on the two sides of $J_{\mathbf{u}}$ (Sect. 2.1).

Our first result characterizes the lower semicontinuous envelope of $\mathcal{A}$ in the sense of Definition 1.2. 
Theorem 1.3 (I: characterization of $\overline{\mathcal{A}}^{\infty}$ ) Let $\Omega$ and $\mathbf{u}$ satisfy condition I. Then

$$
\overline{\mathcal{A}}_{s}^{\infty}(\mathbf{u}, \Omega)=m\left(\mathrm{R} ; \Gamma^{-}[\mathbf{u}], \Gamma^{+}[\mathbf{u}]\right) .
$$

The inequality $\overline{\mathcal{A}}_{s}^{\infty}(\mathbf{u}, \Omega) \leq m\left(\mathrm{R} ; \Gamma^{-}[\mathbf{u}], \Gamma^{+}[\mathbf{u}]\right)$ is obtained using the same strategy proposed in [4], and it is proven in Proposition 3.1. The proof of the converse inequality (lower bound), presented in Sect. 4, is more interesting. As already noticed, in order to prove this inequality we use a lower semicontinuity result for $m(\mathrm{R} ; \cdot, \cdot)$ with respect to a convergence that is stronger than the one induced by the $L^{1}$-convergence, see Definition 1.1. Since we miss the proof of the $L^{1}\left((a, b) ; \mathbb{R}^{2}\right)$-lower semicontinuity of $m(\mathrm{R} ; \cdot, \cdot)$, we are not able to conclude the reasonable conjecture that $\overline{\mathcal{A}}_{s}(\mathbf{u}, \Omega)=m\left(\mathrm{R} ; \Gamma^{-}[\mathbf{u}], \Gamma^{+}[\mathbf{u}]\right)$, for $\Omega$ and $\mathbf{u}$ satisfying condition I and such that $\Gamma^{-}[\mathbf{u}] \cap \Gamma^{+}[\mathbf{u}]=\emptyset$.

When $\Omega$ and $\mathbf{u}$ satisfy condition II the situation is less clear. The proof of the next result is given in Proposition 5.1 and Theorem 6.1.

Theorem 1.4 (II: characterization of $\overline{\mathcal{A}}^{\infty}$ ) Let $\Omega$ and $\mathbf{u}$ satisfy condition II. Then

$$
\overline{\mathcal{A}}_{s}^{\infty}(\mathbf{u}, \Omega)=m\left(D ; \Gamma^{-}[\mathbf{u}], \Gamma^{+}[\mathbf{u}]\right) .
$$

What is interesting is that it may happen that

$$
\overline{\mathcal{A}}_{s}(\mathbf{u}, \Omega)<m\left(D ; \Gamma^{-}[\mathbf{u}], \Gamma^{+}[\mathbf{u}]\right),
$$

and thus there exist $\Omega$ and $\mathbf{u}$ for which

$$
\overline{\mathcal{A}}(\mathbf{u}, \Omega)<\overline{\mathcal{A}}^{\infty}(\mathbf{u}, \Omega) .
$$

In Sect. 7 we collect some examples proving that sequences $\left(\mathbf{u}_{h}\right)$ converging to $\mathbf{u}$ only in $L^{1}\left(\Omega ; \mathbb{R}^{2}\right)$ can be more "convenient" than any other sequence converging to $\mathbf{u}$ also uniformly out of $J_{\mathbf{u}}$. More specifically, in Sect. 7.1 we adapt to our case the construction used in [1, Lemma 5.3] concerning the area of the graph of the vortex map $\mathbf{u}_{V}$. The singular contribution of the area that we obtain can be interpreted as the area of a semicartesian parametrization defined on a suitable rectangle and spanning the graphs of the traces of $\mathbf{u}$ on a suitable extension $J_{\text {ext }}$ of $J_{\mathbf{u}}$ that reaches $\partial \Omega$. Example 7.4 proves that this construction can possibly provide an upper bound lower than $m\left(D ; \Gamma^{-}[\mathbf{u}], \Gamma^{+}[\mathbf{u}]\right)$. This suggests that it could be convenient to "extend" $J_{\mathbf{u}}$ up to the boundary by what we have called a virtual jump. As observed in Remark 7.5, we can manipulate the result in Example 7.4 and show that, if $J_{\mathbf{u}}$ has two connected components, the virtual jump could join one connected component to the other, instead of joining $J_{\mathbf{u}}$ to $\partial \Omega$. In Sect. 7.2 we exhibit an example where it is even more convenient to consider a virtual jump connecting an internal point of $J_{\mathbf{u}}$ to $\partial \Omega$.

All these examples reveal that the singular contribution of the area functional depends not only on the values of $\mathbf{u}$ near the jump set, but also on the values of $\mathbf{u}$ far from the jump and on the position of the jump with respect to $\partial \Omega$, confirming the deep non-local behavior of $\overline{\mathcal{A}}(\mathbf{u}, \cdot)$.

Our last result, Theorem 8.1 , concerns the non-subadditivity of $\overline{\mathcal{A}}$ with respect of the open set.

Theorem 1.5 There exist $\Omega$ and $\mathbf{u}$ satisfying condition I such that $\overline{\mathcal{A}}(\mathbf{u}, \cdot)$ is not subadditive.

The class of maps $\mathbf{u}$ for which Theorem 8.1 holds is rather large, and those maps can be written explicitly (see (8.1)). From Theorem 1.5, one deduces that the non-local character of $\overline{\mathcal{A}}(\mathbf{u}, \cdot)$ is not necessarily due to the presence of a vortex or of a triple junction, but it is a much more general fact. 
We underline that in order to prove Theorem 1.5 we do not use the results in Sects. 4-6, but only the upper bound in Proposition 3.1, and some (limited) results concerning cartesian currents (Proposition 8.6).

The plan of the paper is the following. In Sect. 2 we introduce the definitions and the main properties concerning the semicartesian setting, and we fix the hypotheses on the class of maps we consider in this work. In Sects. 3 and 4, we prove the upper and the lower bound that, coupled together, yield the proof of Theorem 1.3. Section 4 contains also the discussion on the semicontinuity of $m(\mathrm{R} ; \cdot, \cdot)$. Sections 5 and 6 deal with the case where $\Omega$ and $\mathbf{u}$ satisfy condition II and contain the proof of the upper and the lower bound needed to prove Theorem 1.4. In Sect. 7, we exhibit some examples of pairs $(\Omega, \mathbf{u})$ for which $\overline{\mathcal{A}}(\mathbf{u}, \Omega)<\overline{\mathcal{A}}^{\infty}(\mathbf{u}, \Omega)$. Finally in Sect. 8 we prove the non-subadditivity of $\overline{\mathcal{A}}(\mathbf{u}, \cdot)$ for a rather large class of maps.

\section{Semicartesian structure}

Let us start with some definitions. From now on we take $a, b \in \mathbb{R}=\mathbb{R}_{t}$, with $a<b$.

Definition 2.1 (Union of two graphs) Let $\Gamma \subset \mathbb{R}^{3}=\mathbb{R}_{t} \times \mathbb{R}_{(\xi, \eta)}^{2}$; we say that $\Gamma$ is union of two graphs on $[a, b]$ if $\Gamma=\Gamma^{-} \cup \Gamma^{+}$, where $\Gamma^{ \pm}:=\operatorname{graph}\left(\gamma^{ \pm}\right)$with $\gamma^{ \pm} \in \mathcal{C}\left([a, b] ; \mathbb{R}^{2}\right) \cap$ $\operatorname{Lip}_{\text {loc }}\left((a, b) ; \mathbb{R}^{2}\right)$. We say that $\Gamma$ is union of two Lipschitz graphs on $[a, b]$ if furthermore $\gamma^{ \pm} \in \operatorname{Lip}\left([a, b] ; \mathbb{R}^{2}\right)$.

Remark 2.2 Depending on the values of $\gamma^{ \pm}$at $t=a$ and $t=b, \Gamma$ could be either a closed curve, or an open curve, or the union of two open curves. Notice that we do not exclude that $\gamma^{-}(t)=\gamma^{+}(t)$ for some $t \in(a, b)$. We shall be mostly interested in the cases when either $\Gamma$ is closed, or when $\gamma^{-}(a) \neq \gamma^{+}(a)$ and $\gamma^{-}(b) \neq \gamma^{+}(b)$. The latter case will be related to a partially free boundary problem.

Definition 2.3 (Semicartesian map) A semicartesian map on $O$ is a continuous map $\Phi$ : $\bar{O} \rightarrow \mathbb{R}^{3}$ of the form

$$
\Phi(t, s)=(t, \phi(t, s))=\left(t, \phi_{1}(t, s), \phi_{2}(t, s)\right), \quad(t, s) \in O,
$$

where

$$
O:=\left\{(t, s) \in \mathbb{R}^{2}: \sigma^{-}(t)<s<\sigma^{+}(t), t \in(a, b)\right\},
$$

with $\sigma^{ \pm} \in \mathcal{C}([a, b]) \cap \operatorname{Lip}_{\text {loc }}((a, b))$ and $\sigma^{-}<\sigma^{+}$in $(a, b)$.

If we need to stress the dependence on the functions $\sigma^{ \pm}$, we shall use the notation $O=$ $\left[\left[\sigma^{-}, \sigma^{+}\right]\right]$.

Definition 2.4 (Semicartesian parametrizations) Given $\Gamma=\Gamma^{-} \cup \Gamma^{+}$union of two graphs on $[a, b], \Gamma^{ \pm}:=\operatorname{graph}\left(\gamma^{ \pm}\right)$, a semicartesian parametrization spanning $\Gamma$ is a pair $(O, \Phi)$ where $O=\left[\left[\sigma^{-}, \sigma^{+}\right]\right]$and $\Phi$ is a semicartesian map on $O$ satisfying the boundary condition

$$
\Phi\left(t, \sigma^{ \pm}(t)\right)=\left(t, \gamma^{ \pm}(t)\right), \quad t \in[a, b] .
$$

We notice that, if $\gamma^{-}(a) \neq \gamma^{+}(a)$, the domain $O=\left[\left[\sigma^{-}, \sigma^{+}\right]\right]$of a semicartesian parametrization $(O, \Phi)$ spanning $\Gamma=\Gamma^{-} \cup \Gamma^{+}$has to satisfy $\sigma^{-}(a)<\sigma^{+}(a)$. Similarly, if $\gamma^{-}(b) \neq \gamma^{+}(b)$, necessarily $\sigma^{-}(b)<\sigma^{+}(b)$. On the other hand, if $\gamma^{-}(a)=\gamma^{+}(a)$, we can in principle choose either a domain $O$ such that $\sigma^{-}(a)=\sigma^{+}(a)$ or such that $\sigma^{-}(a)<\sigma^{+}(a)$. The trace on the plane $\{t=a\}$ of the image of $\Phi$ is, in the first case, just 
the point $\left(a, \gamma^{-}(a)\right)$; while in the second case, it is a not necessarily simple, closed, curve. Similar considerations are valid at $t=b$.

In the following, we shall need more regularity on $\Phi$, since we need the area of a semicartesian parametrization to be finite; in Sects. 3 and 5 we will need $\Phi$ and its derivatives to be square integrable, in order to build maps $\mathbf{u}_{h} \in H^{1}\left(\Omega ; \mathbb{R}^{2}\right)$. This is, in some sense, coherent also with the classical theory of Plateau's problem [8], where an area-minimizing immersion of the disk is found by minimizing the Dirichlet functional.

Finally we will fix special domains $O$. In Lemma 2.14 we will show that this can be done without loss of generality.

Definition 2.5 (The domains $\mathrm{R}$ and $D$ ) We set

$$
\mathrm{R}:=(a, b) \times(-1,1),
$$

namely $\mathrm{R}=\left[\left[\sigma_{\mathrm{R}}^{-}, \sigma_{\mathrm{R}}^{+}\right]\right]$, with $\sigma_{\mathrm{R}}^{-} \equiv-1$ and $\sigma_{\mathrm{R}}^{+} \equiv 1$.

We also fix two maps $\sigma^{ \pm} \in \operatorname{Lip}([a, b])$ so that $\sigma^{-}<\sigma^{+}$on $(a, b)$ and

- $\sigma^{-}(a)=\sigma^{+}(a)=0$ and $\sigma^{ \pm}(t)=\mathcal{O}(t-a)$, for $t \in(a, a+\delta), \delta>0$ small enough;

- $\sigma^{-}(b)=\sigma^{+}(b)=0$ and $\sigma^{ \pm}(t)=\mathcal{O}(b-t)$, for $t \in(b-\delta, b), \delta>0$ small enough, ${ }^{4}$

and we define

$$
D:=\left[\left[\sigma^{-}, \sigma^{+}\right]\right] .
$$

Definition 2.6 (The classes semicart) Let $\Gamma=\Gamma^{-} \cup \Gamma^{+}$be union of two Lipschitz graphs on $[a, b]$. We set

$$
\operatorname{semicart}\left(\mathrm{R} ; \Gamma^{-}, \Gamma^{+}\right):=\left\{\Phi \in H^{1}\left(\mathrm{R} ; \mathbb{R}^{3}\right):\right.
$$

$(\mathrm{R}, \Phi)$ semicartesian parametrization spanning $\Gamma\}$, $\operatorname{semicart}\left(D ; \Gamma^{-}, \Gamma^{+}\right):=\left\{\Phi \in H^{1}\left(D ; \mathbb{R}^{3}\right):\right.$

$(D, \Phi)$ semicartesian parametrization spanning $\Gamma\}$.

Remark 2.7 (Area integrand for semicartesian maps) For a semicartesian map $\Phi$ as in (2.1) belonging either to semicart $\left(\mathrm{R} ; \Gamma^{-}, \Gamma^{+}\right)$or to $\operatorname{semicart}\left(D ; \Gamma^{-}, \Gamma^{+}\right)$, we have

$$
\left|\partial_{t} \Phi \wedge \partial_{S} \Phi\right|=\sqrt{\left|\partial_{s} \phi\right|^{2}+\left(\partial_{t} \phi_{1} \partial_{s} \phi_{2}-\partial_{t} \phi_{2} \partial_{s} \phi_{1}\right)^{2}} .
$$

The area of a semicartesian parametrization is therefore

$$
\int\left|\partial_{t} \Phi \wedge \partial_{s} \Phi\right| \mathrm{d} t \mathrm{~d} s=\int \sqrt{\left|\partial_{s} \phi\right|^{2}+\left(\partial_{t} \phi_{1} \partial_{s} \phi_{2}-\partial_{t} \phi_{2} \partial_{s} \phi_{1}\right)^{2}} \mathrm{~d} t \mathrm{~d} s,
$$

where the domain of integration of the integrals is either $\mathrm{R}$ or $D$. If in particular $\phi_{1}(t, s)=$ $s$, the right hand side of (2.2) reduces obviously to $\sqrt{1+\left|\partial_{t} \phi_{2}\right|^{2}+\left|\partial_{s} \phi_{2}\right|^{2}}$, namely the integrand of the area functional in the one-codimensional cartesian case.

Notice that, if $\Gamma^{ \pm}=\operatorname{graph}\left(\gamma^{ \pm}\right)$with either $\gamma^{-}(a) \neq \gamma^{+}(a)$ or $\gamma^{-}(b) \neq \gamma^{+}(b)$, then the class semicart $\left(D ; \Gamma^{-}, \Gamma^{+}\right)$is empty.

Lemma 2.8 Let $\Gamma=\Gamma^{-} \cup \Gamma^{+}$be union of two Lipschitz graphs on $[a, b]$. Then

$$
\operatorname{semicart}\left(\mathrm{R} ; \Gamma^{-}, \Gamma^{+}\right) \neq \emptyset .
$$

If in addition $\Gamma$ is closed, then also semicart $\left(D ; \Gamma^{-}, \Gamma^{+}\right) \neq \emptyset$.

4 These growth assumptions are needed in order to prove that the linear interpolating map $\Phi_{\ell_{D}}$ defined in Lemma 2.8, see (2.3), has the right regularity. Lemma 2.14 shows that this assumption is not restrictive. 
Proof Write $\Gamma^{ \pm}=\operatorname{graph}\left(\gamma^{ \pm}\right)$. Let us define the following $\mathbb{R}^{2}$-valued Lipschitz continuous linear interpolating map:

$$
\ell_{\mathrm{R}}(t, s):=\frac{1-s}{2} \gamma^{-}(t)+\frac{1+s}{2} \gamma^{+}(t), \quad(t, s) \in \overline{\mathrm{R}} .
$$

Then $\ell_{\mathrm{R}}(t, \pm 1)=\gamma^{ \pm}(t)$; thus the map $\Phi_{\ell_{\mathrm{R}}}(t, s):=\left(t, \ell_{\mathrm{R}}(t, s)\right)$ belongs to $\operatorname{semicart}\left(\mathrm{R} ; \Gamma^{-}, \Gamma^{+}\right)$.

Now, suppose that $\gamma^{-}(a)=\gamma^{+}(a)$ and $\gamma^{-}(b)=\gamma^{+}(b)$ (i.e., $\Gamma$ is closed) and define

$$
\ell_{D}(t, s):= \begin{cases}\frac{\sigma^{+}(t)-s}{\sigma^{+}(t)-\sigma^{-}(t)} \gamma^{-}(t)+\frac{s-\sigma^{-}(t)}{\sigma^{+}(t)-\sigma^{-}(t)} \gamma^{+}(t) & (t, s) \in D, \\ \gamma^{ \pm}(t) & s=\sigma^{ \pm}(t), t \in[a, b] .\end{cases}
$$

Thus for $(t, s) \in D$

$$
\begin{aligned}
\partial_{t} \ell(t, s)= & \frac{\sigma^{+}(t)-s}{\sigma^{+}(t)-\sigma^{-}(t)} \dot{\gamma}^{-}(t)+\frac{s-\sigma^{-}(t)}{\sigma^{+}(t)-\sigma^{-}(t)} \dot{\gamma}^{+}(t) \\
& +\frac{\left(\gamma^{+}(t)-\gamma^{-}(t)\right)\left(\dot{\sigma}^{+}(t) \sigma^{-}(t)-\sigma^{+}(t) \dot{\sigma}^{-}(t)\right)}{\left(\sigma^{+}(t)-\sigma^{-}(t)\right)^{2}} \\
& -\frac{s\left(\gamma^{+}(t)-\gamma^{-}(t)\right)\left(\dot{\sigma}^{+}(t)-\dot{\sigma}^{-}(t)\right)}{\left(\sigma^{+}(t)-\sigma^{-}(t)\right)^{2}}, \\
\partial_{s} \ell(t, s)= & \frac{\gamma^{+}(t)-\gamma^{-}(t)}{\sigma^{+}(t)-\sigma^{-}(t)} .
\end{aligned}
$$

Since $\gamma^{ \pm} \in \operatorname{Lip}\left([a, b] ; \mathbb{R}^{2}\right)$, the properties on $\sigma^{ \pm}$in Definition 2.5 ensure that

$$
\left|\frac{\gamma^{+}(t)-\gamma^{-}(t)}{\sigma^{+}(t)-\sigma^{-}(t)}\right| \leq C<+\infty \quad t \in(a, a+\delta),
$$

and similarly for $t \in(b-\delta, b)$. Noticing also that $\left|\dot{\sigma}^{+} \sigma^{-}-\sigma^{+} \dot{\sigma}^{-}\right| \leq C\left(\sigma^{+}-\sigma^{-}\right)$ (for a possibly different positive constant $C$ ), and recalling that $\gamma^{ \pm}$and $\sigma^{ \pm}$are Lipschitz continuous, we get that $\partial_{t} \ell_{D}$ and $\partial_{s} \ell_{D}$ are bounded. It follows that the map

$$
\Phi_{\ell_{D}}(t, s):=\left(t, \ell_{D}(t, s)\right)
$$

belongs to $W^{1, \infty}\left(D ; \mathbb{R}^{3}\right)$, and in particular to semicart $\left(D ; \Gamma^{-}, \Gamma^{+}\right)$.

Remark 2.9 If $\gamma^{+}(t)=\gamma^{-}(t)$ for some $t \in(a, b), \Phi_{\ell_{\mathrm{R}}}$ (resp. $\Phi_{\ell_{D}}$ ) maps the segment $\{t\} \times[-1,1]\left(\operatorname{resp} .\{t\} \times\left[\sigma^{-}(t), \sigma^{+}(t)\right]\right)$ to the point $\left(t, \gamma^{+}(t)\right)$, hence it is not injective. More generally, a semicartesian map could be possibly not injective even if $\gamma^{-}(t) \neq \gamma^{+}(t)$ for every $t \in(a, b)$.

As a consequence of Lemma 2.8, we can introduce the following quantities.

Definition 2.10 (Minimal values $m$ ) Let $\Gamma=\Gamma^{-} \cup \Gamma^{-}$be union of two Lipschitz graphs on $[a, b]$. We define

$$
m\left(\mathrm{R} ; \Gamma^{-}, \Gamma^{+}\right):=\inf _{\Phi \in \operatorname{semicart}\left(\mathrm{R} ; \Gamma^{-}, \Gamma^{+}\right)} \int_{\mathrm{R}}\left|\partial_{t} \Phi \wedge \partial_{s} \Phi\right| \mathrm{d} t \mathrm{~d} s .
$$

If furthermore $\Gamma$ is closed we define

$$
m\left(D ; \Gamma^{-}, \Gamma^{+}\right):=\inf _{\Phi \in \operatorname{semicart}\left(D ; \Gamma^{-}, \Gamma^{+}\right)} \int_{D}\left|\partial_{t} \Phi \wedge \partial_{s} \Phi\right| \mathrm{d} t \mathrm{~d} s .
$$


It is worthwhile to observe that (2.4) may become a partially free boundary problem, on the planes $\{t=a\}$ and $\{t=b\}$.

Trivially, if $\Gamma$ is closed, then

$$
m\left(\mathrm{R} ; \Gamma^{-}, \Gamma^{+}\right) \leq m\left(D ; \Gamma^{-}, \Gamma^{+}\right) .
$$

Indeed, supposing without loss of generality that $\left|\sigma^{ \pm}\right|<1$, we can find, for any $\Psi \in$ $\operatorname{semicart}\left(D ; \Gamma^{-}, \Gamma^{+}\right)$, a map $\Phi \in \operatorname{semicart}\left(\mathrm{R} ; \Gamma^{-}, \Gamma^{+}\right)$having the same area, defined as

$$
\Phi(t, s):= \begin{cases}\Psi(t, s) & \text { if }(t, s) \in D, \\ \gamma^{+}(t) & \text { if }(t, s) \in \mathrm{R} \backslash D, s>0, \\ \gamma^{-}(t) & \text { if }(t, s) \in \mathrm{R} \backslash D, s<0 .\end{cases}
$$

Remark 2.11 (Semicartesian parametrizations and Plateau's problem) The problem of the existence of a minimum in (2.4) and (2.5) seems to be open and requires further investigation. If $\Gamma=\Gamma^{-} \cup \Gamma^{+}$is a closed simple curve, it is natural to compare $m\left(D ; \Gamma^{-}, \Gamma^{+}\right)$with the area $a(\Gamma)$ of a solution of the classical Plateau's problem for $\Gamma$, that is an area-minimizing immersion of the disk, that maps the boundary of the disk onto $\Gamma$ monotonically, see for instance [13] and [8]. It is possible ${ }^{5}$ to see that

$$
m\left(D ; \Gamma^{-}, \Gamma^{+}\right) \geq a(\Gamma) .
$$

It is plausible that (2.7) holds with equal sign, and that $m\left(D ; \Gamma^{-}, \Gamma^{+}\right)$is actually a minimum. To substantiate these assertions, we recall that in [4] it is proven that solutions of the classical Plateau's problem admit a semicartesian parametrization if $\Gamma$ is an analytic curve with further non-degeneracy properties at $\left(a, \gamma^{ \pm}(a)\right)$ and $\left(b, \gamma^{ \pm}(b)\right)$ (a case that does not fit in our setting). On the other hand, for what concerns the semicartesian maps defined on the rectangle $\mathrm{R}$, even assuming that $\Gamma$ is a closed simple curve, the existence of an area-minimizing semicartesian parametrization in semicart $\left(\mathrm{R} ; \Gamma^{-}, \Gamma^{+}\right)$does not follow from the existence of a solution for the Plateau's problem. Indeed since the class of surfaces parametrized by maps in semicart $\left(\mathrm{R} ; \Gamma^{-}, \Gamma^{+}\right)$strictly contains (due to the free boundary on the planes $\{t=a\}$ and $\{t=b\})$ the ones parametrized by maps in $\operatorname{semicart}\left(D ; \Gamma^{-}, \Gamma^{+}\right)$, we could expect that in general it contains also the class of surfaces considered in the classical setting. Moreover, we shall prove that possibly $a(\Gamma)>m\left(\mathrm{R} ; \Gamma^{-}, \Gamma^{+}\right)$: in Example 2.12 we build a semicartesian parametrization whose image is not in the class of surfaces considered for the classical Plateau's problem; in Example 2.13 we exhibit $\gamma^{ \pm} \in \operatorname{Lip}\left([a, b] ; \mathbb{R}^{2}\right)$ such that the union of their graphs is a Jordan curve for which the semicartesian parametrization built in Example 2.12 has as area which is less than $a(\Gamma)$.

The next example is also strictly related to the construction made in Proposition 7.1.

Example 2.12 (Partially free boundary on $\{t=b\}$ ) Let $\gamma^{ \pm} \in \operatorname{Lip}\left([a, b] ; \mathbb{R}^{2}\right)$ and suppose that $\gamma^{-}(a)=\gamma^{+}(a)$; let us denote by $\mathrm{C}$ the (connected) set $\gamma^{-}([a, b]) \cup \gamma^{+}([a, b]) \subset \mathbb{R}_{(\xi, \eta)}^{2}$. In Fig. 1 we draw a case when $\gamma^{+}$is not injective.

We want to define the map $\Phi \in \operatorname{semicart}\left(\mathrm{R} ; \Gamma^{-}, \Gamma^{+}\right)$which, for every $t \in(a, b)$, maps the segment $\{t\} \times[-1,1] \subset \mathrm{R}$ onto the portion of $\{t\} \times \mathrm{C}$ bounded by the points $\left(t, \gamma^{-}(t)\right)$ and $\left(t, \gamma^{+}(t)\right)$ and containing $\left(t, \gamma^{-}(a)\right)$.

5 For instance, as a consequence of the Riemann mapping theorem. 


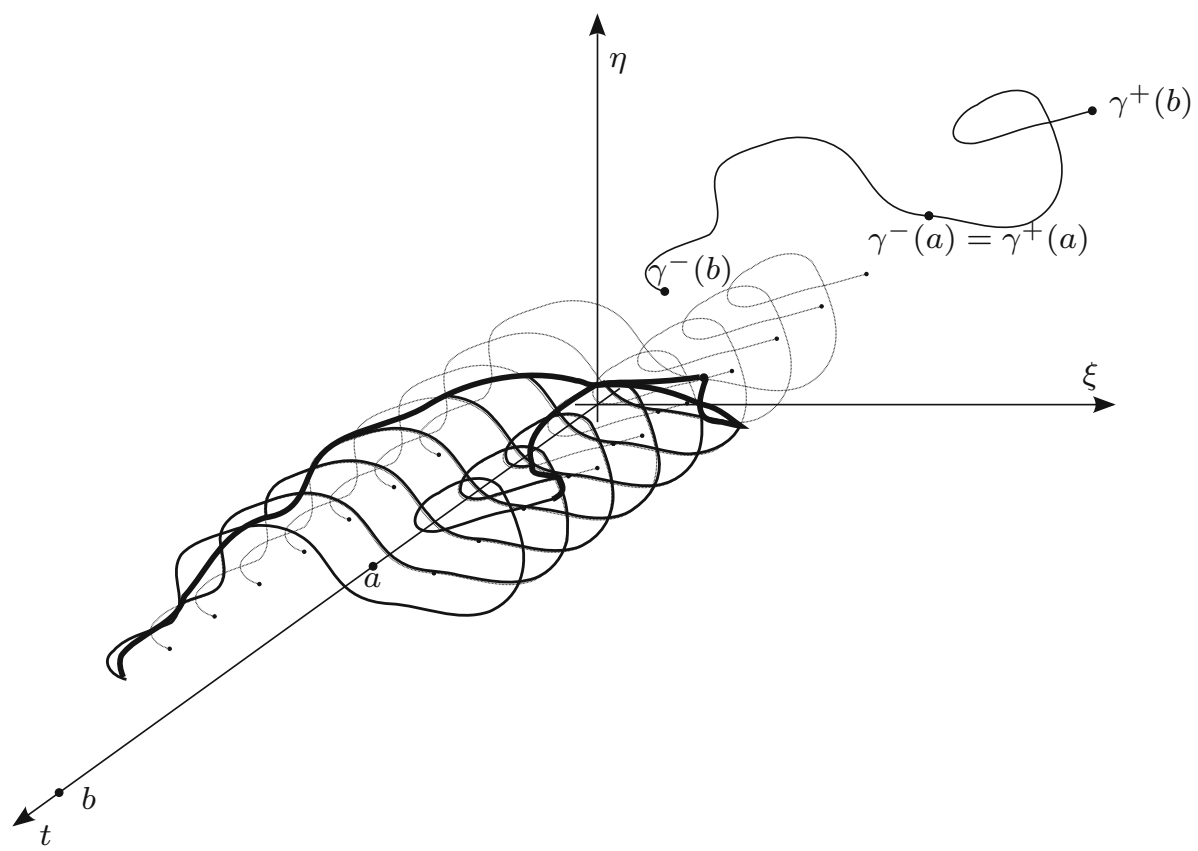

Fig. 1 Example 2.12. In the plane $\{0\} \times \mathbb{R}_{(\xi, \eta)}^{2}$ we represent the curve C; $\mathrm{C}$ is the projection of the curves $\Gamma^{ \pm}$ (in bold) on the plane $\{0\} \times \mathbb{R}_{(\xi, \eta)}^{2}$. In light gray we draw the copies of $\mathrm{C}$ in the planes $\{t\} \times \mathbb{R}_{(\xi, \eta)}^{2}, t \in[a, b]$. The surface $\Phi(\mathrm{R})$ is the union of all portions of $\{t\} \times \mathrm{C}$ bounded by $\left(t, \gamma^{-}(t)\right)$ and $\left(t, \gamma^{+}(t)\right)$, when $t$ varies in $[a, b]$

If for convenience we parametrize $C$ by a curve $\gamma \in \operatorname{Lip}\left([-1,1] ; \mathbb{R}^{2}\right)$, defined by

$$
\gamma(\lambda):= \begin{cases}\gamma^{-}(-(b-a) \lambda+a) & \text { if } \lambda \in[-1,0], \\ \gamma^{+}((b-a) \lambda+a) & \text { if } \lambda \in(0,1],\end{cases}
$$

so that $\gamma(-1)=\gamma^{-}(b), \gamma(0)=\gamma^{-}(a)=\gamma^{+}(a)$ and $\gamma(1)=\gamma^{+}(b)$, then $\Phi(\{t\} \times[-1,1])$ must be equal to $\left\{(t, \gamma(\lambda)): \lambda \in\left[-\frac{t-a}{b-a}, \frac{t-a}{b-a}\right]\right\}$. Thus we can define $\Phi \in \operatorname{semicart}\left(\mathrm{R} ; \Gamma^{-}, \Gamma^{+}\right)$as

$$
\Phi(t, s):=\left(t, \gamma\left(\frac{t-a}{b-a} s\right)\right), \quad(t, s) \in \mathrm{R} .
$$

We observe that, if $\Gamma:=\operatorname{graph}\left(\gamma^{-}\right) \cup \operatorname{graph}\left(\gamma^{+}\right)$is a closed simple curve, the surface $\Phi(\mathrm{R})$ is not the image of an immersion of the disk mapping the boundary of the disk monotonically onto $\Gamma$, because $\Phi(\partial R)=\Gamma \cup(\{b\} \times \mathrm{C})$. Moreover,

$$
m\left(\mathrm{R} ; \Gamma^{-}, \Gamma^{+}\right) \leq \int_{\mathrm{R}}\left|\partial_{t} \Phi \wedge \partial_{s} \Phi\right| \mathrm{d} t \mathrm{~d} s \leq(b-a) \int_{a}^{b}\left(\left|\dot{\gamma}^{-}\right|+\left|\dot{\gamma}^{+}\right|\right) \mathrm{d} t .
$$

Note that, if $\gamma^{ \pm}$are injective, we have that $\Phi(\mathrm{R})$ lies on the lateral part of the surface of the cylinder $(a, b) \times \mathrm{C}$.

We now exhibit maps $\gamma^{ \pm} \in \operatorname{Lip}\left([a, b] ; \mathbb{R}^{2}\right)$ so that $\Gamma:=\operatorname{graph}\left(\gamma^{-}\right) \cup \operatorname{graph}\left(\gamma^{+}\right)$is a closed simple curve and $m\left(\mathrm{R} ; \Gamma^{-}, \Gamma^{+}\right)<a(\Gamma)$. 

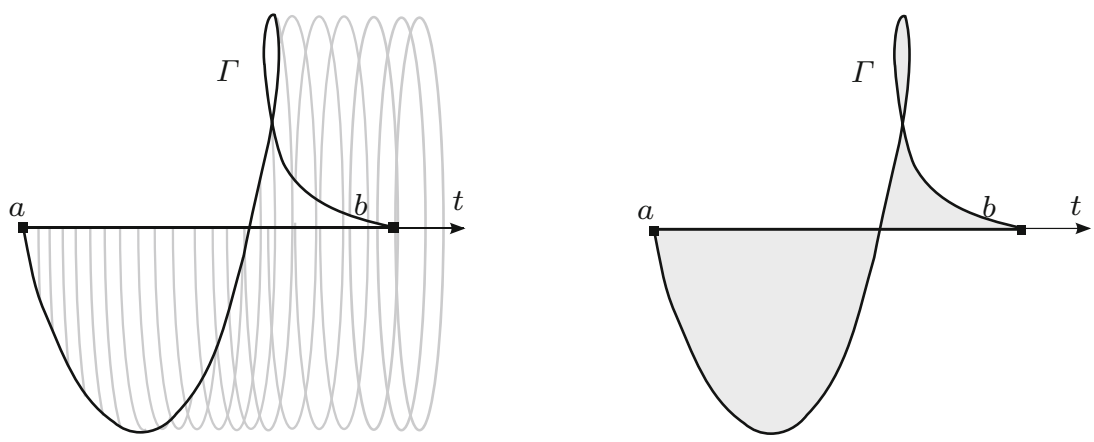

Fig. 2 The curve $\Gamma$ defined in Example 2.13. The left picture is the image of the semicartesian parametrization $(\mathrm{R}, \Phi)$ spanning $\Gamma$ built as in Example 2.12; we notice that it lies on the lateral surface of the cylinder of base the disk of radius $\rho$ and height $b-a$. The right picture represents the image of an embedding of the disk mapping the boundary of the disk onto $\Gamma$ : the area of such a surface is greater than or equal to the area of its orthogonal projection on a plane orthogonal to the $t$ axis, that is a disk of radius $\rho$

Example $2.13\left(m\left(\mathrm{R} ; \Gamma^{-}, \Gamma^{+}\right)<a(\Gamma)\right)$ Let $\rho$ be a positive real number with

$$
\rho>2(b-a) \text {. }
$$

Let us define the maps $\gamma^{ \pm} \in \operatorname{Lip}\left([a, b] ; \mathbb{R}^{2}\right)$ as follows: if $t \in[a, b]$,

$$
\begin{aligned}
& \gamma^{-}(t):=(1,0), \\
& \gamma^{+}(t):=\rho(\cos (\theta(t)), \sin (\theta(t)))+(1-\rho, 0),
\end{aligned}
$$

where $\theta:[a, b] \rightarrow[0,2 \pi]$ is given by

$$
\theta(t):=\frac{2 \pi(t-a)}{b-a}, \quad t \in[a, b]
$$

see the second picture of Fig. 2. Then $\Gamma:=\operatorname{graph}\left(\gamma^{-}\right) \cup \operatorname{graph}\left(\gamma^{+}\right)$is a Lipschitz closed simple curve. Moreover, any disk-type surface spanning $\Gamma$ has area greater than or equal to the area $\pi \rho^{2}$ of its orthogonal projection (a disk of radius $\rho$ ) on the coordinate plane $\mathbb{R}_{(\xi, \eta)}^{2}$, hence

$$
a(\Gamma) \geq \pi \rho^{2} .
$$

On the other hand, the image $\Phi(\mathrm{R})$ of the semicartesian parametrization $(\mathrm{R}, \Phi)$ defined in (2.8) has area strictly less than $2 \pi \rho(b-a)$ (see the first picture in Fig. 2). From our choice (2.9), it then follows

$$
m\left(\mathrm{R} ; \Gamma^{-}, \Gamma^{+}\right)<a(\Gamma) .
$$

We conclude this section proving that fixing $\mathrm{R}$ and $D$ as in Definition 2.5 is not restrictive.

Lemma 2.14 (Choice of domain) Let $\Gamma=\Gamma^{-} \cup \Gamma^{+}$be union of two Lipschitz graphs on $[a, b], \Gamma^{ \pm}=\operatorname{graph}\left(\gamma^{ \pm}\right)$. Let $O_{1}=\left[\left[\sigma_{1}^{-}, \sigma_{1}^{+}\right]\right]$be such that $\sigma_{1}^{-}(a)<\sigma_{1}^{+}(a)$ and $\sigma_{1}^{-}(b)<\sigma_{1}^{+}(b)$. If $\left(O_{1}, \Psi\right)$ is a semicartesian parametrization spanning $\Gamma$ such that $\Psi \in$ $H^{1}\left(O_{1} ; \mathbb{R}^{3}\right)$, then there exists a map $\Phi \in \operatorname{semicart}\left(\mathrm{R} ; \Gamma^{-}, \Gamma^{+}\right)$such that

$$
\int_{\mathrm{R}}\left|\partial_{t} \Phi \wedge \partial_{s} \Phi\right| d t d s=\int_{O_{1}}\left|\partial_{t} \Psi \wedge \partial_{s} \Psi\right| d t d s
$$


If moreover $\Gamma$ is closed, $\mathrm{O}_{2}=\left[\left[\sigma_{2}^{+}, \sigma_{2}^{+}\right]\right]$is such that $\sigma_{2}^{-}(a)=\sigma_{2}^{+}(a)$ and $\sigma_{2}^{-}(b)=\sigma_{2}^{+}(b)$, and $\left(\mathrm{O}_{2}, \chi\right)$ is a semicartesian parametrization spanning $\Gamma$ such that $\chi \in H^{1}\left(O_{2} ; \mathbb{R}^{3}\right)$, then there exists a map $\Phi \in \operatorname{semicart}\left(D ; \Gamma^{-}, \Gamma^{+}\right)$such that

$$
\int_{D}\left|\partial_{t} \Phi \wedge \partial_{s} \Phi\right| d t d s=\int_{O_{2}}\left|\partial_{t} \chi \wedge \partial_{s} \chi\right| d t d s .
$$

Proof Let us define the map $T_{1}: \mathrm{R} \rightarrow O_{1}$ as

$$
T_{1}(t, s):=\left(t, \frac{1-s}{2} \sigma_{1}^{-}(t)+\frac{1+s}{2} \sigma_{1}^{+}(t)\right) .
$$

Since $\sigma_{1}^{ \pm} \in \operatorname{Lip}([a, b])$, we have that $T \in \operatorname{Lip}\left(\mathrm{R} ; O_{1}\right)$ and thus the map $\Phi:=\Psi \circ T_{1}$ belongs to semicart(R; $\left.\Gamma^{-}, \Gamma^{+}\right)$; moreover $T_{1}$ is injective and thus (2.12) holds.

Let us suppose that $\Gamma$ is closed. Recall that $D=\left[\left[\sigma^{-}, \sigma^{+}\right]\right]$. We define the map $T_{2}$ : $D \rightarrow \mathrm{O}_{2}$ as

$$
T_{2}(t, s):=\left(t, \frac{\sigma^{+}(t)-s}{\sigma^{+}(t)-\sigma^{-}(t)} \sigma_{2}^{-}(t)+\frac{s-\sigma^{-}(t)}{\sigma^{+}(t)-\sigma^{-}(t)} \sigma_{2}^{+}(t)\right) .
$$

One can show that $T_{2} \in \operatorname{Lip}\left(D ; O_{2}\right)$ with a computation similar to the one in Lemma 2.8, and thus the map $\Phi:=\chi \circ T_{2}$ belongs to semicart $\left(D ; \Gamma^{-}, \Gamma^{+}\right)$. The injectivity of $T_{2}$ implies (2.13).

\subsection{Maps from a planar domain to the plane and jumping on a curve}

From now on we set

$$
\mathrm{R}^{-}:=(a, b) \times(-1,0), \quad \mathrm{R}^{+}:=(a, b) \times(0,1) .
$$

Let $\Omega \subset \mathbb{R}_{(x, y)}^{2}$ be a bounded open connected set. Let $\mathbf{u}: \Omega \rightarrow \mathbb{R}_{(\xi, \eta)}^{2}$ be a map belonging to $\operatorname{BV}\left(\Omega ; \mathbb{R}^{2}\right) \cap W^{1, \infty}\left(\Omega \backslash J_{\mathbf{u}} ; \mathbb{R}^{2}\right)$, where $J_{\mathbf{u}} \subset \Omega$ is a $\mathcal{C}^{2}$ simple curve parametrized by an arc-length parametrization $\alpha:(a, b) \subset \mathbb{R}_{t} \rightarrow \mathbb{R}_{(x, y)}^{2}$. Two cases are possible (remember our convention on the set $J_{\mathbf{u}}$ in the Introduction): either $J_{\mathbf{u}} \subset \subset \Omega$ or $J_{\mathbf{u}} \cap \partial \Omega \neq \emptyset$.

We denote by $\mathbf{u}^{ \pm}$the two Lipschitz traces on the two sides of the jump, and we define $\gamma^{ \pm}[\mathbf{u}] \in \operatorname{Lip}\left((a, b) ; \mathbb{R}^{2}\right)$ as

$$
\gamma^{ \pm}[\mathbf{u}](t):=\mathbf{u}^{ \pm}(\alpha(t))
$$

In accordance with our previous notation, we denote with $\Gamma^{ \pm}[\mathbf{u}] \subset \mathbb{R}^{3}=\mathbb{R}_{t} \times \mathbb{R}_{(\xi, \eta)}^{2}$ the graph of $\gamma^{ \pm}[\mathbf{u}]$. When there is no ambiguity, we shall write $\gamma^{ \pm}$and $\Gamma^{ \pm}$in place of $\gamma^{ \pm}[\mathbf{u}]$ and $\Gamma^{ \pm}[\mathbf{u}]$, respectively.

In this paper, we will deal with pairs $(\Omega, \mathbf{u})$ satisfying one of the two conditions specified in Definitions 2.15 and 2.16. In both the two conditions, the jump $J_{\mathbf{u}}$ is a horizontal segment; this assumption allows to identify the plane $\mathbb{R}_{(x, y)}^{2}$ (containing the domain $\Omega$ of $\mathbf{u}$ ) with the space of the parameters $\mathbb{R}_{(t, s)}^{2}$, thus simplifying the presentation. ${ }^{6}$

\footnotetext{
6 When $J_{\mathbf{u}}$ is a simple curve of class $\mathcal{C}^{2}$, this identification cannot be made; however there exist $\delta>0$ and an open set $N \supset J_{\mathbf{u}}$ such that $N=\Lambda((a, b) \times(-\delta, \delta))$, with $(a, b) \times(-\delta, \delta) \subset \mathbb{R}_{(t, s)}$ and $\Lambda$ is the $\mathcal{C}^{1}$ diffeomorphism defined by $\Lambda(t, s):=\alpha(t)+s \dot{\alpha}(t)^{\perp}$ for $(t, s) \in \mathrm{R}_{\delta}:=(a, b) \times(-\delta, \delta)$, where $\mathbf{v}^{\perp}:=\left(-v_{2}, v_{1}\right)$. Thus, with some technicalities, we expect to be possible to extend our results also to this case. In [4] the upper bound to $\overline{\mathcal{A}}(\mathbf{u}, \Omega)$ is provided for a map jumping on a $\mathcal{C}^{2}$ smooth curve compactly contained in $\Omega$.
} 
Definition 2.15 (Condition I) We say that $\Omega$ and $\mathbf{u} \in \mathrm{BV}\left(\Omega ; \mathbb{R}^{2}\right)$ satisfy condition I if $\Omega=\mathrm{R}, J_{\mathbf{u}}=(a, b) \times\{0\}$, and $\mathbf{u} \in \operatorname{Lip}\left(\mathrm{R}^{-} ; \mathbb{R}^{2}\right) \cap \operatorname{Lip}\left(\mathrm{R}^{+} ; \mathbb{R}^{2}\right)$.

Definition 2.16 (Condition II) We say that $\Omega$ and $\mathbf{u} \in \mathrm{BV}\left(\Omega ; \mathbb{R}^{2}\right)$ satisfy condition II if $J_{\mathbf{u}}:=[a, b] \times\{0\} \subset \subset \Omega, \mathbf{u} \in W^{1, \infty}\left(\Omega \backslash J_{\mathbf{u}} ; \mathbb{R}^{2}\right)$, and there exist the pointwise limits (still denoted by $\mathbf{u}^{ \pm}$) of $\mathbf{u}$ at all points of $J_{\mathbf{u}}$.

\section{Condition I: upper bound}

The next proposition provides an upper bound for $\overline{\mathcal{A}}^{\infty}(\mathbf{u}, \Omega)$ (and hence for $\overline{\mathcal{A}}(\mathbf{u}, \Omega)$ ), when $\Omega$ and $\mathbf{u}$ satisfy condition I, proving one of the two inequalities (i.e., (3.2)) of Theorem 1.3. We shall suitably modify the construction made in [4] in a different context.

Proposition 3.1 (Upper bound, I) Let $\Omega$ and $\mathbf{u}$ satisfy condition I. Then there exists a sequence $\left(\mathbf{u}_{h}\right) \subset H^{1}\left(\mathrm{R} ; \mathbb{R}^{2}\right)$ converging to $\mathbf{u}$ in $L^{1}\left(\mathrm{R} ; \mathbb{R}^{2}\right)$ and uniformly out of $J_{\mathbf{u}}$ such that

$$
\liminf _{h \rightarrow+\infty} \mathcal{A}\left(\mathbf{u}_{h}, \mathrm{R}\right)=\int_{\mathrm{R}}|\mathcal{M}(\nabla \mathbf{u})| d t d s+m\left(\mathrm{R} ; \Gamma^{-}[\mathbf{u}], \Gamma^{+}[\mathbf{u}]\right) .
$$

Hence

$$
\overline{\mathcal{A}}_{s}^{\infty}(\mathbf{u}, \mathrm{R}) \leq m\left(\mathrm{R} ; \Gamma^{-}[\mathbf{u}], \Gamma^{+}[\mathbf{u}]\right) .
$$

Proof Let $\left(\Phi_{h}\right) \subset \operatorname{semicart}\left(\mathrm{R} ; \Gamma^{-}, \Gamma^{+}\right)$be a minimizing sequence for (1.3), that is

$$
\int_{\mathrm{R}}\left|\partial_{t} \Phi_{h} \wedge \partial_{s} \Phi_{h}\right| d t d s \rightarrow m\left(\mathrm{R} ; \Gamma^{-}, \Gamma^{+}\right) \text {as } h \rightarrow+\infty
$$

and write $\Phi_{h}(t, s)=\left(t, \phi_{h}(t, s)\right)$ with $\phi_{h} \in H^{1}\left(\mathrm{R} ; \mathbb{R}^{2}\right)$. For any $\varepsilon \in(0,1)$ set $\mathrm{R}_{\varepsilon}:=$ $(a, b) \times(-\varepsilon, \varepsilon)$, and define the map $\mathbf{u}_{h, \varepsilon} \in H^{1}\left(\mathrm{R} ; \mathbb{R}^{2}\right)$ as

$$
\mathbf{u}_{h, \varepsilon}(t, s):= \begin{cases}\mathbf{u}(t, s) & \text { if }(t, s) \in \mathrm{R} \backslash \mathrm{R}_{2 \varepsilon}, \\ \mathbf{u}(t, 2(s-\varepsilon)) & \text { if }(t, s) \in(a, b) \times(\varepsilon, 2 \varepsilon), \\ \mathbf{u}(t, 2(s+\varepsilon)) & \text { if }(t, s) \in(a, b) \times(-2 \varepsilon,-\varepsilon), \\ \phi_{h}(t, s / \varepsilon) & \text { if }(t, s) \in \overline{\mathrm{R}_{\varepsilon}} .\end{cases}
$$

With a computation similar to the one in [4], we get

$$
\liminf _{\varepsilon \rightarrow 0^{+}} \mathcal{A}\left(\mathbf{u}_{h, \varepsilon}, \mathrm{R}\right)=\int_{\mathrm{R}}|\mathcal{M}(\nabla \mathbf{u})| \mathrm{d} t \mathrm{~d} s+\int_{\mathrm{R}}\left|\partial_{t} \Phi_{h} \wedge \partial_{s} \Phi_{h}\right| \mathrm{d} t \mathrm{~d} s .
$$

Indeed $\mathcal{A}\left(\mathbf{u}_{h, \varepsilon},(a, b) \times(\varepsilon, 2 \varepsilon)\right)$ and $\mathcal{A}\left(\mathbf{u}_{h, \varepsilon},(a, b) \times(-2 \varepsilon,-\varepsilon)\right)$ are negligible as $\varepsilon \rightarrow 0^{+}$, as a consequence of the hypothesis $\mathbf{u} \in \operatorname{Lip}\left(\mathrm{R}^{+} ; \mathbb{R}^{2}\right) \cap \operatorname{Lip}\left(\mathrm{R}^{-} ; \mathbb{R}^{2}\right)$. Moreover, a direct computation gives:

$$
\begin{aligned}
\mathcal{A}\left(\mathbf{u}_{h, \varepsilon}, \mathrm{R}_{\varepsilon}\right)= & \int_{a}^{b} \int_{-\varepsilon}^{\varepsilon} \sqrt{1+\left|\partial_{t} \phi_{h}\left(t, \frac{s}{\varepsilon}\right)\right|^{2}+\frac{1}{\varepsilon^{2}}\left|\partial_{s} \phi_{h}\left(t, \frac{s}{\varepsilon}\right)\right|^{2}+\frac{1}{\varepsilon^{2}}\left(\operatorname{det} \nabla \phi_{h}\left(t, \frac{s}{\varepsilon}\right)\right)^{2}} \mathrm{~d} s \mathrm{~d} t \\
= & \int_{a}^{b} \int_{-1}^{1} \sqrt{\left|\partial_{s} \phi_{h}(t, s)\right|^{2}+\left(\operatorname{det} \nabla \phi_{h}(t, s)\right)^{2}+\mathcal{O}\left(\varepsilon^{2}\right)} \mathrm{d} s \mathrm{~d} t \\
& \stackrel{\varepsilon \rightarrow 0^{+}}{\longrightarrow} \int_{\mathrm{R}}\left|\partial_{t} \Phi_{h} \wedge \partial_{s} \Phi_{h}\right| \mathrm{d} t \mathrm{~d} s .
\end{aligned}
$$


By a diagonalization process, and using (3.3), we can choose a sequence $\left(\mathbf{u}_{h}\right):=\left(\mathbf{u}_{h, \varepsilon_{h}}\right)$ such that

$$
\lim _{h \rightarrow+\infty} \mathcal{A}\left(\mathbf{u}_{h}, \mathrm{R}\right)=\int_{\mathrm{R}}|\mathcal{M}(\nabla \mathbf{u})| \mathrm{d} t \mathrm{~d} s+m\left(\mathrm{R} ; \Gamma^{-}, \Gamma^{+}\right),
$$

which implies (3.1). ${ }^{7}$

\section{Condition I: lower bound}

The main result of this section is the following inequality that, coupled with Proposition 3.1, concludes the proof of Theorem 1.3.

Theorem 4.1 (Lower bound, I) Let $\Omega$ and $\mathbf{u}$ satisfy condition $\mathrm{I}$. Let $\left(\mathbf{u}_{h}\right) \subset \operatorname{Lip}\left(\mathrm{R} ; \mathbb{R}^{2}\right)$ be a sequence converging to $\mathbf{u}$ in $L^{1}\left(\mathrm{R} ; \mathbb{R}^{2}\right)$ and uniformly out of $J_{\mathbf{u}}$. Then

$$
\liminf _{h \rightarrow+\infty} \mathcal{A}\left(\mathbf{u}_{h}, \mathrm{R}\right) \geq \int_{\mathrm{R}}|\mathcal{M}(\nabla \mathbf{u})| d t d s+m\left(\mathrm{R} ; \Gamma^{-}[\mathbf{u}], \Gamma^{+}[\mathbf{u}]\right) .
$$

Hence

$$
\overline{\mathcal{A}}_{s}^{\infty}(\mathbf{u}, \mathrm{R}) \geq m\left(\mathrm{R} ; \Gamma^{-}[\mathbf{u}], \Gamma^{+}[\mathbf{u}]\right) .
$$

The proof of Theorem 4.1 will be achieved in two steps: the first step gives the result if the sequence $\mathbf{u}_{h}$ coincides with $\mathbf{u}$ far enough from $J_{\mathbf{u}}$.

Theorem 4.2 Let $\Omega$ and $\mathbf{u}$ satisfy condition I. If $\left(\mathbf{u}_{h}\right) \subset \operatorname{Lip}\left(\mathrm{R} ; \mathbb{R}^{2}\right)$ converges to $\mathbf{u}$ in $L^{1}\left(\mathrm{R} ; \mathbb{R}^{2}\right)$ and

$$
\mathbf{u}_{h}=\mathbf{u} \quad \text { in } \mathrm{R} \backslash N_{h},
$$

for some decreasing sequence $\left(N_{h}\right)$ of neighborhoods of $J_{\mathbf{u}}$ such that $\bigcap_{h \in \mathbb{N}} N_{h}=J_{\mathbf{u}}$, then

$$
\liminf _{h \rightarrow+\infty} \mathcal{A}\left(\mathbf{u}_{h}, \mathrm{R}\right) \geq \int_{\mathrm{R}}|\mathcal{M}(\nabla \mathbf{u})| d t d s+m\left(\mathrm{R} ; \Gamma^{-}[\mathbf{u}], \Gamma^{+}[\mathbf{u}]\right)
$$

The second step shows that, given any sequence $\left(\mathbf{u}_{h}\right)$ satisfying the hypotheses of Theorem 4.1, we can build a sequence ( $\left.\mathbf{v}_{h}\right)$ satisfying the hypotheses of Theorem 4.2 and whose area is, in the limit, not larger than the area of $\left(\mathbf{u}_{h}\right)$.

Theorem 4.3 Let $\Omega$ and $\mathbf{u}$ satisfy condition $\mathrm{I}$. Let $\left(\mathbf{u}_{h}\right) \subset \mathrm{Lip}\left(\mathrm{R} ; \mathbb{R}^{2}\right)$ be a sequence converging to $\mathbf{u}$ in $L^{1}\left(\mathrm{R} ; \mathbb{R}^{2}\right)$ and uniformly out of $J_{\mathbf{u}}$. Then there exists a sequence $\left(\mathbf{v}_{h}\right) \subset \operatorname{Lip}\left(\mathrm{R} ; \mathbb{R}^{2}\right)$ satisfying the hypotheses of Theorem 4.2 and such that

$$
\liminf _{h \rightarrow+\infty} \mathcal{A}\left(\mathbf{u}_{h}, \mathrm{R}\right) \geq \liminf _{h \rightarrow+\infty} \mathcal{A}\left(\mathbf{v}_{h}, \mathrm{R}\right) .
$$

\subsection{Proof of Theorem 4.2}

We need some preliminary lemmas.

\footnotetext{
$\overline{7}$ Indeed (see [4, Appendix 3]) $\overline{\mathcal{A}}(\cdot, \mathrm{R})$ can be obtained by relaxing in $L^{1}$ the functional $\mathbf{v} \rightarrow$ $\int_{\mathrm{R}}|\mathcal{M}(\nabla \mathbf{v})| d t d s$ from $H^{1}\left(\mathrm{R} ; \mathbb{R}^{3}\right)$. Similar results hold for $\overline{\mathcal{A}}^{\infty}(\cdot, \mathrm{R})$, with the corresponding convergence.
} 
Lemma 4.4 (Lower bound of $m$ via interpolation) Let $\alpha, \beta \in \operatorname{Lip}\left([a, b] ; \mathbb{R}^{2}\right)$, and set $\Gamma_{\alpha}:=$ $\operatorname{graph}(\alpha)$ and $\Gamma_{\beta}:=\operatorname{graph}(\beta)$. Then there exists a constant $C>0$ independent of $\alpha$ and $\beta$, such that

$$
m\left(\mathrm{R} ; \Gamma_{\alpha}, \Gamma_{\beta}\right) \leq C\|\alpha-\beta\|_{L^{1}\left((a, b) ; \mathbb{R}^{2}\right)}\left(1+\max \left[\|\dot{\alpha}\|_{L^{\infty}\left((a, b) ; \mathbb{R}^{2}\right)},\|\dot{\beta}\|_{L^{\infty}\left((a, b) ; \mathbb{R}^{2}\right)}\right]\right) .
$$

Proof Let us define the map $\ell \in W^{1, \infty}\left(\mathrm{R} ; \mathbb{R}^{2}\right)$ interpolating $\alpha$ and $\beta$, as in Lemma 2.8, that is

$$
\ell(t, s):=\frac{1-s}{2} \alpha(t)+\frac{1+s}{2} \beta(t), \quad(t, s) \in \mathrm{R} .
$$

Setting $\Phi_{\ell}(t, s):=(t, \ell(t, s))$, we get $\partial_{t} \Phi_{\ell}(t, s)=\left(1, \frac{1-s}{2} \dot{\alpha}(t)+\frac{1+s}{2} \dot{\beta}(t)\right)$ and $\partial_{s} \Phi_{\ell}(t, s)=\left(0, \frac{\beta(t)-\alpha(t)}{2}\right)$. Thus

$$
\left|\partial_{t} \Phi_{\ell} \wedge \partial_{s} \Phi_{\ell}\right|=\frac{1}{2} \sqrt{|\alpha-\beta|^{2}+\left[\left(\frac{1-s}{2} \dot{\alpha}+\frac{1+s}{2} \dot{\beta}\right) \cdot(\alpha-\beta)^{\perp}\right]^{2}},
$$

where, for $z=\left(z_{1}, z_{2}\right) \in \mathbb{R}^{2}$, we set $z^{\perp}:=\left(-z_{2}, z_{1}\right)$. Hence

$$
\begin{aligned}
& \int_{\mathrm{R}}\left|\partial_{t} \Phi_{\ell} \wedge \partial_{s} \Phi_{\ell}\right| d t d s \\
& \quad \leq C\|\alpha-\beta\| \|_{L^{1}\left((a, b) ; \mathbb{R}^{2}\right)}\left(1+\max \left[\|\dot{\alpha}\|_{L^{\infty}\left((a, b) ; \mathbb{R}^{2}\right)},\|\dot{\beta}\|_{L^{\infty}\left((a, b) ; \mathbb{R}^{2}\right)}\right]\right),
\end{aligned}
$$

and, since $\Phi_{\ell} \in \operatorname{semicart}\left(\mathrm{R} ; \Gamma_{\alpha}, \Gamma_{\beta}\right)$, also (4.3) follows.

The computations in Lemma 4.4 allow to prove a semicontinuity result for $m\left(\mathrm{R} ; \Gamma_{h}^{-}, \Gamma_{h}^{+}\right)$.

Lemma 4.5 (Lower semicontinuity of $m(\mathrm{R} ; \cdot, \cdot))$ Let $\left(\gamma_{h}^{ \pm}\right) \subset \operatorname{Lip}\left([a, b] ; \mathbb{R}^{2}\right)$ and $\gamma^{ \pm} \in$ $\operatorname{Lip}\left([a, b] ; \mathbb{R}^{2}\right)$ be such that:

- there exists $C_{1}>0$ such that $\left\|\dot{\gamma}_{h}^{ \pm}\right\|_{L^{\infty}\left((a, b) ; \mathbb{R}^{2}\right)} \leq C_{1}$ for any $h \in \mathbb{N}$,

$-\gamma_{h}^{ \pm} \rightarrow \gamma^{ \pm}$in $L^{1}\left((a, b) ; \mathbb{R}^{2}\right)$ as $h \rightarrow+\infty$.

Then, setting $\Gamma_{h}^{ \pm}:=\operatorname{graph}\left(\gamma_{h}^{ \pm}\right)$and $\Gamma^{ \pm}:=\operatorname{graph}\left(\gamma^{ \pm}\right)$, we have

$$
m\left(\mathrm{R} ; \Gamma^{-}, \Gamma^{+}\right) \leq \liminf _{h \rightarrow+\infty} m\left(\mathrm{R} ; \Gamma_{h}^{-}, \Gamma_{h}^{+}\right) .
$$

Proof For any $h \in \mathbb{N}$, let $\left(\Phi_{k}^{h}\right) \subset \operatorname{semicart}\left(\mathrm{R} ; \Gamma_{h}^{-}, \Gamma_{h}^{+}\right)$be such that

$$
\lim _{k \rightarrow+\infty} \int_{\mathrm{R}}\left|\partial_{t} \Phi_{k}^{h} \wedge \partial_{s} \Phi_{k}^{h}\right| d t d s=m\left(\mathrm{R} ; \Gamma_{h}^{-}, \Gamma_{h}^{+}\right) .
$$

Let us denote by $\ell_{h}^{+}, \ell_{h}^{-}: \mathrm{R} \rightarrow \mathbb{R}^{2}$ the linear interpolating maps, such that, for any $t \in[a, b]$,

$$
\begin{array}{ll}
\ell_{h}^{+}(t,-1)=\gamma_{h}^{+}(t), & \ell_{h}^{+}(t, 1)=\gamma^{+}(t), \\
\ell_{h}^{-}(t,-1)=\gamma_{h}^{-}(t), & \ell_{h}^{-}(t, 1)=\gamma^{-}(t) .
\end{array}
$$

Following the notation of Lemma 2.8 we also write $\Phi_{\ell_{h}^{ \pm}}(t, s):=\left(t, \ell_{h}^{ \pm}(t, s)\right)$. We define the maps $\left(\Psi_{k}^{h}\right) \subset \operatorname{semicart}\left(\mathrm{R} ; \Gamma^{-}, \Gamma^{+}\right)$as

$$
\Psi_{k}^{h}(t, s):= \begin{cases}\Phi_{\ell_{h}^{+}}(t, 4 s-3) & \text { if } t \in(a, b), s \in[1 / 2,1), \\ \Phi_{k}^{h}(t, 2 s) & \text { if } t \in(a, b), s \in(-1 / 2,1 / 2), \\ \Phi_{\ell_{h}^{-}}(t,-4 s-3) & \text { if } t \in(a, b), s \in(-1,-1 / 2] .\end{cases}
$$


We have, using also (4.6),

$$
\begin{aligned}
& \int_{\mathrm{R}}\left|\partial_{t} \Psi_{k}^{h} \wedge \partial_{s} \Psi_{k}^{h}\right| \mathrm{d} t \mathrm{~d} s \\
& =\int_{\mathrm{R}}\left|\partial_{t} \Phi_{k}^{h} \wedge \partial_{s} \Phi_{k}^{h}\right| \mathrm{d} t \mathrm{~d} s+\int_{\mathrm{R}}\left|\partial_{t} \Phi_{\ell_{h}^{+}} \wedge \partial_{s} \Phi_{\ell_{h}^{+}}\right| \mathrm{d} t \mathrm{~d} s+\int_{\mathrm{R}}\left|\partial_{t} \Phi_{\ell_{h}^{-}} \wedge \partial_{s} \Phi_{\ell_{h}^{-}}\right| \mathrm{d} t \mathrm{~d} s \\
& \stackrel{k \rightarrow+\infty}{\longrightarrow} m\left(\mathrm{R} ; \Gamma_{h}^{-}, \Gamma_{h}^{+}\right)+\int_{\mathrm{R}}\left|\partial_{t} \Phi_{\ell_{h}^{+}} \wedge \partial_{s} \Phi_{\ell_{h}^{+}}\right| \mathrm{d} t \mathrm{~d} s+\int_{\mathrm{R}}\left|\partial_{t} \Phi_{\ell_{h}^{-}} \wedge \partial_{s} \Phi_{\ell_{h}^{-}}\right| \mathrm{d} t \mathrm{~d} s .
\end{aligned}
$$

Now, recalling inequality (4.5) and our first assumption, we have

$$
\begin{aligned}
& \int_{\mathrm{R}}\left|\partial_{t} \Phi_{\ell_{h}^{+}} \wedge \partial_{s} \Phi_{\ell_{h}^{+}}\right| \mathrm{d} t \mathrm{~d} s+\int_{\mathrm{R}}\left|\partial_{t} \Phi_{\ell_{h}^{-}} \wedge \partial_{s} \Phi_{\ell_{h}^{-}}\right| \mathrm{d} t \mathrm{~d} s \\
& \quad \leq C\left(\left\|\gamma_{h}^{+}-\gamma^{+}\right\|_{L^{1}\left((a, b) ; \mathbb{R}^{2}\right)}+\left\|\gamma_{h}^{-}-\gamma^{-}\right\|_{L^{1}\left((a, b) ; \mathbb{R}^{2}\right)}\right)\left(1+C_{1}\right),
\end{aligned}
$$

and the right hand side is infinitesimal as $h \rightarrow+\infty$ by our second assumption. Hence, we can select a subsequence $\left(k_{h}\right)$ and obtain a sequence $\left(\Psi_{k_{h}}^{h}\right) \subset \operatorname{semicart}\left(\mathrm{R} ; \Gamma^{-}, \Gamma^{+}\right)$so that

$$
\liminf _{h \rightarrow+\infty} \int_{\mathrm{R}}\left|\partial_{t} \Psi_{k_{h}}^{h} \wedge \partial_{s} \Psi_{k_{h}}^{h}\right| \mathrm{d} t \mathrm{~d} s=\liminf _{h \rightarrow+\infty} m\left(\mathrm{R} ; \Gamma_{h}^{-}, \Gamma_{h}^{+}\right)
$$

The inclusion $\Psi_{k_{h}}^{h} \in \operatorname{semicart}\left(\mathrm{R} ; \Gamma^{-}, \Gamma^{+}\right)$implies that

$$
\int_{\mathrm{R}}\left|\partial_{t} \Psi_{k_{h}}^{h} \wedge \partial_{s} \Psi_{k_{h}}^{h}\right| \mathrm{d} t \mathrm{~d} s \geq m\left(R ; \Gamma^{-}, \Gamma^{+}\right),
$$

and the assertion of the lemma follows.

The last result that we need before proving Theorem 4.2 provides an estimate from below of the area of the graph of a sufficiently smooth map on a strip.

Lemma 4.6 (Lower bound of area on a strip) Let $\varepsilon \in(0,1)$ and $\mathrm{R}_{\varepsilon}:=(a, b) \times(-\varepsilon, \varepsilon)$. Given a map $\mathbf{v} \in \operatorname{Lip}\left(\mathrm{R}_{\varepsilon} ; \mathbb{R}^{2}\right)$, let $\Gamma_{\varepsilon}^{ \pm}$denote the graphs on $[a, b]$ of the sections $\mathbf{v}(\cdot, \pm \varepsilon) \in$ $\operatorname{Lip}\left([a, b] ; \mathbb{R}^{2}\right)$. Then

$$
\mathcal{A}\left(\mathbf{v}, \mathrm{R}_{\varepsilon}\right) \geq m\left(\mathrm{R} ; \Gamma_{\varepsilon}^{-}, \Gamma_{\varepsilon}^{+}\right) .
$$

Proof Set $\mathbf{v}=\left(v_{1}, v_{2}\right)$. Neglecting the constant 1 and the term $\left|\partial_{t} \mathbf{v}\right|^{2}$ in the expression of $|\mathcal{M}(\nabla \mathbf{v})|$, we deduce

$$
\mathcal{A}\left(\mathbf{v}, \mathrm{R}_{\varepsilon}\right) \geq \int_{\mathrm{R}_{\varepsilon}} \sqrt{\left|\partial_{s} \mathbf{v}\right|^{2}+\left(\partial_{t} v_{1} \partial_{s} v_{2}-\partial_{s} v_{1} \partial_{t} v_{2}\right)^{2}} \mathrm{~d} t \mathrm{~d} s .
$$

On the other hand we can define the map $\Phi \in \operatorname{semicart}\left(\mathrm{R} ; \Gamma_{\varepsilon}^{-}, \Gamma_{\varepsilon}^{+}\right)$as

$$
\Phi(t, s):=(t, \mathbf{v}(t, \varepsilon s)), \quad(t, s) \in \mathrm{R},
$$

and (2.2) shows that $\int_{\mathrm{R}}\left|\partial_{t} \Phi \wedge \partial_{S} \Phi\right| \mathrm{d} t \mathrm{~d} s$ equals the right hand side of (4.7). Hence

$$
\mathcal{A}\left(\mathbf{v}, \mathrm{R}_{\varepsilon}\right) \geq \int_{\mathrm{R}}\left|\partial_{t} \Phi \wedge \partial_{s} \Phi\right| \mathrm{d} t \mathrm{~d} s \geq m\left(\mathrm{R} ; \Gamma_{\varepsilon}^{-}, \Gamma_{\varepsilon}^{+}\right) .
$$

Now, we can prove Theorem 4.2. 
Proof Recalling the properties of the sequence $\left(\mathbf{u}_{h}\right)$, we can choose an infinitesimal sequence $\left(\varepsilon_{h}\right)$ of positive numbers such that $\mathrm{R}_{\varepsilon_{h}}:=(a, b) \times\left(-\varepsilon_{h}, \varepsilon_{h}\right) \supseteq N_{h}$. We have

$$
\mathcal{A}\left(\mathbf{u}_{h}, \mathrm{R}\right)=\mathcal{A}\left(\mathbf{u}, \mathrm{R} \backslash \mathrm{R}_{\varepsilon_{h}}\right)+\mathcal{A}\left(\mathbf{u}_{h}, \mathrm{R}_{\varepsilon_{h}}\right) .
$$

Set $\gamma_{h}^{ \pm}(\cdot):=\mathbf{u}_{h}\left(\cdot, \pm \varepsilon_{h}\right)$ and $\gamma^{ \pm}:=\gamma^{ \pm}[\mathbf{u}]$. We observe that, by assumption, $\gamma_{h}^{ \pm}=$ $\mathbf{u}\left(\cdot, \pm \varepsilon_{h}\right)$ and thus $\gamma_{h}^{ \pm}$and $\gamma$ satisfy the hypotheses of Lemma 4.5. Hence, applying also Lemma 4.6, we get

$$
\begin{aligned}
\liminf _{h \rightarrow+\infty} \mathcal{A}\left(\mathbf{u}_{h}, \mathrm{R}\right) & \geq \liminf _{h \rightarrow+\infty}\left[\mathcal{A}\left(\mathbf{u}, \mathrm{R} \backslash \mathrm{R}_{\varepsilon_{h}}\right)+m\left(\mathrm{R} ; \Gamma_{h}^{-}, \Gamma_{h}^{+}\right)\right] \\
& \geq \int_{\mathrm{R}}|\mathcal{M}(\nabla \mathbf{u})| \mathrm{d} t \mathrm{~d} s+m\left(\mathrm{R} ; \Gamma^{-}, \Gamma^{+}\right),
\end{aligned}
$$

that is the thesis.

Remark 4.7 The strategy of the proof of Theorem 4.2 would prove the lower bound (4.1) for any sequence $\left(\mathbf{u}_{h}\right) \subset \mathcal{C}^{1}\left(\mathrm{R} ; \mathbb{R}^{2}\right)$ converging to $\mathbf{u}$ in $L^{1}\left(\mathrm{R} ; \mathbb{R}^{2}\right)$, if we would be able to remove the bound on the $L^{\infty}$-norm of $\dot{\gamma}_{h}^{ \pm}$in the hypotheses of Lemma 4.5. Indeed, as a consequence of Fubini's theorem, the convergence of $\left(\mathbf{u}_{h}\right)$ to $\mathbf{u}$ in $L^{1}\left(\mathrm{R} ; \mathbb{R}^{2}\right)$ implies that $\mathbf{u}_{h}(\cdot, \varepsilon) \rightarrow \mathbf{u}(\cdot, \varepsilon)$ in $L^{1}\left((a, b) ; \mathbb{R}^{2}\right)$ for almost every level $\varepsilon \in(0,1)$.

Lemma 4.5 is in some sense coherent with the lower semicontinuity of the area of solutions of Plateau's problem (when $\Gamma_{h}$ and $\Gamma$ are Jordan curves); indeed lower semicontinuity is usually guaranteed when $\Gamma_{h} \rightarrow \Gamma$ in the sense of Fréchet, $[8,13]$, that would be implied by our hypotheses. On the other hand, in Example 4.8 we exhibit a sequence $\left(\Gamma_{h}\right)$ of curves, union of two Lipschitz graphs, converging in $L^{1}$ to a union $\Gamma$ of two Lipschitz graphs, for which the lower semicontinuity fails. In this context $\Gamma_{h} \rightarrow \Gamma$ in $L^{1}$ means that $\Gamma_{h}:=$ $\operatorname{graph}\left(\gamma_{h}^{-}\right) \cup \operatorname{graph}\left(\gamma_{h}^{+}\right), \Gamma:=\operatorname{graph}\left(\gamma^{-}\right) \cup \operatorname{graph}\left(\gamma^{+}\right), \gamma_{h}^{ \pm}, \gamma^{ \pm} \in \operatorname{Lip}\left([a, b] ; \mathbb{R}^{2}\right)$, and $\gamma_{h}^{ \pm} \rightarrow \gamma^{ \pm}$in $L^{1}\left((a, b) ; \mathbb{R}^{2}\right)$ as $h \rightarrow+\infty$.

Example 4.8 (Lack of $L^{1}$-lower semicontinuity for the Plateau's problem) Let $\Gamma \subset \mathbb{R}^{3}$ be a closed simple rectifiable curve. As already recalled in Remark 4.7, it is known that $a(\cdot)$ is Fréchet lower semicontinuous. We show here that if $\Gamma$ is union of the graphs of $\gamma^{ \pm} \in \operatorname{Lip}\left([a, b] ; \mathbb{R}^{2}\right)$, it may happen that

$$
a(\Gamma)>\liminf _{h \rightarrow+\infty} a\left(\Gamma_{h}\right),
$$

where $\left(\Gamma_{h}\right)$ is a sequence of closed simple space curves, $\Gamma_{h}=\operatorname{graph}\left(\gamma_{h}^{-}\right) \cup \operatorname{graph}\left(\gamma_{h}^{+}\right)$, and $\gamma_{h}^{ \pm} \rightarrow \gamma^{ \pm}$in $L^{1}\left((a, b) ; \mathbb{R}^{2}\right)$ as $h \rightarrow+\infty$.

Indeed, choose the maps $\gamma^{ \pm}$as in (2.10). Let us define the maps $\gamma_{h}^{ \pm} \in \operatorname{Lip}\left([a, b] ; \mathbb{R}^{2}\right)$ converging to $\gamma^{ \pm}$in $L^{1}\left((a, b) ; \mathbb{R}^{2}\right)$ as

$$
\gamma_{h}^{-}(t)=(1,0)=\gamma^{-}(t), \quad \gamma_{h}^{+}(t)=\rho\left(\cos \left(\theta_{h}(t)\right), \sin \left(\theta_{h}(t)\right)\right)+(1-\rho, 0),
$$

where

$$
\theta_{h}(t):= \begin{cases}\theta(t) & \text { if } t \in\left[a, b-h^{-1}\right], \\ -2 \pi \frac{b-a-h^{-1}}{b-a} h(t-b) & \text { if } t \in\left(b-h^{-1}, b\right],\end{cases}
$$

with $\theta$ defined in (2.11). Hence, in the short interval $\left(b-h^{-1}, b\right)$, the path made by $\gamma_{h}^{+}$ is the same as the path it makes in $\left(a, b-h^{-1}\right)$, with reversed orientation. The curve $\Gamma_{h}$ is represented in Fig. 3. For any $h \in \mathbb{N}$ there exists an immersion of the disk, mapping the boundary of the disk onto $\Gamma_{h}$ whose image lies on the lateral boundary of the cylinder 
Fig. 3 One element of the sequence $\left(\Gamma_{h}\right)$ defined in Example 4.8 that approximates in $L^{1}$ sense the curve $\Gamma$ defined in Example 2.13, and a disk-type surface with boundary $\Gamma_{h}$ that lies on the cylinder

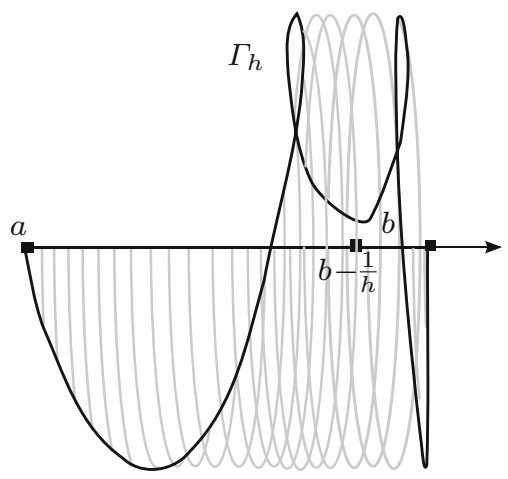

$[a, b] \times B_{\rho}((1-\rho, 0))$; hence for any $h \in \mathbb{N}$ we have $a\left(\Gamma_{h}\right) \leq 2 \pi \rho(b-a)$ that, for $\rho$ large enough, gives (4.8), see Example 2.13.

This example does not exclude the lower semicontinuity of $m\left(\mathrm{R} ; \Gamma^{-}, \Gamma^{+}\right)$with respect to the $L^{1}$-convergence. Indeed the limit of the areas of the surfaces represented in Fig. 3 is the area of the surface represented in the first picture of Fig. 2.

\subsection{Proof of Theorem 4.3}

In order to prove Theorem 4.3 we need the following technical result, inspired by [1, Proposition 7.3], that provides a way to interpolate two maps on a strip, by controlling the amount of area of the interpolating map with the thickness of the strip.

Proposition 4.9 (Interpolation, I) Let $\left(\mathbf{u}_{h}\right) \subset \operatorname{Lip}\left(\mathrm{R}^{+} ; \mathbb{R}^{2}\right)$ be a sequence converging to $\mathbf{u} \in \operatorname{Lip}\left(\mathrm{R}^{+} ; \mathbb{R}^{2}\right)$ in $L^{1}\left(\mathrm{R}^{+} ; \mathbb{R}^{2}\right)$. Let $\varepsilon_{o} \in(0,1)$ be fixed, such that $\partial_{t} \mathbf{u}(\cdot, s)_{\mid s=\varepsilon_{o}}$ exists almost everywhere in $(a, b)$. Let $\varepsilon_{i} \in\left(0, \varepsilon_{o}\right)$ be such that:

(i) $\left\|\mathbf{u}_{h}\left(\cdot, \varepsilon_{i}\right)-\mathbf{u}\left(\cdot, \varepsilon_{i}\right)\right\|_{L^{\infty}\left((a, b) ; \mathbb{R}^{2}\right)} \rightarrow 0$ as $h \rightarrow+\infty$;

(ii) $\partial_{t} \mathbf{u}_{h}(\cdot, s)_{\mid s=\varepsilon_{i}}$ exists almost everywhere in $(a, b)$ for any $h \in \mathbb{N}$;

(iii) $\liminf _{h \rightarrow+\infty}\left\|\partial_{t} \mathbf{u}_{h}\left(\cdot, \varepsilon_{i}\right)\right\|_{L^{1}\left((a, b) ; \mathbb{R}^{2}\right)} \leq M$, where the constant $M$ may depend on $\varepsilon_{i}$.

Then the sequence $\left(\mathbf{v}_{h}\right) \subset \operatorname{Lip}\left(\mathrm{R}^{+} ; \mathbb{R}^{2}\right)$ defined as

$$
\mathbf{v}_{h}(t, s):= \begin{cases}\mathbf{u}(t, s) & \text { if } t \in(a, b), s>\varepsilon_{o}, \\ \frac{\varepsilon_{o}-s}{\varepsilon_{o}-\varepsilon_{i}} \mathbf{u}_{h}\left(t, \varepsilon_{i}\right)+\frac{s-\varepsilon_{i}}{\varepsilon_{o}-\varepsilon_{i}} \mathbf{u}\left(t, \varepsilon_{o}\right) & \text { if } t \in(a, b), s \in\left[\varepsilon_{i}, \varepsilon_{o}\right], \\ \mathbf{u}_{h}(t, s) & \text { if } t \in(a, b), s<\varepsilon_{i}\end{cases}
$$

satisfies

$$
\liminf _{h \rightarrow+\infty} \mathcal{A}\left(\mathbf{v}_{h},(a, b) \times\left(\varepsilon_{i}, \varepsilon_{o}\right)\right) \leq C[1+M]\left|\varepsilon_{o}-\varepsilon_{i}\right|,
$$

where $C>0$ depends on $\operatorname{lip}(\mathbf{u})$ and $b-a$, and is independent of $\varepsilon_{o}$ and $\varepsilon_{i}$.

Proof Let $S_{\varepsilon_{i}}^{\varepsilon_{o}}:=(a, b) \times\left(\varepsilon_{i}, \varepsilon_{o}\right)$. The Jacobian matrix of $\mathbf{v}_{h}$ at almost every $(t, s) \in S_{\varepsilon_{i}}^{\varepsilon_{o}}$ is:

$$
\frac{1}{\varepsilon_{o}-\varepsilon_{i}}\left(\left(\varepsilon_{o}-s\right) \partial_{t} \mathbf{u}_{h}\left(t, \varepsilon_{i}\right)+\left(s-\varepsilon_{i}\right) \partial_{t} \mathbf{u}\left(t, \varepsilon_{o}\right) \mid \mathbf{u}\left(t, \varepsilon_{o}\right)-\mathbf{u}_{h}\left(t, \varepsilon_{i}\right)\right) \text {. }
$$


We control the area of the graph of $\mathbf{v}_{h}$ in $S_{\varepsilon_{i}}^{\varepsilon_{o}}$ as

$$
\mathcal{A}\left(\mathbf{v}_{h}, S_{\varepsilon_{i}}^{\varepsilon_{o}}\right) \leq C \int_{S_{\varepsilon_{i}}^{\varepsilon_{o}}}\left[\left[1+\left|\partial_{t} \mathbf{v}_{h}\right|+\left|\partial_{s} \mathbf{v}_{h}\right|+\left|\operatorname{det} \nabla \mathbf{v}_{h}\right|\right] \mathrm{d} t \mathrm{~d} s,\right.
$$

where $C>0$ is an absolute constant. We estimate each of the four integrals on the right hand side of (4.11) as follows.

- The first term is obviously bounded by $\left(\varepsilon_{o}-\varepsilon_{i}\right)(b-a)$.

- Concerning the second term, we have

$$
\begin{aligned}
\int_{\varepsilon_{i}}^{\varepsilon_{o}} \int_{a}^{b}\left|\partial_{t} \mathbf{v}_{h}\right| \mathrm{d} t \mathrm{~d} s & \leq\left(\varepsilon_{o}-\varepsilon_{i}\right) \int_{a}^{b}\left(\left|\partial_{t} \mathbf{u}_{h}\left(t, \varepsilon_{i}\right)\right|+\left|\partial_{t} \mathbf{u}\left(t, \varepsilon_{o}\right)\right|\right) \mathrm{d} t \\
& \leq\left(\varepsilon_{o}-\varepsilon_{i}\right)\left[\int_{a}^{b}\left|\partial_{t} \mathbf{u}_{h}\left(t, \varepsilon_{i}\right)\right| \mathrm{d} t+\operatorname{lip}(\mathbf{u})(b-a)\right]
\end{aligned}
$$

- Similarly, for the third term we have

$$
\begin{aligned}
\int_{\varepsilon_{i}}^{\varepsilon_{o}} \int_{a}^{b}\left|\partial_{s} \mathbf{v}_{h}(t, s)\right| \mathrm{d} t \mathrm{~d} s & \leq \int_{a}^{b}\left[\left|\mathbf{u}\left(t, \varepsilon_{o}\right)-\mathbf{u}\left(t, \varepsilon_{i}\right)\right|+\left|\mathbf{u}\left(t, \varepsilon_{i}\right)-\mathbf{u}_{h}\left(t, \varepsilon_{i}\right)\right|\right] \mathrm{d} t \\
& \leq \operatorname{lip}(\mathbf{u})(b-a)\left(\varepsilon_{o}-\varepsilon_{i}\right)+|| \mathbf{u}\left(\cdot, \varepsilon_{i}\right)-\mathbf{u}_{h}\left(\cdot, \varepsilon_{i}\right) \|_{L^{1}\left((a, b) ; \mathbb{R}^{2}\right)} .
\end{aligned}
$$

- Concerning the term with the determinant:

$$
\begin{aligned}
& \int_{\varepsilon_{i}}^{\varepsilon_{o}} \int_{a}^{b}\left|\operatorname{det} \nabla \mathbf{v}_{h}(t, s)\right| \mathrm{d} t \mathrm{~d} s \\
& \quad \leq 2 \int_{a}^{b}\left|\mathbf{u}\left(t, \varepsilon_{o}\right)-\mathbf{u}_{h}\left(t, \varepsilon_{i}\right)\right|\left(\left|\partial_{t} \mathbf{u}_{h}\left(t, \varepsilon_{i}\right)\right|+\left|\partial_{t} \mathbf{u}\left(t, \varepsilon_{o}\right)\right|\right) \mathrm{d} t \\
& \quad=2 \int_{a}^{b}\left|\mathbf{u}\left(t, \varepsilon_{o}\right)-\mathbf{u}_{h}\left(t, \varepsilon_{i}\right)\right|\left|\partial_{t} \mathbf{u}\left(t, \varepsilon_{o}\right)\right| \mathrm{d} t+2 \int_{a}^{b}\left|\mathbf{u}\left(t, \varepsilon_{o}\right)-\mathbf{u}_{h}\left(t, \varepsilon_{i}\right)\right|\left|\partial_{t} \mathbf{u}_{h}\left(t, \varepsilon_{i}\right)\right| \mathrm{d} t \\
& =: \mathrm{I}_{h}+\mathrm{II}_{h} .
\end{aligned}
$$

We have

$$
\begin{aligned}
\mathrm{I}_{h} & \leq 2 \operatorname{lip}(\mathbf{u}) \int_{a}^{b}\left(\left|\mathbf{u}\left(t, \varepsilon_{o}\right)-\mathbf{u}\left(t, \varepsilon_{i}\right)\right|+\left|\mathbf{u}\left(t, \varepsilon_{i}\right)-\mathbf{u}_{h}\left(t, \varepsilon_{i}\right)\right|\right) \mathrm{d} t \\
& \leq 2(\operatorname{lip}(\mathbf{u}))^{2}(b-a)\left(\varepsilon_{o}-\varepsilon_{i}\right)+2 \operatorname{lip}(\mathbf{u})\left\|\mathbf{u}\left(\cdot, \varepsilon_{i}\right)-\mathbf{u}_{h}\left(\cdot, \varepsilon_{i}\right)\right\|_{L^{1}\left((a, b) ; \mathbb{R}^{2}\right)} .
\end{aligned}
$$

Next

$$
\begin{aligned}
\mathrm{II}_{h} & \leq 2 \int_{a}^{b}\left|\mathbf{u}\left(t, \varepsilon_{o}\right)-\mathbf{u}\left(t, \varepsilon_{i}\right)\right|\left|\partial_{t} \mathbf{u}_{h}\left(t, \varepsilon_{i}\right)\right| \mathrm{d} t+2 \int_{a}^{b}\left|\mathbf{u}\left(t, \varepsilon_{i}\right)-\mathbf{u}_{h}\left(t, \varepsilon_{i}\right)\right|\left|\partial_{t} \mathbf{u}_{h}\left(t, \varepsilon_{i}\right)\right| \mathrm{d} t \\
& \leq 2\left(\operatorname{lip}(\mathbf{u})\left(\varepsilon_{o}-\varepsilon_{i}\right)+\left\|\mathbf{u}\left(\cdot, \varepsilon_{i}\right)-\mathbf{u}_{h}\left(\cdot, \varepsilon_{i}\right)\right\|_{L^{\infty}\left((a, b) ; \mathbb{R}^{2}\right)}\right) \int_{a}^{b}\left|\partial_{t} \mathbf{u}_{h}\left(t, \varepsilon_{i}\right)\right| \mathrm{d} t .
\end{aligned}
$$

Finally, using (4.12), (4.13), (4.14) and (4.15) we get:

$$
\begin{aligned}
\mathcal{A}\left(\mathbf{v}_{h}, S_{\varepsilon_{i}}^{\varepsilon_{o}}\right) \leq & C\left(\varepsilon_{o}-\varepsilon_{i}\right)\left[1+\left\|\mathbf{u}\left(\cdot, \varepsilon_{i}\right)-\mathbf{u}_{h}\left(\cdot, \varepsilon_{i}\right)\right\|_{L^{1}\left((a, b) ; \mathbb{R}^{2}\right)}\right. \\
& \left.+\left(1+\left\|\mathbf{u}\left(\cdot, \varepsilon_{i}\right)-\mathbf{u}_{h}\left(\cdot, \varepsilon_{i}\right)\right\|_{L^{\infty}\left((a, b) ; \mathbb{R}^{2}\right)}\right) \int_{a}^{b}\left|\partial_{t} \mathbf{u}_{h}\left(t, \varepsilon_{i}\right)\right| \mathrm{d} t\right] .
\end{aligned}
$$


Using hypotheses (i)-(iii), and passing to the limit, we get

$$
\liminf _{h \rightarrow+\infty} \mathcal{A}\left(\mathbf{v}_{h}, S_{\varepsilon_{i}}^{\varepsilon_{o}}\right) \leq C[1+M]\left(\varepsilon_{o}-\varepsilon_{i}\right),
$$

where $C$ depends just on $b-a$ and $\operatorname{lip}(\mathbf{u})$.

We are now in the position to prove Theorem 4.3.

Proof We can suppose that $\mathcal{A}\left(\mathbf{u}_{h}, \mathrm{R}\right)$ is uniformly bounded with respect to $h \in \mathbb{N}$, otherwise the result is trivial. Moreover, passing to a not relabeled subsequence, we can suppose also that there exist

$$
\lim _{h \rightarrow+\infty} \mathcal{A}\left(\mathbf{u}_{h}, \mathrm{R}\right)<+\infty, \quad \lim _{h \rightarrow+\infty} \mathcal{A}\left(\mathbf{u}_{h}, \mathrm{R}^{+}\right)<+\infty, \quad \lim _{h \rightarrow+\infty} \mathcal{A}\left(\mathbf{u}_{h}, \mathrm{R}^{-}\right)<+\infty .
$$

Since $\mathbf{u}_{h} \rightarrow \mathbf{u}$ uniformly on every compact set of $\mathrm{R}^{+}$as $h \rightarrow+\infty$, hypothesis $(i)$ of Proposition 4.9 is verified for any choice of the level $\varepsilon_{1} \in(0,1)$. Using Fatou's lemma we get

$$
\int_{0}^{1} \liminf _{h \rightarrow+\infty}\left(\int_{a}^{b}\left|\partial_{t} \mathbf{u}_{h}(t, s)\right| \mathrm{d} t\right) \mathrm{d} s \leq \liminf _{h \rightarrow+\infty} \mathcal{A}\left(\mathbf{u}_{h}, \mathrm{R}^{+}\right)<+\infty .
$$

Thus we can select a level $\varepsilon_{1} \in(0,1)$, a subsequence $\left(\mathbf{u}_{h_{j}}\right)$ and a constant $M\left(\varepsilon_{1}\right)$ both depending on $\varepsilon_{1}$, such that

$$
\lim _{j \rightarrow+\infty} \int_{a}^{b}\left|\partial_{t} \mathbf{u}_{h_{j}}\left(t, \varepsilon_{1}\right)\right| \mathrm{d} t \leq M\left(\varepsilon_{1}\right) .
$$

Repeating the argument a countably number of times and using the same procedure on $\mathrm{R}^{-}$, we can select a subsequence $\left(\mathbf{u}_{h_{j}}\right)$ of $\left(\mathbf{u}_{h}\right)$, and an infinitesimal sequence $\left(\varepsilon_{k}\right)$ of positive levels such that $\mathbf{u}_{h_{j}}\left(\cdot, \pm \varepsilon_{k}\right)$ satisfies the hypotheses (i)-(iii) of Proposition 4.9.

Let us choose also an infinitesimal sequence $\left(\delta_{k}\right)$ of positive numbers such that

$$
\delta_{k} M\left(\varepsilon_{k}\right) \stackrel{k \rightarrow+\infty}{\longrightarrow} 0
$$

and such that $\partial_{t} \mathbf{u}(t, s)_{\mid s= \pm\left(\varepsilon_{k}+\delta_{k}\right)}$ exists for almost every $t \in(a, b)$ for any $k \in \mathbb{N}$.

Now, we define the maps $\mathbf{v}_{h_{j}}^{k}$ similarly to (4.9):

$\mathbf{v}_{h_{j}}^{k}(t, s):= \begin{cases}\mathbf{u}(t, s) & \text { if } t \in(a, b), \varepsilon_{k}+\delta_{k}<|s|<1, \\ \frac{\varepsilon_{k}+\delta_{k}-s}{\delta_{k}} \mathbf{u}_{h_{j}}\left(t, \varepsilon_{k}\right)+\frac{s-\varepsilon_{k}}{\delta_{k}} \mathbf{u}\left(t, \varepsilon_{k}+\delta_{k}\right) & \text { if } t \in(a, b), \varepsilon_{k} \leq s \leq \varepsilon_{k}+\delta_{k}, \\ \frac{\varepsilon_{k}+\delta_{k}+s}{\delta_{k}} \mathbf{u}_{h_{j}}\left(t,-\varepsilon_{k}\right)+\frac{-s-\varepsilon_{k}}{\delta_{k}} \mathbf{u}\left(t,-\left(\varepsilon_{k}+\delta_{k}\right)\right) & \text { if } t \in(a, b),-\left(\varepsilon_{k}+\delta_{k}\right) \leq s \leq-\varepsilon_{k}, \\ \mathbf{u}_{h_{j}}(t, s) & \text { if } t \in(a, b),|s|<\varepsilon_{k} .\end{cases}$

We claim that for any $k \in \mathbb{N}$ we have

$$
\liminf _{j \rightarrow+\infty} \mathcal{A}\left(\mathbf{v}_{h_{j}}^{k}, \mathrm{R}\right) \leq \lim _{h \rightarrow+\infty} \mathcal{A}\left(\mathbf{u}_{h}, \mathrm{R}\right)+C\left[1+M\left(\varepsilon_{k}\right)\right] \delta_{k},
$$

where $C$ is the constant given in (4.10).

For any $\lambda \in(0,1)$ set $\mathrm{R}_{\lambda}^{+}:=(a, b) \times(0, \lambda)$. Without loss of generality we can suppose that there exists $\lim _{h \rightarrow+\infty} \mathcal{A}\left(\mathbf{u}_{h}, \mathrm{R}^{+} \backslash \mathrm{R}_{\varepsilon_{k}+\delta_{k}}^{+}\right)$for any $k \in \mathbb{N}$. Using the same notation as in the proof of Proposition 4.9, and since $\mathcal{A}\left(\mathbf{u}_{h_{j}}, \mathrm{R}_{\varepsilon_{k}}^{+}\right) \leq \mathcal{A}\left(\mathbf{u}_{h_{j}}, \mathrm{R}^{+}\right)-\mathcal{A}\left(\mathbf{u}_{h_{j}}, \mathrm{R}^{+} \backslash \mathrm{R}_{\varepsilon_{k}+\delta_{k}}^{+}\right)$, we get: 


$$
\begin{aligned}
\mathcal{A}\left(\mathbf{v}_{h_{j}}^{k}, \mathrm{R}^{+}\right)= & \mathcal{A}\left(\mathbf{u}, \mathrm{R}^{+} \backslash \mathrm{R}_{\varepsilon_{k}+\delta_{k}}^{+}\right)+\mathcal{A}\left(\mathbf{v}_{h_{j}}^{k}, S_{\varepsilon_{k}}^{\varepsilon_{k}+\delta_{k}}\right)+\mathcal{A}\left(\mathbf{u}_{h_{j}}, \mathrm{R}_{\varepsilon_{k}}^{+}\right) \\
\leq & \mathcal{A}\left(\mathbf{u}, \mathrm{R}^{+} \backslash \mathrm{R}_{\varepsilon_{k}+\delta_{k}}^{+}\right)-\mathcal{A}\left(\mathbf{u}_{h_{j}}, \mathrm{R}^{+} \backslash \mathrm{R}_{\varepsilon_{k}+\delta_{k}}^{+}\right)+\mathcal{A}\left(\mathbf{v}_{h_{j}}^{k}, S_{\varepsilon_{k}}^{\varepsilon_{k}+\delta_{k}}\right) \\
& +\mathcal{A}\left(\mathbf{u}_{h_{j}}, \mathrm{R}^{+}\right) .
\end{aligned}
$$

Passing to the limit as $j \rightarrow+\infty$, recalling that $\mathcal{A}\left(\cdot, \mathrm{R}^{+} \backslash \mathrm{R}_{\varepsilon_{k}+\delta_{k}}^{+}\right)$is lower semicontinuous, using (4.10), and making similar computations also in $\mathrm{R}^{-}$, we get claim (4.18).

Finally, the proof of (4.2) is concluded by remembering (4.17), choosing a suitable subsequence $\left(k_{h_{j}}\right)$ and defining $\mathbf{v}_{h_{j}}:=\mathbf{v}_{h_{j}}$.

\section{Condition II: upper bound}

In this short section and in Sect. 6 we discuss the case where $\Omega$ and $\mathbf{u}$ satisfy condition II. In Proposition 5.1, following the strategy of [4], we prove the upper bound in Theorem 1.4 (see inequality (5.2)). We recall that $D=\left[\left[\sigma^{-}, \sigma^{+}\right]\right]$is the (fixed) domain defined in Definition 2.5. Similarly to the case when $\Omega$ and $\mathbf{u}$ satisfy condition I, this upper bound implies also that $\overline{\mathcal{A}}_{S}(\mathbf{u}, \Omega) \leq m\left(D ; \Gamma^{-}, \Gamma^{+}\right)$, since in general $\overline{\mathcal{A}}(\mathbf{u}, \Omega) \leq \overline{\mathcal{A}}^{\infty}(\mathbf{u}, \Omega)$. In Sect. 7 we describe some examples where the latter inequality is strict.

The proof of the next proposition is similar to the one in [4, Theorem 4.1]. We briefly report it for the sake of completeness.

Proposition 5.1 (Upper bound, II) Let $\Omega$ and $\mathbf{u}$ satisfy condition II. Then there exists a sequence $\left(\mathbf{u}_{h}\right) \subset H^{1}\left(\Omega ; \mathbb{R}^{2}\right)$ converging to $\mathbf{u}$ in $L^{1}\left(\Omega ; \mathbb{R}^{2}\right)$ and uniformly out of $J_{\mathbf{u}}$, such that

$$
\liminf _{h \rightarrow+\infty} \mathcal{A}\left(\mathbf{u}_{h}, \Omega\right)=\int_{\Omega}|\mathcal{M}(\nabla \mathbf{u})| d t d s+m\left(D ; \Gamma^{-}[\mathbf{u}], \Gamma^{+}[\mathbf{u}]\right) .
$$

Hence

$$
\overline{\mathcal{A}}_{s}^{\infty}(\mathbf{u}, \Omega) \leq m\left(D ; \Gamma^{-}[\mathbf{u}], \Gamma^{+}[\mathbf{u}]\right) .
$$

Remark 5.2 We notice that $m\left(D ; \Gamma^{-}[\mathbf{u}], \Gamma^{+}[\mathbf{u}]\right)$ is well defined; indeed the two traces of $\mathbf{u}$ on the sides of $J_{\mathbf{u}}$ are defined and coincide at the endpoints of the jump, since $J_{\mathbf{u}} \subset \subset \Omega$, and $\mathbf{u} \in W^{1, \infty}\left(\Omega \backslash J_{\mathbf{u}} ; \mathbb{R}^{2}\right)$.

Proof We can suppose without loss of generality that $D=\left[\left[\sigma^{-}, \sigma^{+}\right]\right]$with $\left|\sigma^{ \pm}\right|<1$, compare Definition 2.3, and hence $D \subset \mathrm{R}$. Let $\Phi_{h} \in \operatorname{semicart}\left(D ; \Gamma^{-}, \Gamma^{+}\right)$be such that $\lim _{h \rightarrow+\infty} \int_{D}\left|\partial_{t} \Phi_{h} \wedge \partial_{s} \Phi_{h}\right| d t d s=m\left(D ; \Gamma^{-}, \Gamma^{+}\right)$. For any $\varepsilon \in(0,1)$ we set $\mathrm{R}_{\varepsilon}:=$ $(a, b) \times(-\varepsilon, \varepsilon)$ and $D_{\varepsilon}:=\left[\left[\sigma_{\varepsilon}^{-}, \sigma_{\varepsilon}^{+}\right]\right]$with $\sigma_{\varepsilon}^{ \pm}:=\varepsilon \sigma^{ \pm}$; we define also the map $T_{\varepsilon}$ : $\mathrm{R}_{\varepsilon} \backslash D_{\varepsilon} \rightarrow \mathrm{R}_{\varepsilon} \backslash(a, b) \times\{0\}$ as follows:

$$
T_{\varepsilon}(t, s):= \begin{cases}\left(t, \frac{s-\varepsilon \sigma^{+}(t)}{1-\sigma^{+}(t)}\right) & \text { if }(t, s) \in\left(R_{\varepsilon} \backslash D_{\varepsilon}\right) \cap\{s>0\}, \\ \left.t, \frac{s-\varepsilon \sigma^{-}(t)}{1+\sigma^{-}(t)}\right) & \text { if }(t, s) \in\left(R_{\varepsilon} \backslash D_{\varepsilon}\right) \cap\{s<0\} .\end{cases}
$$

Next, let us consider the sequence $\left(\mathbf{u}_{\varepsilon}^{h}\right) \subset H^{1}\left(\Omega ; \mathbb{R}^{2}\right)$ given by

$$
\mathbf{u}_{\varepsilon}^{h}(t, s):= \begin{cases}\mathbf{u}(t, s) & \text { if }(t, s) \in \Omega \backslash \mathrm{R}_{\varepsilon} \\ \mathbf{u}\left(T_{\varepsilon}(t, s)\right) & \text { if }(t, s) \in \mathrm{R}_{\varepsilon} \backslash \overline{D_{\varepsilon}} \\ \phi_{h}(t, s / \varepsilon) & \text { if }(t, s) \in \overline{D_{\varepsilon}},\end{cases}
$$


where $\Phi_{h}(t, s)=\left(t, \phi_{h}(t, s)\right)$. Since $T_{\varepsilon}$ and its derivatives are bounded by a constant depending only on $\operatorname{lip}\left(\sigma^{ \pm}\right)$, with computations similar to the ones in Proposition 3.1 we get

$$
\lim _{\varepsilon \rightarrow 0^{+}} \mathcal{A}\left(\mathbf{u}_{\varepsilon}^{h}, \Omega\right)=\int_{\Omega}|\mathcal{M}(\nabla \mathbf{u})| \mathrm{d} t \mathrm{~d} s+\int_{D}\left|\partial_{t} \Phi_{h} \wedge \partial_{s} \Phi_{h}\right| \mathrm{d} t \mathrm{~d} s .
$$

Hence the required sequence is obtained as $\left(\mathbf{u}_{h}\right):=\left(\mathbf{u}_{\varepsilon_{h}}^{h}\right)$, for a suitable infinitesimal sequence $\left(\varepsilon_{h}\right)$ of positive numbers such that $\mathrm{R}_{\varepsilon_{h}} \subset \Omega$ for every $h \in \mathbb{N}$.

\section{Condition II: lower bound}

In this section we want to prove inequality (6.1) which, coupled with Proposition 5.1, concludes the proof of Theorem 1.4.

Theorem 6.1 (Lower bound, II) Let $\Omega$ and $\mathbf{u}$ satisfy condition II. Let $\left(\mathbf{u}_{h}\right) \subset \operatorname{Lip}\left(\Omega ; \mathbb{R}^{2}\right)$ be a sequence converging to $\mathbf{u}$ in $L^{1}\left(\Omega ; \mathbb{R}^{2}\right)$ and uniformly out of $J_{\mathbf{u}}$. Then

$$
\liminf _{h \rightarrow+\infty} \mathcal{A}\left(\mathbf{u}_{h}, \Omega\right) \geq \int_{\Omega}|\mathcal{M}(\nabla \mathbf{u})| d t d s+m\left(D ; \Gamma^{-}[\mathbf{u}], \Gamma^{+}[\mathbf{u}]\right) .
$$

Hence

$$
\overline{\mathcal{A}}_{s}^{\infty}(\mathbf{u}, \Omega) \geq m\left(D ; \Gamma^{-}[\mathbf{u}], \Gamma^{+}[\mathbf{u}]\right) .
$$

As in Sect. 4, we shall divide the proof in two steps: in the first step we prove the theorem under the further hypothesis that

$$
\mathbf{u}_{h}=\mathbf{u} \quad \text { in } \Omega \backslash N_{h},
$$

where $\left(N_{h}\right)$ is a decreasing sequence of neighborhoods of $J_{\mathbf{u}}$ such that $\bigcap_{h \in \mathbb{N}} N_{h}=J_{\mathbf{u}}$. In order to prove this step, we shall need the analogous of Lemma 4.5. In the second step we prove that for any sequence $\left(\mathbf{u}_{h}\right)$ converging to $\mathbf{u}$ in $L^{1}\left(\Omega, \mathbb{R}^{2}\right)$ and uniformly out of $J_{\mathbf{u}}$, there exists a sequence $\left(\mathbf{v}_{h}\right) \subset \operatorname{Lip}\left(\Omega ; \mathbb{R}^{2}\right)$ satisfying (6.2) and such that

$$
\liminf _{h \rightarrow+\infty} \mathcal{A}\left(\mathbf{v}_{h}, \Omega\right) \leq \liminf _{h \rightarrow+\infty} \mathcal{A}\left(\mathbf{u}_{h}, \Omega\right) .
$$

In order to prove this step we shall need the analogous of Proposition 4.9.

Let us fix some notation. For any $\varepsilon \in\left(0, \frac{b-a}{2}\right)$, let $\lambda_{\varepsilon}: \mathbb{R}_{t} \rightarrow \mathbb{R}_{t}$ be defined as

$$
\lambda_{\varepsilon}(t):=\frac{b-a-2 \varepsilon}{b-a+2 \varepsilon}(t-(a-\varepsilon))+a+\varepsilon,
$$

so that $\lambda_{\varepsilon}((a-\varepsilon, b+\varepsilon))=(a+\varepsilon, b-\varepsilon)$. The map $\Lambda_{\varepsilon}: \mathbb{R}_{(t, s)}^{2} \rightarrow \mathbb{R}_{(t, s)}^{2}$ is, instead, defined as

$$
\Lambda_{\varepsilon}(t, s)=\left(\lambda_{\varepsilon}(t), s\right) .
$$

We set $O_{\varepsilon}:=\left[\left[\sigma_{\varepsilon}^{-}, \sigma_{\varepsilon}^{+}\right]\right]$, where $\sigma_{\varepsilon}^{ \pm} \in \operatorname{Lip}([a-\varepsilon, b+\varepsilon])$ are such that $\sigma_{\varepsilon}^{-}(a-\varepsilon)=\sigma_{\varepsilon}^{+}(a-\varepsilon)$ and $\sigma_{\varepsilon}^{-}(b+\varepsilon)=\sigma_{\varepsilon}^{+}(b+\varepsilon)$, and such that $\Lambda_{\varepsilon}\left(O_{\varepsilon}\right) \subset \subset D$, see Fig. 4. If necessary, we can require $\partial O_{\varepsilon}$ without horizontal cusps, in the sense that we can suppose that there is a decreasing sequence $\left(t_{i}\right) \subset(a, b)$ converging to $a$, along which $\sigma_{\varepsilon}^{ \pm}$are differentiable, and $\liminf _{i \rightarrow+\infty}\left(\sigma_{\varepsilon}^{+}\right)^{\prime}\left(t_{i}\right)>0, \lim \sup _{i \rightarrow+\infty}\left(\sigma_{\varepsilon}^{-}\right)^{\prime}\left(t_{i}\right)<0$ (and similarly near $t=b$ ).

Lemma 6.2 Let $\gamma^{ \pm} \in \operatorname{Lip}\left([a, b] ; \mathbb{R}^{2}\right)$ be such that $\gamma^{-}(a)=\gamma^{+}(a)$ and $\gamma^{-}(b)=\gamma^{+}(b)$. Let $\left(\varepsilon_{h}\right)$ be an infinitesimal sequence of positive numbers and let $\gamma_{h}^{ \pm} \in \operatorname{Lip}\left(\left[a-\varepsilon_{h}, b+\right.\right.$ $\left.\varepsilon_{h}\right] ; \mathbb{R}^{2}$ ) be maps with the following properties: 


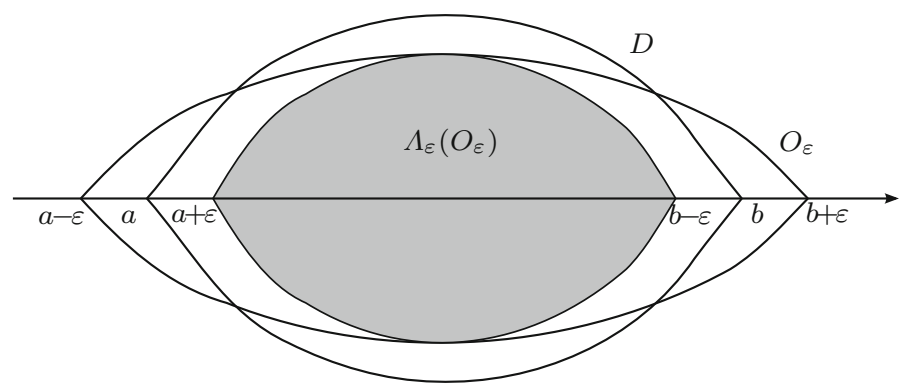

Fig. 4 For any $\varepsilon>0$ small enough, $O_{\varepsilon}=\left[\left[\sigma_{\varepsilon}^{-}, \sigma_{\varepsilon}^{+}\right]\right]$is such that its image through the map $\Lambda_{\varepsilon}$ is compactly contained in the fixed domain $D$

(i) $\gamma_{h}^{-}\left(a-\varepsilon_{h}\right)=\gamma_{h}^{+}\left(a-\varepsilon_{h}\right)$ and $\gamma_{h}^{-}\left(b+\varepsilon_{h}\right)=\gamma_{h}^{+}\left(b+\varepsilon_{h}\right)$ for any $h \in \mathbb{N}$;

(ii) $\lim _{h \rightarrow+\infty} \gamma_{h}^{-}\left(a-\varepsilon_{h}\right)=\gamma^{-}(a)$ and $\lim _{h \rightarrow+\infty} \gamma_{h}^{-}\left(b+\varepsilon_{h}\right)=\gamma^{-}(b)$;

(iii) $\lim _{h \rightarrow+\infty}\left\|\gamma_{h}^{ \pm} \circ \lambda_{\varepsilon_{h}}^{-1}-\gamma^{ \pm}\right\|_{L^{1}\left(\left(a+\varepsilon_{h}, b-\varepsilon_{h}\right) ; \mathbb{R}^{2}\right)}=0$.

Moreover, we also suppose:

(iv) there exists a constant $C_{1}>0$ such that $\left\|\dot{\gamma}_{h}^{ \pm}\right\|_{L^{\infty}\left(\left(a-\varepsilon_{h}, b+\varepsilon_{h}\right) ; \mathbb{R}^{2}\right)} \leq C_{1}$ for any $h \in \mathbb{N}$. Then

$$
m\left(D ; \Gamma^{-}, \Gamma^{+}\right) \leq \liminf _{h \rightarrow+\infty} m\left(O_{\varepsilon_{h}} ; \Gamma_{h}^{-}, \Gamma_{h}^{+}\right),
$$

where $\Gamma^{ \pm}:=\operatorname{graph}\left(\gamma^{ \pm}\right), \Gamma_{h}^{ \pm}:=\operatorname{graph}\left(\gamma_{h}^{ \pm}\right) .^{8}$

Proof Let $\Psi_{h}$ be a semicartesian map in $H^{1}\left(O_{\varepsilon_{h}} ; \mathbb{R}^{3}\right)$ spanning $\Gamma_{h}$ such that

$$
\int_{O_{\varepsilon_{h}}}\left|\partial_{t} \Psi_{h} \wedge \partial_{s} \Psi_{h}\right| \mathrm{d} t \mathrm{~d} s \leq m\left(O_{\varepsilon_{h}} ; \Gamma_{h}^{-}, \Gamma_{h}^{+}\right)+\varepsilon_{h},
$$

with $\Psi_{h}(t, s)=\left(t, \psi_{h}(t, s)\right)$. Let us define $\Phi_{h} \in H^{1}\left(\Lambda_{\varepsilon_{h}}\left(O_{\varepsilon_{h}}\right) ; \mathbb{R}^{3}\right)$ as

$$
\Phi_{h}(t, s):=\left(t, \psi_{h}\left(\lambda_{\varepsilon_{h}}^{-1}(t), s\right)\right)=:\left(t, \phi_{h}(t, s)\right), \quad(t, s) \in \Lambda_{\varepsilon_{h}}\left(O_{\varepsilon_{h}}\right) .
$$

In words, we start from a point in $\Lambda_{\varepsilon_{h}}\left(O_{\varepsilon_{h}}\right)$, we take its image in $O_{\varepsilon_{h}}$ through the dilation $\Lambda_{\varepsilon_{h}}^{-1}$, we pass to its image through the semicartesian map $\Psi_{h}$, and we contract in the $t$-direction through the map $(t, \xi, \eta) \rightarrow\left(\lambda_{\varepsilon_{h}}(t), \xi, \eta\right)$. Recalling (6.5) and since the determinant of the Jacobian of $\Lambda_{\varepsilon_{h}}$ tends to 1 as $h \rightarrow+\infty$, we get

$$
\int_{\Lambda_{\varepsilon_{h}}\left(O_{\varepsilon_{h}}\right)}\left|\partial_{t} \Phi_{h} \wedge \partial_{s} \Phi_{h}\right| \mathrm{d} t \mathrm{~d} s=m\left(O_{\varepsilon_{h}} ; \Gamma_{h}^{-}, \Gamma_{h}^{+}\right)+\mathcal{O}\left(\varepsilon_{h}\right) .
$$

Recalling that $\Lambda_{\varepsilon_{h}}\left(O_{\varepsilon_{h}}\right) \subset \subset D$, we can extend $\Phi_{h}$ to a semicartesian map in $\operatorname{semicart}\left(D ; \Gamma^{-}, \Gamma^{+}\right)$: if $\Lambda\left(O_{\varepsilon_{h}}\right):=\left[\left[\sigma_{\varepsilon_{h}}^{-}, \sigma_{\varepsilon_{h}}^{+}\right]\right]$, we define $\Phi_{h}$ in $S_{\varepsilon_{h}}^{+}:=\{(t, s) \in D$ : $\left.t \in\left(a+\varepsilon_{h}, b-\varepsilon_{h}\right), s \in\left(\sigma_{\varepsilon_{h}}^{+}(t), \sigma^{+}(t)\right)\right\}$ as

$$
\Phi_{h}(t, s):=\left(t, \frac{s-\sigma_{\varepsilon_{h}}^{+}(t)}{\sigma^{+}(t)-\sigma_{\varepsilon_{h}}^{+}(t)} \gamma^{+}(t)+\frac{\sigma^{+}(t)-s}{\sigma^{+}(t)-\sigma_{\varepsilon_{h}}^{+}(t)} \phi_{h}\left(t, \sigma_{\varepsilon_{h}}^{+}(t)\right)\right) .
$$

8 We denote by $m\left(O_{\varepsilon_{h}} ; \Gamma_{h}^{-}, \Gamma_{h}^{+}\right)$the infimum of $\int_{O_{\varepsilon_{h}}}\left|\partial_{t} \Psi \wedge \partial_{S} \Psi\right| \mathrm{d} t \mathrm{~d} s$ among all semicartesian parametrizations $\left(O_{\varepsilon_{h}}, \Psi\right)$ spanning $\Gamma_{h}:=\Gamma_{h}^{-} \cup \Gamma_{h}^{+}$such that $\Psi \in H^{1}\left(O_{\varepsilon_{h}} ; \mathbb{R}^{3}\right)$. 
Similarly, we define $\Phi_{h}$ on $S_{\varepsilon_{h}}^{-}:=\left\{(t, s) \in D: t \in\left(a+\varepsilon_{h}, b-\varepsilon_{h}\right), s \in\left(\sigma^{-}(t), \sigma_{\varepsilon_{h}}^{-}(t)\right)\right\}$. Thanks to hypotheses and recalling Lemma 4.4 (see inequality (4.4)), we deduce

$$
\int_{S_{\varepsilon_{h}}^{-} \cup S_{\varepsilon_{h}}^{+}}\left|\partial_{t} \Phi_{h} \wedge \partial_{s} \Phi_{h}\right| \mathrm{d} t \mathrm{~d} s \stackrel{h \rightarrow+\infty}{\longrightarrow} 0 .
$$

Now, we define $\Phi_{h}$ on the curved triangles $T_{h}^{a}:=\left\{(t, s) \in D: t \in\left(a, a+\varepsilon_{h}\right]\right\}$ and $T_{h}^{b}:=\left\{(t, s) \in D: t \in\left[b-\varepsilon_{h}, b\right)\right\}$. Let us define $f_{h}^{a} \in \operatorname{Lip}\left(\left[a, a+\varepsilon_{h}\right] ; \mathbb{R}^{2}\right)$ as

$$
f_{h}^{a}(t):=\frac{\phi_{h}\left(a+\varepsilon_{h}, 0\right)-\gamma^{+}(a)}{\varepsilon_{h}}(t-a)+\gamma^{+}(a),
$$

so that its graph is the segment joining $\left(a, \gamma^{+}(a)\right)$ and $\left(a+\varepsilon_{h}, \phi_{h}\left(a+\varepsilon_{h}, 0\right)\right)$. Next, for $(t, s) \in T_{h}^{a}$, set

$$
\Phi_{h}(t, s):= \begin{cases}\left(t, \frac{s}{\sigma^{+}(t)} \gamma^{+}(t)+\frac{\sigma^{+}(t)-s}{\sigma^{+}(t)} f_{h}^{a}(t)\right) & \text { if } s \geq 0, \\ \left(t, \frac{s}{\sigma^{-}(t)} \gamma^{-}(t)+\frac{\sigma^{-}(t)-s}{\sigma^{-}(t)} f_{h}^{a}(t)\right) & \text { if } s<0,\end{cases}
$$

and similarly on $T_{h}^{b}$. Again, Lemma 4.4 and our hypotheses imply

$$
\int_{T_{h}^{a} \cup T_{h}^{b}}\left|\partial_{t} \Phi_{h} \wedge \partial_{s} \Phi_{h}\right| \mathrm{d} t \mathrm{~d} s \stackrel{h \rightarrow+\infty}{\longrightarrow} 0 .
$$

Thus, using (6.6), (6.7) and (6.8) we obtain, for any $h \in \mathbb{N}$,

$$
m\left(D ; \Gamma^{-}, \Gamma^{+}\right) \leq \int_{D}\left|\partial_{t} \Phi_{h} \wedge \partial_{s} \Phi_{h}\right| \mathrm{d} t \mathrm{~d} s \leq m\left(O_{\varepsilon_{h}} ; \Gamma_{h}^{-}, \Gamma_{h}^{+}\right)+\mathcal{O}\left(\varepsilon_{h}\right) .
$$

Passing to the limit as $h \rightarrow+\infty$, (6.4) follows.

For any $d>0$, define $J_{\mathbf{u}}^{d}:=\left\{(t, s) \in \mathbb{R}^{2}: \operatorname{dist}\left((t, s), J_{\mathbf{u}}\right)<d\right\}$. We parametrize the curve $\{s>0\} \cap \partial J_{\mathbf{u}}^{d}$ on the interval $\left(a-\frac{\pi}{2}, b+\frac{\pi}{2}\right)$ by the map $\beta_{d}^{+}$defined by

$$
\beta_{d}^{+}(\theta):= \begin{cases}(a+d \sin (\theta-a), d \cos (\theta-a)) & \text { if } \theta \in(a-\pi / 2, a), \\ (\theta, d) & \text { if } \theta \in[a, b], \\ (b+d \sin (\theta-b), d \cos (\theta-b)) & \text { if } \theta \in(b, b+\pi / 2),\end{cases}
$$

and, similarly, we define the parametrization $\beta_{d}^{-}$for $\{s<0\} \cap \partial J_{\mathbf{u}}^{d}$. We can now introduce the coordinates $(\theta, r)$ in $\mathbb{R}^{2} \backslash\{s=0\}$ such that $(t, s)=\beta_{r}^{+}(\theta)$ if $s>0$, and $(t, s)=\beta_{r}^{-}(\theta)$ if $s<0$.

Proposition 6.3 (Interpolation, II) Let $\Omega^{+}:=\Omega \cap\{s>0\}$, let $\left(\mathbf{u}_{h}\right) \subset \operatorname{Lip}\left(\Omega^{+} ; \mathbb{R}^{2}\right)$, $\mathbf{u} \in \operatorname{Lip}\left(\Omega^{+} ; \mathbb{R}^{2}\right)$, and suppose that $\mathbf{u}_{h} \rightarrow \mathbf{u}$ in $L^{1}\left(\Omega^{+} ; \mathbb{R}^{2}\right)$ as $h \rightarrow+\infty$. Let $\varepsilon_{o}>0$ be fixed so that $\overline{J_{\mathbf{u}}^{\varepsilon_{o}}} \cap\{s>0\} \subset \Omega^{+}$. For any $\varepsilon \in\left(0, \varepsilon_{o}\right]$ we define $\gamma_{h}^{\varepsilon}:=\mathbf{u}_{h} \circ \beta_{\varepsilon}^{+}$and $\gamma^{\varepsilon}:=\mathbf{u} \circ \beta_{\varepsilon}^{+}$. Let us suppose that $\dot{\gamma}^{\varepsilon_{o}}$ exists almost everywhere in $I:=\left(a-\frac{\pi}{2}, b+\frac{\pi}{2}\right)$, and let $\varepsilon_{i} \in\left(0, \varepsilon_{o}\right)$ be such that:

(i) $\left\|\gamma_{h}^{\varepsilon_{i}}-\gamma^{\varepsilon_{i}}\right\|_{L^{\infty}\left(I ; \mathbb{R}^{2}\right)} \rightarrow 0$ as $h \rightarrow+\infty$;

(ii) $\dot{\gamma}_{h}^{\varepsilon_{i}}$ exists almost everywhere in I for any $h \in \mathbb{N}$;

(iii) $\liminf _{h \rightarrow+\infty}\left\|\dot{\gamma}_{h}^{\varepsilon_{i}}\right\|_{L^{1}\left(I ; \mathbb{R}^{2}\right)} \leq M$, where the constant $M$ may depend on $\varepsilon_{i}$. 
Let us define the sequence $\left(\mathbf{v}_{h}\right) \subset \operatorname{Lip}\left(\Omega^{+} ; \mathbb{R}^{2}\right)$ as $\mathbf{v}_{h}:=\mathbf{u}$ on $\Omega^{+} \backslash J_{\mathbf{u}}^{\varepsilon_{o}}, \mathbf{v}_{h}:=\mathbf{u}_{h}$ in $\Omega^{+} \cap J_{\mathbf{u}}^{\varepsilon_{i}}$, and such that its representation in $(\theta, r)$ coordinates in the curvilinear strip $S_{\varepsilon_{i}}^{\varepsilon_{o}}:=\Omega^{+} \cap\left(J_{\mathbf{u}}^{\varepsilon_{o}} \backslash J_{\mathbf{u}}^{\varepsilon_{i}}\right)$ is

$$
\tilde{\mathbf{v}}_{h}(\theta, r):=\frac{\varepsilon_{o}-r}{\varepsilon_{o}-\varepsilon_{i}} \gamma_{h}^{\varepsilon_{i}}(\theta)+\frac{r-\varepsilon_{i}}{\varepsilon_{o}-\varepsilon_{i}} \gamma^{\varepsilon_{o}}(\theta) .
$$

Then

$$
\liminf _{h \rightarrow+\infty} \mathcal{A}\left(\mathbf{v}_{h}, \Omega^{+} \cap\left(J_{\mathbf{u}}^{\varepsilon_{o}} \backslash J_{\mathbf{u}}^{\varepsilon_{i}}\right)\right) \leq C(1+M)\left|\varepsilon_{o}-\varepsilon_{i}\right|,
$$

where $C=C(\operatorname{lip}(\mathbf{u}))$.

Proof The term $\mathcal{A}\left(\mathbf{v}_{h}, S_{\varepsilon_{i}}^{\varepsilon_{o}} \cap\{t \in(a, b)\}\right)$ can be estimated by the right hand side of (6.9) using Proposition 4.9, since in $S_{\varepsilon_{i}}^{\varepsilon_{o}} \cap\{t \in(a, b)\}$ we have $\theta(t, s)=t$ and $r(t, s)=s$.

We prove the estimate for $\mathcal{A}\left(\mathbf{v}_{h}, S_{\varepsilon_{i}}^{\varepsilon_{o}} \cap\{t<a\}\right)$, the computations for $\mathcal{A}\left(\mathbf{v}_{h}, S_{\varepsilon_{i}}^{\varepsilon_{o}} \cap\{t>b\}\right)$ being similar. We have:

$$
\begin{aligned}
\mathcal{A}\left(\mathbf{v}_{h}, S_{\varepsilon_{i}}^{\varepsilon_{o}} \cap\{t<a\}\right) & =\int_{\varepsilon_{i}}^{\varepsilon_{o}} \int_{a-\pi / 2}^{a} \sqrt{r^{2}+\left|\partial_{\theta} \tilde{\mathbf{v}}_{h}\right|^{2}+r^{2}\left|\partial_{r} \tilde{\mathbf{v}}_{h}\right|^{2}+\left(\operatorname{det} \nabla_{\theta, r} \tilde{\mathbf{v}}_{h}\right)^{2}} \mathrm{~d} \theta \mathrm{d} r \\
& \leq C \int_{\varepsilon_{i}}^{\varepsilon_{o}} \int_{a-\pi / 2}^{a}\left[r+\left|\partial_{\theta} \tilde{\mathbf{v}}_{h}\right|+r\left|\partial_{r} \tilde{\mathbf{v}}_{h}\right|+\left|\operatorname{det} \nabla_{\theta, r} \tilde{\mathbf{v}}_{h}\right|\right] \mathrm{d} \theta \mathrm{d} r
\end{aligned}
$$

where $\nabla_{\theta, r}$ denotes the Jacobian with respect to $(\theta, r)$, and $C$ is an absolute positive constant. Again we estimate the right hand side as in the proof of Proposition 4.9 and using our assumptions:

$$
\begin{aligned}
& -\int_{\varepsilon_{i}}^{\varepsilon_{o}} \int_{a-\pi / 2}^{\pi} r \mathrm{~d} r \mathrm{~d} \theta=\pi / 2\left(\varepsilon_{o}^{2}-\varepsilon_{i}^{2}\right), \\
& -\int_{\varepsilon_{i}}^{\varepsilon_{o}} \int_{a-\pi / 2}^{a}\left|\partial_{\theta} \tilde{\mathbf{v}}_{h}\right| \mathrm{d} \theta \mathrm{d} r \leq \int_{\varepsilon_{i}}^{\varepsilon_{o}} \int_{a-\pi / 2}^{a}\left[\left|\dot{\gamma}^{\varepsilon_{o}}\right|+\left|\dot{\gamma}_{h}^{\varepsilon_{i}}\right|\right] \mathrm{d} \theta \mathrm{d} r \leq \pi \varepsilon_{o} \operatorname{lip}(\mathbf{u})\left(\varepsilon_{o}-\varepsilon_{i}\right)+\left(\varepsilon_{o}-\varepsilon_{i}\right) M \\
& -\int_{\varepsilon_{i}}^{\varepsilon_{o}} \int_{a-\pi / 2}^{a} r\left|\partial_{r} \tilde{\mathbf{v}}_{h}\right| \mathrm{d} \theta \mathrm{d} r=\int_{\varepsilon_{i}}^{\varepsilon_{o}} \int_{a-\pi / 2}^{a} r \frac{\left|\gamma^{\varepsilon_{o}}-\gamma_{h}^{\varepsilon_{i}}\right|}{\varepsilon_{o}-\varepsilon_{i}} \mathrm{~d} \theta \mathrm{d} r \\
& \leq \int_{\varepsilon_{i}}^{\varepsilon_{o}} \int_{a-\pi / 2}^{a} r \frac{\left|\gamma^{\varepsilon_{o}}-\gamma^{\varepsilon_{i}}\right|+\left|\gamma^{\varepsilon_{i}}-\gamma_{h}^{\varepsilon_{i}}\right|}{\varepsilon_{o}-\varepsilon_{i}} \mathrm{~d} \theta \mathrm{d} r \leq \frac{\pi}{4} \operatorname{lip}(\mathbf{u})\left(\varepsilon_{o}^{2}-\varepsilon_{i}^{2}\right) \\
& +\frac{\varepsilon_{o}+\varepsilon_{i}}{2} \int_{a-\pi / 2}^{a}\left|\gamma^{\varepsilon_{i}}-\gamma_{h}^{\varepsilon_{i}}\right| \mathrm{d} \theta, \\
& -\int_{\varepsilon_{i}}^{\varepsilon_{o}} \int_{a-\pi / 2}^{a}\left|\operatorname{det} \nabla_{\theta, r} \tilde{\mathbf{v}}_{h}\right| \mathrm{d} \theta \mathrm{d} r \leq 2 \int_{\varepsilon_{i}}^{\varepsilon_{o}} \int_{a-\pi / 2}^{a}\left|\partial_{r} \tilde{\mathbf{v}}_{h}\left\|\partial_{\theta} \tilde{\mathbf{v}}_{h}\left|\mathrm{~d} \theta \mathrm{d} r \leq 2 \int_{a-\pi / 2}^{a}\right| \gamma^{\varepsilon_{o}}-\gamma_{h}^{\varepsilon_{i}}\right\| \dot{\gamma}^{\varepsilon_{o}}+\dot{\gamma}_{h}^{\varepsilon_{i}}\right| \mathrm{d} \theta \\
& \leq \int_{a-\pi / 2}^{a}\left|\dot{\gamma}^{\varepsilon_{o}}\left\|\gamma^{\varepsilon_{o}}-\gamma_{h}^{\varepsilon_{i}}\left|\mathrm{~d} \theta+\int_{a-\pi / 2}^{a}\right| \dot{\gamma}_{h}^{\varepsilon_{i}}\right\| \gamma^{\varepsilon_{o}}-\gamma_{h}^{\varepsilon_{i}}\right| \mathrm{d} \theta \\
& \leq \varepsilon_{o} \operatorname{lip}(\mathbf{u})\left(\operatorname{lip}(\mathbf{u})\left|\varepsilon_{o}-\varepsilon_{i}\right|+|| \gamma^{\varepsilon_{i}}-\gamma_{h}^{\varepsilon_{i}}||_{L^{1}\left(I, \mathbb{R}^{2}\right)}\right)+M\left(\operatorname{lip}(\mathbf{u})\left|\varepsilon_{o}-\varepsilon_{i}\right|+\left\|\gamma^{\varepsilon_{i}}-\gamma_{h}^{\varepsilon_{i}}\right\| \|_{L^{\infty}\left(I ; \mathbb{R}^{2}\right)}\right)
\end{aligned}
$$

Using our assumptions and the previous estimates, we get

$$
\liminf _{h \rightarrow+\infty} \mathcal{A}\left(\mathbf{v}_{h}, S_{\varepsilon_{i}}^{\varepsilon_{o}} \cap\{t<a\}\right) \leq C(1+M)\left|\varepsilon_{o}-\varepsilon_{i}\right|,
$$

where $C=C(\operatorname{lip}(\mathbf{u}))$.

We are now in the position to prove Theorem 6.1. 
Proof Let us suppose first that $\left(\mathbf{u}_{h}\right)$ satisfies (6.2). Let $\left(\varepsilon_{h}\right)$ and $\left(\tilde{\varepsilon}_{h}\right)$ be two infinitesimal sequences of positive numbers such that

$$
N_{h} \subset \subset\left\{(t, s):\left(t, \frac{s}{\tilde{\varepsilon}_{h}}\right) \in O_{\varepsilon_{h}}\right\} \subset \Omega,
$$

where $O_{\varepsilon_{h}}=\left[\left[\sigma_{\varepsilon_{h}}^{-}, \sigma_{\varepsilon_{h}}^{+}\right]\right]$is defined as in Lemma 6.2. Let $\gamma_{h}^{ \pm} \in \operatorname{Lip}\left(\left[a-\varepsilon_{h}, b+\varepsilon_{h}\right] ; \mathbb{R}^{2}\right)$ be defined as

$$
\gamma_{h}^{ \pm}(t):=\mathbf{u}_{h}\left(t, \tilde{\varepsilon}_{h} \sigma_{\varepsilon_{h}}^{ \pm}(t)\right)=\mathbf{u}\left(t, \tilde{\varepsilon}_{h} \sigma_{\varepsilon_{h}}^{ \pm}(t)\right) .
$$

Following the same computation as in Lemma 4.6 we get

$$
\mathcal{A}\left(\mathbf{u}_{h},\left\{(t, s):\left(t, \frac{s}{\tilde{\varepsilon}_{h}}\right) \in O_{\varepsilon_{h}}\right\}\right) \geq m\left(O_{\varepsilon_{h}} ; \Gamma_{h}^{-}, \Gamma_{h}^{+}\right),
$$

where $\Gamma_{h}^{ \pm}:=\operatorname{graph}\left(\gamma_{h}^{ \pm}\right)$. Due to the regularity assumptions on $\mathbf{u}$, the sequences $\left(\gamma_{h}^{ \pm}\right)$satisfy the hypotheses of Lemma 6.2, and thus we can conclude that

$$
\begin{aligned}
\liminf _{h \rightarrow+\infty} \mathcal{A}\left(\mathbf{u}_{h}, \Omega\right) & \geq \int_{\Omega}|\mathcal{M}(\nabla \mathbf{u})| \mathrm{d} t \mathrm{~d} s+\liminf _{h \rightarrow+\infty} m\left(O_{\varepsilon_{h}} ; \Gamma_{h}^{-}, \Gamma_{h}^{+}\right) \\
& \geq \int_{\Omega}|\mathcal{M}(\nabla \mathbf{u})| \mathrm{d} t \mathrm{~d} s+m\left(D ; \Gamma^{-}, \Gamma^{+}\right) .
\end{aligned}
$$

Now, we have to prove that for any sequence $\left(\mathbf{u}_{h}\right)$ converging to $\mathbf{u}$ in $L^{1}\left(\Omega ; \mathbb{R}^{2}\right)$ and uniformly out of $J_{\mathbf{u}}$ we can build a sequence $\left(\mathbf{v}_{h}\right)$ satisfying (6.2) and (6.3). The proof follows along the same lines of Theorem 4.3, where the choice of an infinitesimal sequence $\left(\varepsilon_{k}\right) \subset(0,+\infty)$ satisfying hypotheses (i)-(iii) of Proposition 6.3 is guaranteed by Fatou's lemma applied to the area functional in the $(\theta, r)$ coordinates.

\section{Examples for which $\overline{\mathcal{A}}<\overline{\mathcal{A}}^{\infty}$}

In this section we exhibit some examples of pairs $(\Omega, \mathbf{u})$ satisfying condition II and for which $\overline{\mathcal{A}}(\mathbf{u}, \Omega)<\overline{\mathcal{A}}^{\infty}(\mathbf{u}, \Omega)$. The idea is that, under certain circumstances, sequences converging to $\mathbf{u}$ in $L^{1}\left(\Omega ; \mathbb{R}^{2}\right)$, but not uniformly out of $J_{\mathbf{u}}$, can provide an upper bound lower than the right hand side of (5.1). What is suggested by these examples is that we could extend in some way the jump, adding to $J_{\mathbf{u}}$ a sort of "virtual" jump, and build sequences converging uniformly to $\mathbf{u}$ out of this extension. How choosing these extensions seems not easy. We present different possibilities that confirm the strong non-local behavior of the functional $\overline{\mathcal{A}}(\mathbf{u}, \cdot)$.

\subsection{Virtual jump starting from an endpoint of $J_{\mathbf{u}}$}

In [1, Section 5] the authors study $\overline{\mathcal{A}}\left(\mathbf{u}_{V}, B_{R}\right)$, with $\mathbf{u}_{V}(t, s):=\frac{(t, s)}{|(t, s)|},(t, s) \in \mathbb{R}^{2} \backslash\{0\}$ (the vortex map), providing two different upper bounds. In [1, Lemma 5.2] they bound $\overline{\mathcal{A}}_{s}\left(\mathbf{u}_{V}, B_{R}\right)$ by the measure of the 2-dimensional unit disk, while in [1, Lemma 5.3] by the lateral area of a cylinder, whose height is the distance between the vortex and the boundary of $B_{R}$ (namely, $R$ ), and whose basis is the unit disk.

The idea of [1, Lemma 5.3] is the following. Let us express the map $\mathbf{u}_{V}$ in polar coordinates $(r, \theta)$, without renaming it, i.e., $\mathbf{u}_{V}(r, \theta)=(\cos \theta, \sin \theta)$. Let $\left(\theta_{h}\right)$ and $\left(r_{h}\right)$ be two infinitesimal sequences of positive numbers, and let us define the functions $f_{h}:[-\pi, \pi] \rightarrow \mathbb{R}$, $g_{h}:[0, R) \rightarrow[0,+\infty)$ as 
$f_{h}(\theta):=\left\{\begin{array}{ll}-\frac{\pi-\theta_{h}}{\theta_{h}}(\theta+\pi) & \text { if } \theta \in\left[-\pi,-\pi+\theta_{h}\right), \\ \theta & \text { if } \theta \in\left[-\pi+\theta_{h}, \pi-\theta_{h}\right), \\ -\frac{\pi-\theta_{h}}{\theta_{h}}(\theta-\pi) & \text { if } \theta \in\left[\pi-\theta_{h}, \pi\right),\end{array} \quad g_{h}(r):= \begin{cases}\frac{r}{r_{h}} & \text { if } r \in\left(0, r_{h}\right], \\ 1 & \text { if } r \in\left(r_{h}, R\right) .\end{cases}\right.$

Then, the sequence $\left(\mathbf{u}_{h}\right) \subset \operatorname{Lip}\left(B_{R} ; \mathbb{R}^{2}\right)$ defined by

$$
\mathbf{u}_{h}(r, \theta):=\left(\cos \left(g_{h}(r) f_{h}(\theta)\right), \sin \left(g_{h}(r) f_{h}(\theta)\right)\right)
$$

converges to $\mathbf{u}_{V}$ in $L^{1}\left(B_{R} ; \mathbb{R}^{2}\right)$ and $\lim _{h \rightarrow+\infty} \mathcal{A}\left(\mathbf{u}_{h}, B_{R}\right) \leq \int_{B_{R}}\left|\mathcal{M}\left(\nabla \mathbf{u}_{V}\right)\right| \mathrm{d} t \mathrm{~d} s+2 \pi R .{ }^{9}$

We adapt the procedure of [1, Lemma 5.3] when $\Omega$ and $\mathbf{u}$ satisfy condition II, and we build a sequence $\left(\mathbf{u}_{h}\right) \subset \operatorname{Lip}\left(\mathrm{R} ; \mathbb{R}^{2}\right)$ converging to $\mathbf{u}$ in $L^{1}\left(\Omega ; \mathbb{R}^{2}\right)$ and uniformly out of a curve containing $J_{\mathbf{u}}$ and having an endpoint on $\partial \Omega$. In this case the virtual jump connects one endpoint of $J_{\mathbf{u}}$ and $\partial \Omega$. The singular contribution

$$
\lim _{h \rightarrow+\infty} \mathcal{A}\left(\mathbf{u}_{h}, \Omega\right)-\int_{\Omega}|\mathcal{M}(\nabla \mathbf{u})| \mathrm{d} t \mathrm{~d} s
$$

can be interpreted as the area of a suitable semicartesian parametrization (Remark 7.2) with non-empty partially free boundary and, under certain circumstances, it is lower than $m\left(D ; \Gamma^{-}[\mathbf{u}], \Gamma^{+}[\mathbf{u}]\right)$, see inequality (7.10); compare also with (1.6).

Proposition 7.1 Let $\Omega$ and $\mathbf{u}$ satisfy condition II, with the further conditions that $\Omega \cap\{s=$ $0\}=\left(a_{1}, b+\delta\right) \times\{0\}$ for some $a_{1}<a$ and $\delta>0$, and

$$
\mathbf{u} \in \mathcal{C}^{1}\left(\overline{\Omega \cap\{s>0\}} ; \mathbb{R}^{2}\right) \cap \mathcal{C}^{1}\left(\overline{\Omega \cap\{s<0\}} ; \mathbb{R}^{2}\right) .
$$

Then there exists a sequence $\left(\mathbf{u}_{h}\right) \subset \operatorname{Lip}\left(\Omega ; \mathbb{R}^{2}\right)$ converging to $\mathbf{u}$ in $L^{1}\left(\Omega ; \mathbb{R}^{2}\right)$ such that

$$
\begin{aligned}
& \lim _{h \rightarrow+\infty} \mathcal{A}\left(\mathbf{u}_{h}, \Omega\right) \leq \int_{\Omega}|\mathcal{M}(\nabla \mathbf{u})| d t d s \\
& +(b+\delta-a)\left\{\int_{a}^{b}\left[\left|\dot{\gamma}^{-}[\mathbf{u}]\right|+\left|\dot{\gamma}^{+}[\mathbf{u}]\right|\right] d t+2 \int_{b}^{b+\delta}\left|\partial_{t} \mathbf{u}(t, 0)\right| d t\right\} .
\end{aligned}
$$

Hence

$$
\overline{\mathcal{A}}_{s}(\mathbf{u}, \Omega) \leq(b+\delta-a)\left\{\int_{a}^{b}\left[\left|\dot{\gamma}^{-}[\mathbf{u}]\right|+\left|\dot{\gamma}^{+}[\mathbf{u}]\right|\right] d t+2 \int_{b}^{b+\delta}\left|\partial_{t} \mathbf{u}(t, 0)\right| d t\right\} .
$$

Proof Given an infinitesimal sequence $\left(\varepsilon_{h}\right)$ of positive numbers, define (Fig. 5)

$$
C_{\varepsilon_{h}}:=\left\{(t, s) \in \Omega: t>a,|s|<\varepsilon_{h}(t-a)\right\},
$$

and $r_{\varepsilon_{h}}: \Omega \rightarrow \Omega \backslash C_{\varepsilon_{h}}$ as

$$
r_{\varepsilon_{h}}(t, s):= \begin{cases}(t, s) & (t, s) \in \Omega \backslash C_{\varepsilon_{h}} \\ \left(\frac{s}{\varepsilon_{h}}+a, s\right) & (t, s) \in C_{\varepsilon_{h}}, s \geq 0 \\ \left(-\frac{s}{\varepsilon_{h}}+a, s\right) & (t, s) \in C_{\varepsilon_{h}}, s<0\end{cases}
$$

\footnotetext{
9 It is possible to improve the estimate of [1, Lemma 5.3], obtaining, as singular contribution, the area of a catenoid in place of the lateral area of a cylinder. Let us suppose $R>0$ to be so small that there exists a catenary $c:(0, R) \rightarrow(0,+\infty)$ such that $c(0)=c(R)=1$. Then, taken an infinitesimal sequence $\left(\omega_{h}\right)$ with $\omega_{h}>\theta_{h}$ and $\omega_{h} / \theta_{h} \rightarrow 1$ as $h \rightarrow+\infty$, we define $\rho_{h} \in \operatorname{Lip}\left(B_{R}\right)$ such that $\rho_{h}(r, \theta):=c(r)$ if $\theta \in\left[-\pi,-\pi+\theta_{h}\right) \cup\left[\pi-\theta_{h}, \theta\right)$ and $\rho_{h}(r, \theta):=1$ if $\theta \in\left(-\pi+\omega_{h}, \pi-\omega_{h}\right)$. Then the sequence $\left(\mathbf{u}_{h}\right)$ defined by $\mathbf{u}_{h}(r, \theta):=\rho_{h}(r, \theta)\left(\cos \left(g_{h}(r) f_{h}(\theta)\right), \sin \left(g_{h}(r) f_{h}(\theta)\right)\right)$ provides the desired estimate.
} 


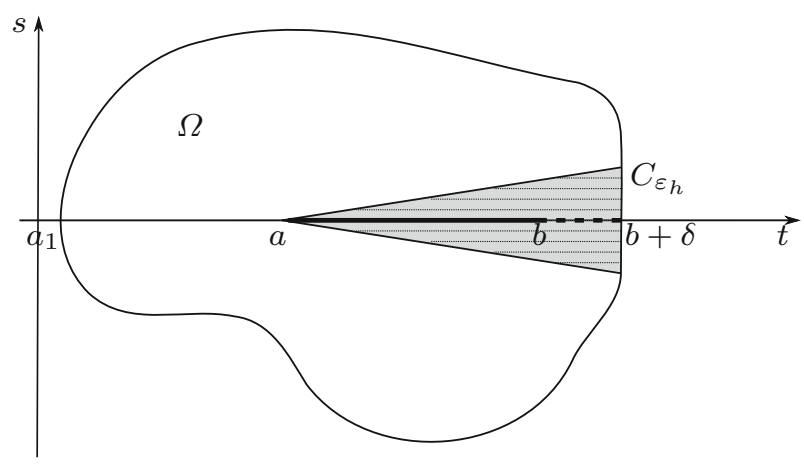

Fig. 5 The set $\Omega$ and, in gray, the triangle $C_{\varepsilon_{h}}$ built in Proposition 7.1. The map $\mathbf{u}_{h}$ defined in (7.6) is constant on the horizontal segments in $C_{\varepsilon_{h}}$. The sequence $\left(\mathbf{u}_{h}\right)$ converges to $\mathbf{u}$ in $L^{1}\left(\Omega ; \mathbb{R}^{2}\right)$ and uniformly out of the segment $J_{\mathrm{ext}}=(a, b+\delta) \times\{0\}$, union of $J_{\mathbf{u}}$ (the bold segment) and of the virtual jump $[b, b+\delta] \times\{0\}$, represented by a bold dotted line

that is the retraction mapping each point $(t, s) \in C_{\varepsilon_{h}}$ into the point of $\partial C_{\varepsilon_{h}} \cap \Omega$ having $s$ as second coordinate.

Let us define the sequence $\left(\mathbf{u}_{h}\right) \subset \operatorname{Lip}\left(\Omega ; \mathbb{R}^{2}\right)$ as

$$
\mathbf{u}_{h}(t, s):=\mathbf{u}\left(r_{\varepsilon_{h}}(t, s)\right), \quad(t, s) \in \Omega .
$$

We observe that $\left(\mathbf{u}_{h}\right)$ converges to $\mathbf{u}$ in $L^{1}\left(\Omega ; \mathbb{R}^{2}\right)$ but not uniformly out of $J_{\mathbf{u}}$, as $h \rightarrow+\infty$. Incidentally, we notice that $\left(\mathbf{u}_{h}\right)$ converges to $\mathbf{u}$ uniformly out of a suitable "extension" of the jump, $J_{\text {ext }}:=(a, b+\delta) \times\{0\}$.

Denoting by $\partial_{1} \mathbf{u}$ and $\partial_{2} \mathbf{u}$ the derivative with respect to the first and second variable of $\mathbf{u}$, let us compute the area of the graph of $\mathbf{u}_{h}$ on $C_{\varepsilon_{h}} \cap\{s \geq 0\}^{10}$ :

$$
\begin{aligned}
\mathcal{A} & \left(\mathbf{u}_{h}, C_{\varepsilon_{h}} \cap\{s \geq 0\}\right) \\
& =\int_{a}^{b+\delta} \int_{0}^{\varepsilon_{h}(t-a)} \sqrt{1+\frac{1}{\varepsilon_{h}^{2}}\left|\partial_{1} \mathbf{u}\left(\frac{s}{\varepsilon_{h}}+a, s\right)\right|^{2}+\left|\partial_{2} \mathbf{u}\left(\frac{s}{\varepsilon_{h}}+a, s\right)\right|^{2}} \mathrm{~d} s \mathrm{~d} t \\
& =\int_{a}^{b+\delta} \int_{0}^{\varepsilon_{h}(t-a)} \frac{1}{\varepsilon_{h}} \sqrt{\left|\partial_{1} \mathbf{u}\left(\frac{s}{\varepsilon_{h}}+a, s\right)\right|^{2}+\mathcal{O}\left(\varepsilon_{h}^{2}\right)} \mathrm{d} s \mathrm{~d} t \\
& =\int_{a}^{b+\delta} \int_{a}^{t} \sqrt{\left|\partial_{1} \mathbf{u}\left(\tau, \varepsilon_{h}(\tau-a)\right)\right|^{2}+\mathcal{O}\left(\varepsilon_{h}^{2}\right)} \mathrm{d} \tau \mathrm{d} t \\
& \leq(b+\delta-a) \int_{a}^{b+\delta} \sqrt{\left|\partial_{1} \mathbf{u}\left(\tau, \varepsilon_{h}(\tau-a)\right)\right|^{2}+\mathcal{O}\left(\varepsilon_{h}^{2}\right)} \mathrm{d} \tau .
\end{aligned}
$$

Similarly

$$
\mathcal{A}\left(\mathbf{u}_{h}, C_{\varepsilon_{h}} \cap\{s<0\}\right) \leq(b+\delta-a) \int_{a}^{b+\delta} \sqrt{\left|\partial_{1} \mathbf{u}\left(\tau,-\varepsilon_{h}(\tau-a)\right)\right|^{2}+\mathcal{O}\left(\varepsilon_{h}^{2}\right)} \mathrm{d} \tau .
$$

Thus, noticing that $\left|\partial_{1} \mathbf{u}\left(\tau, \pm \varepsilon_{h}(\tau-a)\right)\right|$ are uniformly bounded and that $\partial_{1} \mathbf{u}\left(\tau, \pm \varepsilon_{h}(\tau-\right.$ $a)) \rightarrow \dot{\gamma}^{ \pm}[\mathbf{u}](\tau)$ pointwise for $\tau \in(a, b)$, and $\partial_{1} \mathbf{u}\left(\tau, \pm \varepsilon_{h}(\tau-a)\right)=\partial_{1} \mathbf{u}(\tau, 0)$ in $(b, b+\delta)$, possibly passing to a subsequence we get (7.2)

10 The computation is done supposing that the triangle $\left\{(t, s): t \in(a, b+\delta),|t| \leq \varepsilon_{h}(t-a)\right\}$ is contained in $\Omega$, but it can be easily arranged to a more general situation. 
Remark 7.2 (Semicartesian interpretation)The right hand side of (7.3) can be interpreted as the area of the semicartesian parametrization built in Example 2.12, with $b+\delta$ in place of $b$ and with $\mathrm{C}:=\gamma([0,2(b+\delta-a)])$, where $\gamma:[0,2(b+\delta-a)] \rightarrow \mathbb{R}^{2}$ is defined as follows:

$$
\gamma(t):= \begin{cases}\mathbf{u}(b+\delta-t, 0) & \text { if } t \in(0, \delta), \\ \gamma^{+}(b+\delta-t) & \text { if } t \in(\delta, b+\delta-a), \\ \gamma^{-}(t+2 a-b-\delta) & \text { if } t \in(b+\delta-a, 2(b-a)+\delta), \\ \mathbf{u}(t+2 a-b-\delta, 0) & \text { if } t \in(2(b-a)+\delta, 2(b-a+\delta)) .\end{cases}
$$

We notice also that this construction can be done even if $\Omega$ and $\mathbf{u}$ satisfy condition I and $\gamma^{-}(a)=\gamma^{+}(a)$ (or, symmetrically, if $\gamma^{-}(b)=\gamma^{+}(b)$ ). In this case the sequence $\left(\mathbf{u}_{h}\right)$ built in Proposition 7.1 would converge to $\mathbf{u}$ in $L^{1}\left(\mathrm{R} ; \mathbb{R}^{2}\right)$ and uniformly out of $J_{\mathbf{u}}$, and $\lim _{h \rightarrow+\infty} \mathcal{A}\left(\mathbf{u}_{h}, \mathrm{R}\right)-\int_{\mathrm{R}}|\mathcal{M}(\nabla \mathbf{u})| \mathrm{d} t \mathrm{~d} s \geq \overline{\mathcal{A}}_{s}^{\infty}(\mathbf{u}, \mathrm{R})=m\left(\mathrm{R} ; \Gamma^{-}[\mathbf{u}], \Gamma^{+}[\mathbf{u}]\right)$.

Remark 7.3 (Relations with the vortex) Even if Proposition 7.1 has been inspired by the construction in [1, Lemma 5.3], it is worth to underline some important differences. When $\Omega=B_{R}$ and $\mathbf{u}=\mathbf{u}_{V}$, we could interpret the origin as a "collapsed" jump, and the radius $\{(t, 0): t \in(-R, 0)\}$ as the virtual jump. Differently from the case where $\Omega$ and $\mathbf{u}$ satisfy condition II, the vortex map $\mathbf{u}_{V}$ (which belongs to $W^{1, p}\left(B_{R} ; \mathbb{S}^{1}\right)$ for any $p \in[1,2)$ ) does not admit any limit as $(t, s) \rightarrow(0,0),(t, s) \in B_{R} \backslash\{(t, 0): t \in(-R, 0)\}$. Intuitively if we would interpret the bound $\overline{\mathcal{A}}_{s}\left(\mathbf{u}_{V}, B_{R}\right) \leq 2 \pi R$ in terms of area of semicartesian parametrizations, this lack of continuity of $\mathbf{u}_{V}$ at $(0,0)$ would force us to consider only surfaces having as trace on the plane $\{t=0\} \times \mathbb{R}_{(\xi, \eta)}^{2}$ the unit circumference, covered with the right orientation. We also notice that in [1, Lemma 5.3] the sequence $\left(\mathbf{u}_{h}\right)$ is defined as the composition of $\mathbf{u}_{V}$ and a suitable retraction from $B_{R}$ to $B_{R} \backslash C_{\varepsilon_{h}}$, where $\left(C_{\varepsilon_{h}}\right)$ is a decreasing sequence of circular sectors containing $\{(t, 0): t \in(-R, 0)\}$ and converging to it. In [1] the image of $C_{\varepsilon_{h}}$ through this retraction covers the whole of $B_{R} \backslash C_{\varepsilon_{h}}$, while in our case (for $C_{\varepsilon_{h}}$ now as in (7.4)) it is contained in $\partial C_{\varepsilon_{h}}$, see (7.5); this difference is due to the fact that, contrary to our case, the trace of the vortex map on the boundary of each circular sector is not continuous.

In the next example, we exhibit a map $\mathbf{u}$ for which the sequence built in Proposition 7.1 provides an upper bound that is lower than $m\left(D ; \Gamma^{-}, \Gamma^{+}\right)$. This example, coupled with Theorem 1.4 , shows that $\overline{\mathcal{A}}(\mathbf{u}, \Omega)<\overline{\mathcal{A}}^{\infty}(\mathbf{u}, \Omega)$.

Example 7.4 (Difference in the two relaxations) Let $\Omega$ be as in Proposition 7.1, and define ${ }^{11}$

$\mathbf{u}(t, s):= \begin{cases}(1,0) & \text { in }\{(t, s) \in \Omega: s \geq 0\}, \\ (1,0) & \text { in }\{(t, s) \in \Omega: s<0, t<a \text { or } t>b\}, \\ \rho(\cos (\theta(t)), \sin (\theta(t)))+(1-\rho, 0) & \text { in }\{(t, s) \in \Omega: s<0, a \leq t \leq b\},\end{cases}$

where

$$
\rho>2(b+\delta-a),
$$

and $\theta:[a, b] \rightarrow[0,2 \pi]$ is defined in (2.11). We observe that $\gamma^{+}$is constant, $\gamma^{-}$covers once the circumference centered at $(1-\rho, 0)$ with radius $\rho$, and $\partial_{t} \mathbf{u}(t, 0)=0$ for $t \in(b, b+\delta)$. In this case, formula (7.3) reads as

$$
\overline{\mathcal{A}}_{s}(\mathbf{u}, \Omega) \leq(b+\delta-a) \int_{a}^{b}\left|\dot{\gamma}^{-}\right| \mathrm{d} t=(b+\delta-a) 2 \pi \rho .
$$

11 The map u defined in this way does not satisfy (7.1); anyway the fact that it does not depend on $s$ in $\Omega \cap\{s>0\}$ and in $\Omega \cap\{s<0\}$ allows to obtain (7.2). 


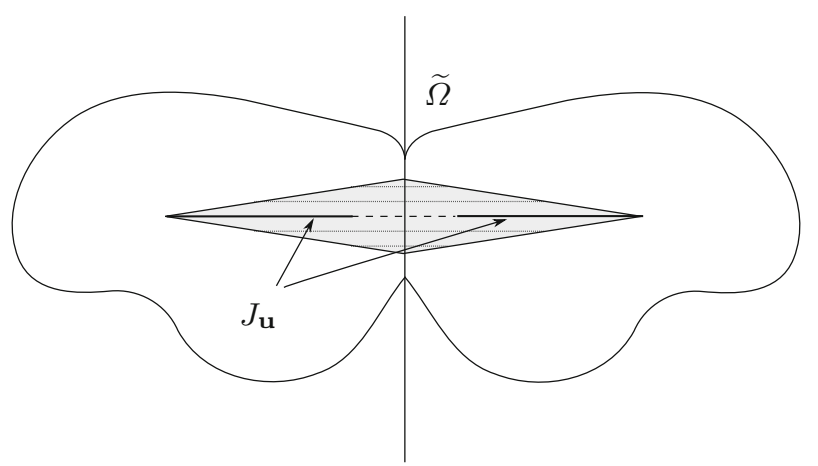

Fig. 6 Remark 7.5. The set $\widetilde{\Omega}$ is built by reflecting, with respect to the vertical axis in the figure, the set $\Omega$ considered in Proposition 7.1. The map $\mathbf{u}: \widetilde{\Omega} \rightarrow \mathbb{R}^{2}$ is defined again by reflecting the map in (7.7), so that $J_{\mathbf{u}}$ has two connected components. It is then possible to build a sequence $\left(\mathbf{u}_{h}\right) \subset \operatorname{Lip}\left(\Omega ; \mathbb{R}^{2}\right)$, again by reflection, converging to $\mathbf{u}$ in $L^{1}\left(\widetilde{\Omega} ; \mathbb{R}^{2}\right)$ and uniformly out of the curve composed of $J_{\mathbf{u}}$ and of the bold dotted segment (the virtual jump) joining the two components of the jump. This sequence provides an upper bound for $\overline{\mathcal{A}}(\mathbf{u}, \widetilde{\Omega})$ lower than the one obtained by any sequence converging to u uniformly out of $J_{\mathbf{u}}$

On the other hand, we have already observed in Example 2.13 that $a(\Gamma) \geq \pi \rho^{2}$. Thus, from (7.8) we obtain that $a(\Gamma)$ is strictly greater than the right hand side of (7.9). Since in general $m\left(D ; \Gamma^{-}, \Gamma^{+}\right) \geq a(\Gamma)$, see Remark 2.11, we have, using Theorem 1.4,

$$
\overline{\mathcal{A}}_{s}(\mathbf{u}, \Omega)<m\left(D ; \Gamma^{-}[\mathbf{u}], \Gamma^{+}[\mathbf{u}]\right)=\overline{\mathcal{A}}_{s}^{\infty}(\mathbf{u}, \Omega) .
$$

Remark 7.5 (Joining two components of $J_{\mathbf{u}}$ ) Example 7.4 suggests that, if the jump set of a discontinuous map $\mathbf{u}: \Omega \rightarrow \mathbb{R}^{2}$ is not connected, it could be convenient (as far as only the $L^{1}\left(\Omega ; \mathbb{R}^{2}\right)$-convergence is involved) considering sequences $\left(\mathbf{u}_{h}\right) \subset \operatorname{Lip}\left(\Omega ; \mathbb{R}^{2}\right)$ converging to $\mathbf{u}$ in $L^{1}\left(\Omega ; \mathbb{R}^{2}\right)$ and uniformly out of a connected curve containing $J_{\mathbf{u}}$. Let $\Omega$ be as in Example 7.4 and let $\widetilde{\Omega}$ be the union of $\Omega$ and of its symmetrized with respect to the axis $\{t=c\}$, for some $c \in(b, b+\delta)$, see Fig. 6. Let us define $\mathbf{u}$ and $\left(\mathbf{u}_{h}\right)$ in $\widetilde{\Omega}$ as in Example 7.4 for $(t, s) \in \Omega$, and by reflection elsewhere in $\widetilde{\Omega}$. Then

$$
\lim _{h \rightarrow+\infty} \mathcal{A}\left(\mathbf{u}_{h}, \widetilde{\Omega}\right)-\int_{\widetilde{\Omega}}|\mathcal{M}(\nabla \mathbf{u})| \mathrm{d} t \mathrm{~d} s \leq 4 \pi \rho(c-a) .
$$

If $\rho>2(c-a)$, the right hand side of $(7.11)$ is smaller than $2 m\left(D ; \Gamma^{-}, \Gamma^{+}\right)$(where $\Gamma^{ \pm}$are the graph of the traces of $\mathbf{u}$ on $(a, b) \times\{0\})$, that would be the bound obtained reasoning as in Proposition 5.1 in distinct neighborhoods of the two connected components of $J_{\mathbf{u}}$. Indeed, as observed in Example 7.4, $m\left(D ; \Gamma^{-}, \Gamma^{+}\right) \geq a(\Gamma) \geq \pi \rho^{2}>4 \pi \rho(c-a)$. Moreover, if $J_{\mathbf{u}}$ is far from $\partial \widetilde{\Omega}$, the right hand side of (7.11) is also smaller than the upper bound obtained by connecting each component of $J_{\mathbf{u}}$ with $\partial \widetilde{\Omega}$, using the construction in Proposition 7.1.

\subsection{Virtual jump starting from an interior point of $J_{\mathrm{u}}$}

In this section we show, for a particular pair $(\Omega, \mathbf{u})$ satisfying condition II, how to build a sequence $\left(\mathbf{u}_{h}\right)$ of maps converging to $\mathbf{u}$ in $L^{1}\left(\Omega ; \mathbb{R}^{2}\right)$ and uniformly out of $J_{\text {ext }}$, union of $J_{\mathbf{u}}$ and a virtual jump connecting an interior point of $J_{\mathbf{u}}$ and $\partial \Omega$. Such phenomena indicate that the characterization of $\overline{\mathcal{A}}_{s}$ is involved, and justify, once more, the study of the functional $\overline{\mathcal{A}}_{s}^{\infty}$. 
Let the bounded connected open set $\Omega$ be such that $\{(\bar{t}, s): s>0\} \cap \Omega=\{\bar{t}\} \times(0, \delta)$ for some $\bar{t} \in(a, b)$. Without loss of generality we can suppose $\bar{t}=0$ and $a=-b$; we take $\Omega:=(-L, L) \times(-1, \delta)$, for $L>b>0$ and $\delta \in(0,1)$, see Fig. 7. For $\rho>2(\delta+b)$ we define $\mathbf{u}: \Omega \rightarrow \mathbb{R}^{2}$ (similarly to Example 7.4) as

$$
\mathbf{u}(t, s):= \begin{cases}\rho\left(\cos \left(\frac{\pi}{b} t+\pi\right), \sin \left(\frac{\pi}{b} t+\pi\right)\right)+(1-\rho, 0) & \text { if }|t| \leq b, s<0 \\ (1,0) & \text { otherwise. }\end{cases}
$$

We want to build a sequence $\left(\mathbf{u}_{h}\right) \subset \operatorname{Lip}\left(\Omega ; \mathbb{R}^{2}\right)$ converging to $\mathbf{u}$ in $L^{1}\left(\Omega ; \mathbb{R}^{2}\right)$ and uniformly out of

$$
J_{\mathrm{ext}}:=J_{\mathbf{u}} \cup(\{0\} \times(0, \delta)),
$$

where $J_{\text {ext }} \backslash J_{\mathbf{u}}=\{0\} \times(0, \delta)$ takes the role of the virtual jump (see (7.13) below) and such that

$$
\lim _{h \rightarrow+\infty} \mathcal{A}\left(\mathbf{u}_{h}, \Omega\right)=\int_{\Omega}|\mathcal{M}(\nabla \mathbf{u})| \mathrm{d} t \mathrm{~d} s+2 \pi \rho(\delta+b) .
$$

To this purpose, for $\varepsilon \in(0, \min \{\delta / 2, b / 2\})$ set (see Fig. 7)

$$
\begin{aligned}
\mathbb{T}_{\varepsilon} & :=\left\{(t, s) \in \Omega:|t|<b, s>0, \operatorname{dist}\left((t, s), J_{\text {ext }}\right)<\varepsilon\right\}, \\
\mathbb{T}_{\varepsilon}^{-} & :=\mathbb{T}_{\varepsilon} \cap\{t<0\}, \quad \mathbb{T}_{\varepsilon}^{+}:=\mathbb{T}_{\varepsilon} \cap\{t>0\} .
\end{aligned}
$$

The definitions will be given on $\mathbb{T}_{\varepsilon}^{+}$and next extended on the whole of $\mathbb{T}_{\varepsilon}$ by reflection with respect to the $s$-axis. Let us parametrize the (closure of the) curve $\Omega \cap\{t>0\} \cap \partial \mathbb{T}_{\varepsilon}$ by the arc-length parametrization $\lambda_{\varepsilon} \in \operatorname{Lip}\left([0, \delta+2 b-\varepsilon] ; \mathbb{R}^{2}\right)$ with $\lambda_{\varepsilon}(0)=(\varepsilon, \delta)$ and $\lambda_{\varepsilon}(\delta+2 b-\varepsilon)=(0,0)$. Let us also parametrize the (closure of the) set $J_{\text {ext }}^{+}:=(\{0\} \times[0, \delta)) \cup$ $([0, b) \times\{0\})$ by the arc-length parametrization $\tilde{\alpha} \in \operatorname{Lip}\left([0, \delta+b] ; \mathbb{R}^{2}\right)$, with $\tilde{\alpha}(0)=(0, \delta)$ and $\tilde{\alpha}(b+\delta)=(b, 0)$.

On $\mathbb{T}_{\varepsilon}^{+}$we consider the change of coordinates $(p, d): \mathbb{T}_{\varepsilon}^{+} \rightarrow S_{\varepsilon}^{+}$defined as follows ${ }^{12}$ :

- $p(t, s) \in(0, \delta+b)$ is the image through $\tilde{\alpha}^{-1}$ of the endpoint on $J_{\mathrm{ext}}^{+}$of the segment represented in Fig. 7 passing through $(t, s)$;

$-d(t, s)$ is the distance between $(t, s)$ and $\tilde{\alpha}(p(t, s))$.

The set $S_{\varepsilon}^{+}$is therefore $\left\{(p, d) \in \mathbb{R}^{2}: p \in(0, \delta+b), d \in\left(0, d_{\max }(p)\right)\right\}$, where

$$
d_{\max }(p)= \begin{cases}\varepsilon & \text { if } p \in(0, \delta-\varepsilon], \\ \sqrt{\varepsilon^{2}+(p-\delta+\varepsilon)^{2}} & \text { if } p \in(\delta-\varepsilon, \delta], \\ \sqrt{\varepsilon^{2}+(-p+\delta+\varepsilon)^{2}} & \text { if } p \in(\delta, \delta+\varepsilon], \\ \varepsilon & \text { if } p \in(\delta+\varepsilon, \delta+b) .\end{cases}
$$

Now, we define a function $\ell_{\varepsilon}: S_{\varepsilon}^{+} \rightarrow(0, \delta+2 b-\varepsilon)$, linear on each segment $\{p\} \times$ $\left(0, d_{\max }(p)\right)$ and such that:

for $p \in(0, \delta-\varepsilon], \ell_{\varepsilon}(p, 0)=\delta+2 b-\varepsilon$ and $\ell_{\varepsilon}\left(p, d_{\max }(p)\right)=p$;

for $p \in(\delta-\varepsilon, \delta], \ell_{\varepsilon}(p, 0)=\delta+2 b-\varepsilon$ and $\ell_{\varepsilon}\left(p, d_{\max }(p)\right)=\delta-\varepsilon$;

for $p \in(\delta, \delta+\varepsilon], \ell_{\varepsilon}(p, 0)=2 \delta+2 b-\varepsilon-p$ and $\ell_{\varepsilon}\left(p, d_{\max }(p)\right)=\delta-\varepsilon$;

for $p \in(\delta+\varepsilon, \delta+b), \ell_{\varepsilon}(p, 0)=2 \delta+2 b-\varepsilon-p$ and $\ell_{\varepsilon}\left(p, d_{\max }(p)\right)=p-2 \varepsilon$.

Thus we can define the retraction $r_{\varepsilon}=\left(r_{\varepsilon, 1}, r_{\varepsilon, 1}\right): \Omega \rightarrow \Omega \backslash \mathbb{T}_{\varepsilon}$ as:

12 Even if not explicitly written, the coordinates $(p, d)$ depend on $\varepsilon$. 


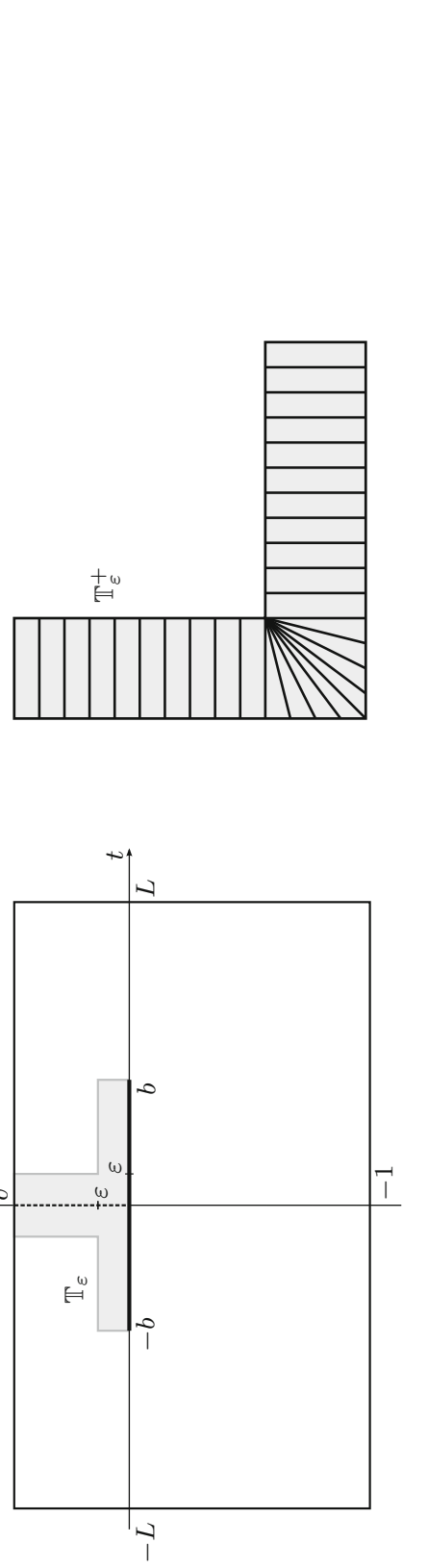

$\Xi$ خै

窟诺

क力 0

IF

$\approx$ bD

$\stackrel{\omega}{\ominus} \frac{\overline{0}}{0}$

$\overline{0} \div$

o

类

둘

$\therefore$

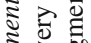

密它

$\stackrel{\infty}{2}=$

ธี ซ

$\approx$ :

㯊

존잉

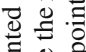

可

눙

원

27

os

e+w

$\times$

$\tilde{0}: \Xi$

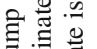

吾

픙

青宫:

\& 0

$\circ=$

క

으를

$\times$ 鹃

$\checkmark$ 过

0 i

I 홍 를

స

을

矛包

ะิ)

종

ริ)

$\Xi+\infty \circ$

䒕

$c: 3$

ป 풘

w 0

Ð

농

ज़

范至

s :

ฮ

$\Xi 20$

几 혼

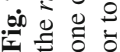


$-r_{\varepsilon}:=\mathrm{id}$ on $\Omega \backslash \mathbb{T}_{\varepsilon}$

$-r_{\varepsilon}(t, s):=\lambda_{\varepsilon}\left(\ell_{\varepsilon}(p(t, s), d(t, s))\right)$ if $(t, s) \in \mathbb{T}_{\varepsilon}^{+}$;

$-r_{\varepsilon}(0, s):=(0,0)$ if $s \in[0, \delta)$;

$-r_{\varepsilon}(t, s):=\left(-r_{\varepsilon, 1}(-t, s), r_{\varepsilon, 2}(-t, s)\right)$ if $(t, s) \in \mathbb{T}_{\varepsilon}^{-}$.

In words, on $\mathbb{T}_{\varepsilon}^{+}$the map $r_{\varepsilon}$ sends each segment in Fig. 7 into $\partial \mathbb{T}_{\varepsilon} \cap\{t>0\}$ in such a way that:

- if both the endpoints of the segment lie on $\partial \mathbb{T}_{\varepsilon} \cap\{t>0\}$ (namely, the vertical segments and the oblique segments below the diagonal), the image of the segment is the portion of $\partial \mathbb{T}_{\varepsilon} \cap\{t>0\}$ bounded by the two endpoints;

- if only one of the endpoints of the segment lies on $\partial \mathbb{T}_{\varepsilon} \cap\{t>0\}$, the image of the segment is the portion of $\partial \mathbb{T}_{\varepsilon} \cap\{t>0\}$ bounded by that endpoint and $(0,0)$.

Let $A_{\varepsilon}:=r_{\varepsilon}^{-1}\left(J_{\mathbf{u}}\right) \subset T_{\varepsilon}$; the image of $A_{\varepsilon} \cap \mathbb{T}_{\varepsilon}^{+}$through the coordinate change $(p, d)$ is the subset of $S_{\varepsilon}^{+}$given by $\left\{(p, d): p \in(0, \delta+b), d \in\left(0, d_{J_{\mathbf{u}}}(p)\right]\right\}$, where

$$
d_{J_{\mathbf{u}}}(p):= \begin{cases}\frac{b}{\delta+2 b-\varepsilon-p} \varepsilon & \text { if } p \in(0, \delta-\varepsilon], \\ \frac{d_{\max }(p)}{2} & \text { if } p \in(\delta-\varepsilon, 0], \\ \frac{\delta+b-p}{\delta+2 b-p} d_{\max }(p) & \text { if } p \in(0, \delta+\varepsilon], \\ \frac{\delta+b-p}{2 \delta+2 b-2 p+\varepsilon} \varepsilon & \text { if } p \in(\delta+\varepsilon, \delta+b) .\end{cases}
$$

We are now in the position to define $\left(\mathbf{u}_{h}\right) \subset \operatorname{Lip}\left(\Omega ; \mathbb{R}^{2}\right)$ as

$$
\mathbf{u}_{h}(t, s):= \begin{cases}\mathbf{u}\left(r_{\varepsilon_{h}}(t, s)\right) & \text { if }(t, s) \in \Omega \backslash A_{\varepsilon_{h}}, \\ \gamma^{-}[\mathbf{u}]\left(r_{\varepsilon_{h}, 1}(t, s)\right) & \text { if }(t, s) \in A_{\varepsilon_{h}},\end{cases}
$$

where $\left(\varepsilon_{h}\right) \subset(0, \min \{\delta / 2, b / 2\})$ is an infinitesimal sequence.

Since $\partial_{2} \mathbf{u}=0$ almost everywhere, ${ }^{13}$ we have

$$
\mathcal{A}\left(\mathbf{u}_{h}, \Omega\right)=\int_{\Omega} \sqrt{1+\left|\partial_{1} \mathbf{u}\left(r_{\varepsilon_{h}}\right)\right|^{2}\left(\left(\partial_{t} r_{\varepsilon_{h}}\right)^{2}+\left(\partial_{s} r_{\varepsilon_{h}}\right)^{2}\right)} \mathrm{d} t \mathrm{~d} s .
$$

We observe also that $r_{\varepsilon_{h}}\left(\mathbb{T}_{\varepsilon_{h}} \backslash A_{\varepsilon_{h}}\right) \subseteq \Omega \cap\left(\partial \mathbb{T}_{\varepsilon_{h}} \backslash J_{\mathbf{u}}\right)$, where also $\partial_{1} \mathbf{u}=0$.

Let us compute the area of the graph of the map $\mathbf{u}_{h}$ on $\mathbb{T}_{\varepsilon_{h}}^{+} \cap\left\{s>\varepsilon_{h}\right\}$, on $\mathbb{T}_{\varepsilon_{h}}^{+} \cap\left\{t>\varepsilon_{h}\right\}$ and on $\left(0, \varepsilon_{h}\right) \times\left(0, \varepsilon_{h}\right)$, separately.

On $\mathbb{T}_{\varepsilon_{h}}^{+} \cap\left\{s>\varepsilon_{h}\right\}$ we have that $p(t, s)=\delta-s$ and $d(t, s)=t$. Thus

$$
A_{\varepsilon_{h}} \cap\left(\mathbb{T}_{\varepsilon_{h}}^{+} \cap\left\{s>\varepsilon_{h}\right\}\right)=\left\{(t, s) \in \mathbb{T}_{\varepsilon_{h}}^{+}: s \in(\varepsilon, \delta), t \in\left(0, \frac{b \varepsilon_{h}}{2 b-\varepsilon+s}\right)\right\},
$$

and for $(t, s)$ in this set, $\mathbf{u}_{h}(t, s)=\gamma^{-}[\mathbf{u}]\left(\frac{2 b-\varepsilon_{h}+s}{\varepsilon_{h}} t\right)$. Hence

$$
\begin{aligned}
& \mathcal{A}\left(\mathbf{u}_{h}, \mathbb{T}_{\varepsilon_{h}}^{+} \cap\left\{s>\varepsilon_{h}\right\}\right)=\mathcal{A}\left(\mathbf{u}_{h},\left(\mathbb{T}_{\varepsilon_{h}}^{+} \backslash A_{\varepsilon_{h}}\right) \cap\left\{s>\varepsilon_{h}\right\}\right)+\mathcal{A}\left(\mathbf{u}_{h}, A_{\varepsilon_{h}} \cap \mathbb{T}_{\varepsilon_{h}}^{+} \cap\left\{s>\varepsilon_{h}\right\}\right) \\
& =\int_{\varepsilon_{h}}^{\delta} \int_{\frac{b \varepsilon_{h}}{2 b+s-\varepsilon_{h}}}^{\varepsilon_{h}} 1 \mathrm{~d} t \mathrm{~d} s+\int_{\varepsilon_{h}}^{\delta} \int_{0}^{\frac{b \varepsilon_{h}}{2 b+s-\varepsilon_{h}}} \sqrt{1+\left|\dot{\gamma}^{-}\left(\frac{2 b+s-\varepsilon_{h}}{\varepsilon_{h}} t\right)\right|^{2}\left(\frac{\left(2 b+s-\varepsilon_{h}\right)^{2}}{\varepsilon_{h}{ }^{2}}+\frac{t^{2}}{\varepsilon_{h}{ }^{2}}\right)} \mathrm{d} t \mathrm{~d} s \\
& =\varepsilon_{h} \int_{\varepsilon_{h}}^{\delta}\left(\frac{b+s-\varepsilon_{h}}{2 b+s-\varepsilon_{h}}\right) \mathrm{d} s+\int_{\varepsilon_{h}}^{\delta} \int_{0}^{b} \sqrt{\mathcal{O}\left(\varepsilon_{h}^{2}\right)+\left|\dot{\gamma}^{-}(\tau)\right|^{2}\left(1+\mathcal{O}\left(\varepsilon_{h}{ }^{2}\right)\right)} \mathrm{d} \tau \mathrm{d} s \\
& \stackrel{h \rightarrow+\infty}{\longrightarrow} \delta \int_{0}^{a}\left|\dot{\gamma}^{-}(\tau)\right| \mathrm{d} \tau=\pi \rho \delta .
\end{aligned}
$$

13 Again, $\partial_{1}$ and $\partial_{2}$ denote the derivative with respect to the first and the second variable, respectively. 
On $\mathbb{T}_{\varepsilon_{h}}^{+} \cap\left\{t>\varepsilon_{h}\right\}$ we have $p(t, s)=\delta+t$ and $d(t, s)=s$. Thus

$$
A_{\varepsilon_{h}} \cap\left(\mathbb{T}_{\varepsilon_{h}}^{+} \cap\left\{t>\varepsilon_{h}\right\}\right)=\left\{(t, s) \in \mathbb{T}_{\varepsilon}^{+}: t \in\left(\varepsilon_{h}, b\right), s \in\left(0, \frac{(b-t) \varepsilon_{h}}{2 b-2 t+\varepsilon_{h}}\right)\right\},
$$

and for $(t, s)$ in this set, $\mathbf{u}_{h}(t, s)=\gamma^{-}[\mathbf{u}]\left(\frac{2 b-2 t+\varepsilon_{h}}{\varepsilon_{h}} s+t\right)$. Hence

$$
\begin{aligned}
& \mathcal{A}\left(\mathbf{u}_{h}, \mathbb{T}_{\varepsilon_{h}}^{+} \cap\left\{t>\varepsilon_{h}\right\}\right)=\mathcal{A}\left(\mathbf{u}_{h},\left(\mathbb{T}_{\varepsilon_{h}}^{+} \backslash A_{\varepsilon_{h}}\right) \cap\left\{t>\varepsilon_{h}\right\}\right)+\mathcal{A}\left(\mathbf{u}_{h}, A_{\varepsilon_{h}} \cap \mathbb{T}_{\varepsilon_{h}}^{+} \cap\left\{t>\varepsilon_{h}\right\}\right) \\
& =\int_{\varepsilon_{h}}^{b} \int_{\frac{(b-t) \varepsilon_{h}}{2 b-2 t+\varepsilon_{h}}}^{\varepsilon_{h}} 1 \mathrm{~d} t \mathrm{~d} s+\int_{\varepsilon_{h}}^{b} \int_{0}^{\frac{(b-t) \varepsilon_{h}}{2 b-2 t+\varepsilon_{h}}} \sqrt{1+\mid \dot{\gamma}^{-}\left(t+\left.\frac{2 b-2 t+\varepsilon_{h}}{\varepsilon_{h}} s\right|^{2}\left(\left(1-\frac{2 s}{\varepsilon_{h}}\right)^{2}+\frac{\left(2 b-2 t+\varepsilon_{h}\right)^{2}}{\varepsilon_{h}{ }^{2}}\right)\right.} \mathrm{d} s \mathrm{~d} t \\
& =\varepsilon_{h} \int_{\varepsilon_{h}}^{b}\left(\frac{b-t+\varepsilon_{h}}{2 b-2 t+\varepsilon_{h}}\right) \mathrm{d} t+\int_{\varepsilon_{h}}^{b} \int_{0}^{b} \sqrt{\mathcal{O}\left(\varepsilon_{h}^{2}\right)+\left|\dot{\gamma}^{-}(\tau)\right|^{2}\left(1+\mathcal{O}\left(\varepsilon_{h}^{2}\right)\right)} \mathrm{d} \tau \mathrm{d} t \\
& \stackrel{h \rightarrow+\infty}{\longrightarrow} b \int_{0}^{b}\left|\dot{\gamma}^{-}(\tau)\right| \mathrm{d} \tau=\pi \rho b .
\end{aligned}
$$

In order to estimate the area of the graph of $\mathbf{u}_{h}$ on $\left(0, \varepsilon_{h}\right) \times\left(0, \varepsilon_{h}\right)$, is it enough to notice that $\left|\nabla r_{\varepsilon_{h}, 1}\right|=\mathcal{O}\left(\varepsilon_{h}^{-1}\right)$. Therefore

$$
\begin{aligned}
\mathcal{A}\left(\mathbf{u}_{h},\left(0, \varepsilon_{h}\right) \times\left(0, \varepsilon_{h}\right)\right) & =\int_{0}^{\varepsilon_{h}} \int_{0}^{\varepsilon_{h}} \sqrt{1+\left|\partial_{1} \mathbf{u}\left(r_{\varepsilon_{h}}\right)\right|^{2}\left(\left(\partial_{t} r_{\varepsilon_{h}}\right)^{2}+\left(\partial_{s} r_{\varepsilon_{h}}\right)^{2}\right)} \mathrm{d} t \mathrm{~d} s \\
& \leq \varepsilon_{h}^{-1} \int_{0}^{\varepsilon_{h}} \int_{0}^{\varepsilon_{h}} \sqrt{\mathcal{O}\left(\varepsilon_{h}^{2}\right)+\mathcal{O}(1)} \stackrel{h \rightarrow+\infty}{\longrightarrow} 0 .
\end{aligned}
$$

Since by symmetry $\lim _{h \rightarrow+\infty} \mathcal{A}\left(\mathbf{u}_{h}, \mathbb{T}_{\varepsilon_{h}}^{+}\right)=\lim _{h \rightarrow+\infty} \mathcal{A}\left(\mathbf{u}_{h}, \mathbb{T}_{\varepsilon_{h}}^{-}\right)$, (7.12) follows. This implies that $\overline{\mathcal{A}}_{S}(\mathbf{u}, \Omega) \leq 2 \pi \rho(\delta+b)$. Due to our choice of $\rho$, we conclude, as in Example 7.4, that $\overline{\mathcal{A}}_{s}(\mathbf{u}, \Omega)<m\left(D ; \Gamma^{-}[\mathbf{u}], \Gamma^{+}[\mathbf{u}]\right)$.

\section{Non-subadditivity of $\overline{\mathcal{A}}$}

Let us suppose that $\Omega$ and $\mathbf{u}$ satisfy condition I, and choose a map $\mathbf{u}$ of the form

$$
\mathbf{u}(t, s):= \begin{cases}(f(t), 0) & \text { if }(t, s) \in \mathrm{R}^{-}, \\ (f(t), 1) & \text { if }(t, s) \in \mathrm{R}^{+},\end{cases}
$$

where $f \in \operatorname{Lip}([a, b])$ is a piecewise $\mathcal{C}^{1}$-function ${ }^{14}$; for simplicity, we fix $f(a)=0$. Clearly $\gamma^{-}(t)=(f(t), 0)$ and $\gamma^{+}(t)=(f(t), 1)$.

The aim of this section is to prove the following result.

Theorem 8.1 (Non-subadditivity of $\overline{\mathcal{A}}(\mathbf{u}, \cdot))$ Let $\mathbf{u}$ be as in (8.1) for a non-constant function $f$. Then $\overline{\mathcal{A}}(\mathbf{u}, \cdot)$ is not subadditive.

Remark 8.2 It is worth to recall that in [1] it is proven that if $f$ is constant then $\overline{\mathcal{A}}_{s}(\mathbf{u}, \cdot)=$ $\left|D^{s} \mathbf{u}\right|(\cdot)$, and thus $\overline{\mathcal{A}}(\mathbf{u}, \cdot)$ is subadditive.

In order to prove Theorem 8.1, we need some intermediate results: Proposition 8.3, that provides an estimate from above of $\overline{\mathcal{A}}_{s}(\mathbf{u}, \mathrm{R})$, in terms of the area of a suitable semicartesian parametrization (see Fig. 8); Proposition 8.4, where we show that, if $\overline{\mathcal{A}}(\mathbf{u}, \cdot)$ were subadditive, than $\overline{\mathcal{A}}_{s}(\mathbf{u}, \cdot)$ would be forced to coincide with $\left|D^{s} \mathbf{u}\right|(\cdot)$ (see (8.3)); Proposition 8.6, that

${ }_{14}$ That is, there exist $t_{0}=a<t_{1}<\cdots<t_{\ell}<t_{\ell+1}=b$ so that $f \in \mathcal{C}^{1}\left(\left[t_{j}, t_{j+1}\right]\right)$ for $j=0, \ldots, \ell$. 
Fig. 8 The curves

$\Gamma^{-}=\{(t, f(t), 0): t \in[a, b]\}$,

$\Gamma^{+}=\{(t, f(t), 1): t \in[a, b]\}$

for a linear non-constant function $f$, the rectangle $V$, and the sets

$\mathcal{T}_{0}$, and $\mathcal{T}_{1}$ (in this case two

triangles at different heights). The broken dotted curve is the image through the map $\Phi$ of the vertical segment $\mathrm{R} \cap\{t=\bar{t}\}$

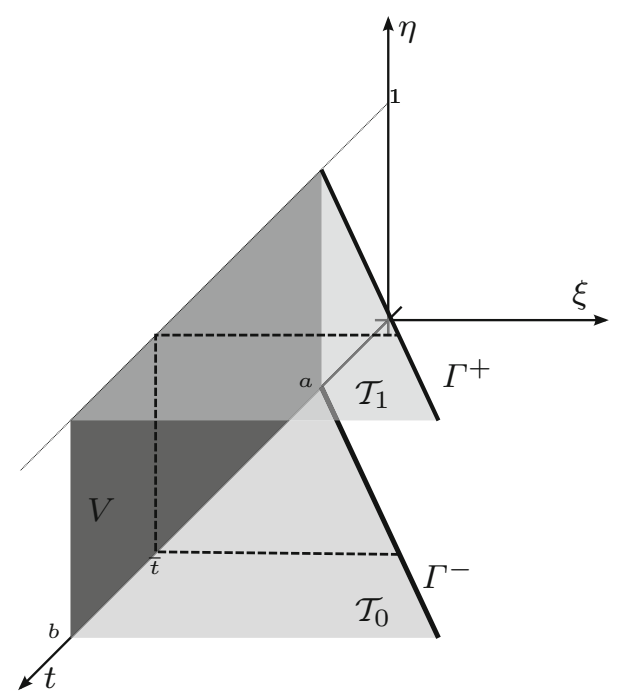

characterizes suitable "vertical" bidimensional currents in $\mathbb{R}^{4}$, whose mass is controlled from above.

We stress that from Proposition 8.4 it follows that there exist coplanar curves $\Gamma^{ \pm}$such that the image of an area-minimizing semicartesian parametrization (if it exists) spanning $\Gamma^{-} \cup \Gamma^{+}$is not planar, see Remark 8.5: this is a consequence of the fact that the area of the rectangle $E$ bounding $\Gamma^{-}$and $\Gamma^{+}$in Fig. 8 can be larger than the sum of the area of its orthogonal projection on the $t \eta$-plane and the areas of the two triangles $\mathcal{T}_{0}$ and $\mathcal{T}_{1}$ (see inequality (8.4)).

Proposition 8.3 Let $\mathbf{u}$ be as in (8.1). Then

$$
\overline{\mathcal{A}}(\mathbf{u}, \mathrm{R}) \leq \int_{\mathrm{R}}|\mathcal{M}(\nabla \mathbf{u})| d t d s+\left|D^{s} \mathbf{u}\right|(\mathrm{R})+\operatorname{lip}(f)(b-a)^{2} .
$$

Proof Since $\left|\mathbf{u}^{+}(t, 0)-\mathbf{u}^{-}(t, 0)\right|=1$ for any $t \in(a, b)$, we have

$$
\left|D^{s} \mathbf{u}\right|(\mathrm{R})=b-a=\mathcal{H}^{2}(V),
$$

where $V$ is the rectangle $V:=[a, b] \times\{0\} \times[0,1] \subset \mathbb{R}_{(t, \xi, \eta)}^{3}$ (see Fig. 8). Let

$$
\mathcal{T}_{0}:=\{(t, \xi, 0): t \in(a, b), \xi \in(0, f(t)) \text { if } 0 \leq f(t), \xi \in(f(t), 0) \text { otherwise }\}
$$

and $\mathcal{T}_{1}:=\mathcal{T}_{0}+(0,0,1)$. We observe that, since $f(a)=0$,

$$
\mathcal{H}^{2}\left(\mathcal{T}_{0}\right)=\mathcal{H}^{2}\left(\mathcal{T}_{1}\right) \leq \frac{\operatorname{lip}(f)}{2}(b-a)^{2},
$$

and hence, if

$$
\Sigma:=\mathcal{T}_{0} \cup V \cup \mathcal{T}_{1},
$$

we have

$$
\mathcal{H}^{2}(\Sigma) \leq\left|D^{s} \mathbf{u}\right|(\mathrm{R})+\operatorname{lip}(f)(b-a)^{2} .
$$


Recalling Proposition 3.1, the result follows if we prove that $\Sigma$ can be parametrized by an injective map $\Phi \in \operatorname{semicart}\left(\mathrm{R} ; \Gamma^{-}, \Gamma^{+}\right)$. This is true, by considering for example the map $\Phi(t, s):=(t, \phi(t, s))$, with $\phi \in H^{1}\left(\mathrm{R} ; \mathbb{R}^{2}\right)$ defined by

$$
\phi(t, s):= \begin{cases}\frac{1-s}{2 / 3}(0,1)+\frac{s-1 / 3}{2 / 3} \gamma^{+}(t) & \text { in } \mathrm{R} \cap\{s \geq 1 / 3\}, \\ \frac{s+1 / 3}{2 / 3}(0,1) & \text { in } \mathrm{R} \cap\{-1 / 3 \leq s<1 / 3\}, \\ -\frac{s+1 / 3}{2 / 3} \gamma^{-}(t) & \text { in } \mathrm{R} \cap\{-1<s<-1 / 3\},\end{cases}
$$

which satisfies $\Phi(R)=\Sigma$.

Proposition 8.4 (Subadditivity and coincidence with $\left|D^{s} \mathbf{u}\right|$ ) Let $\mathbf{u}$ be as in (8.1) for a nonconstant function $f$. If the functional $\overline{\mathcal{A}}(\mathbf{u}, \cdot)$ were subadditive, then

$$
\overline{\mathcal{A}}_{s}(\mathbf{u}, \mathrm{R})=\left|D^{s} \mathbf{u}\right|(\mathrm{R}) .
$$

Proof Fix $\delta \in(0,(b-a) / 2)$ and let $N(\delta) \in \mathbb{N}$ be such that $a+N(\delta) \delta<b \leq a+(N(\delta)+1) \delta$. Define

$$
\begin{array}{ll}
\mathrm{R}_{i}:=[(a+i \delta, a+(i+1) \delta) \cap(a, b)] \times(-1,1), & \text { for } i=0, \ldots, N(\delta), \\
\mathrm{P}_{i}:=\left[\left(a+i \delta-\delta^{2}, a+i \delta+\delta^{2}\right) \cap(a, b)\right] \times(-1,1), & \text { for } i=1, \ldots, N(\delta) .
\end{array}
$$

From Proposition 8.3, applied with $\mathrm{R}_{i}$ and $\mathrm{P}_{i}$ in place of $\mathrm{R}$, it follows

$$
\begin{aligned}
& \overline{\mathcal{A}}\left(\mathbf{u}, \mathrm{R}_{i}\right) \leq \int_{\mathrm{R}_{i}}|\mathcal{M}(\nabla \mathbf{u})| \mathrm{d} t \mathrm{~d} s+\left|D^{s} \mathbf{u}\right|\left(\mathrm{R}_{i}\right)+\operatorname{lip}(f) \delta^{2}, \\
& \overline{\mathcal{A}}\left(\mathbf{u}, \mathrm{P}_{i}\right) \leq \int_{\mathrm{P}_{i}}|\mathcal{M}(\nabla \mathbf{u})| \mathrm{d} t \mathrm{~d} s+\left|D^{s} \mathbf{u}\right|\left(\mathrm{P}_{i}\right)+4 \operatorname{lip}(f) \delta^{4}
\end{aligned}
$$

If $\overline{\mathcal{A}}(\mathbf{u}, \cdot)$ were subadditive, we would get

$$
\begin{aligned}
\overline{\mathcal{A}}(\mathbf{u}, \mathrm{R}) & \leq \sum_{i=0}^{N(\delta)} \overline{\mathcal{A}}\left(\mathbf{u}, \mathrm{R}_{i}\right)+\sum_{i=1}^{N(\delta)} \overline{\mathcal{A}}\left(\mathbf{u}, \mathrm{P}_{i}\right) \\
\leq & \int_{\mathrm{R}}|\mathcal{M}(\nabla \mathbf{u})| \mathrm{d} t \mathrm{~d} s+\left|D^{S} \mathbf{u}\right|(\mathrm{R}) \\
& +\sum_{i=1}^{N(\delta)} \int_{\mathrm{P}_{i}}|\mathcal{M}(\nabla \mathbf{u})| \mathrm{d} t \mathrm{~d} s+\sum_{i=1}^{N(\delta)}\left|D^{s} \mathbf{u}\right|\left(\mathrm{P}_{i}\right)+\mathcal{O}(\delta)+\mathcal{O}\left(\delta^{3}\right) \\
\leq & \int_{\mathrm{R}}|\mathcal{M}(\nabla \mathbf{u})| \mathrm{d} t \mathrm{~d} s+\left|D^{S} \mathbf{u}\right|(\mathrm{R})+\mathcal{O}(\delta) .
\end{aligned}
$$

Since by [1, Theorem 3.7] we have

$$
\overline{\mathcal{A}}(\mathbf{u}, \mathrm{R}) \geq \int_{\mathrm{R}}|\mathcal{M}(\nabla \mathbf{u})| \mathrm{d} t \mathrm{~d} s+\left|D^{s} \mathbf{u}\right|(\mathrm{R}),
$$

the thesis follows letting $\delta \rightarrow 0^{+}$.

Remark 8.5 (Non-planarity) Let us consider a map as in (8.1), for a linear function $f(t)=c(t-a), c>0$ (Fig. 8). In this case the curves $\Gamma^{ \pm}$are coplanar. The map $\Psi \in \operatorname{semicart}\left(\mathrm{R} ; \Gamma^{-}, \Gamma^{+}\right)$defined by

$$
\Psi(t, s):=\left(t, c(t-a), \frac{s+1}{2}\right), \quad t \in(a, b), \quad s \in(-1,1),
$$


parametrizes the rectangle $E$ bounded by $\Gamma^{-}$and $\Gamma^{+}$and the two vertical segments $\{(a, 0, \eta): \eta \in(0,1)\}$, and $\{(b, c(b-a), \eta): \eta \in(0,1)\}$. We have

$$
\mathcal{H}^{2}(E)=(b-a) \sqrt{1+c^{2}}=\int_{\mathrm{R}}\left|\partial_{t} \Psi \wedge \partial_{s} \Psi\right| \mathrm{d} t \mathrm{~d} s .
$$

On the other hand, the sets $\mathcal{T}_{0}$ and $\mathcal{T}_{1}$ built in Proposition 8.4 are two triangles of area $\frac{c(b-a)^{2}}{2}$, so that $\mathcal{H}^{2}(\Sigma)=c(b-a)^{2}+(b-a)$, where $\Sigma$ is defined in (8.2). A simple computation shows that

$$
\mathcal{H}^{2}(E)>\mathcal{H}^{2}(\Sigma)
$$

provided that $b-a \in\left(0, \frac{\sqrt{1+c^{2}}-1}{c}\right)$. Interestingly, this implies that an area-minimizing semicartesian surface (if it exists, recall the beginning of Remark 2.11) spanning two coplanar curves is not necessarily planar.

The next proposition is a modification of [1, Lemma 4.8]. We refer to [1] and [9] for all notations and results concerning cartesian currents.

Proposition 8.6 (Mass lower bound of cartesian two-currents) Let $T=\tau\left(S_{T}, \theta_{T}, \zeta_{T}\right)$ be a 2-dimensional integer rectifiable current with bounded support in $U:=\mathrm{R} \times \mathbb{R}^{2} \subset \mathbb{R}_{(t, s)}^{2} \times$ $\mathbb{R}_{(\xi, \eta)}^{2}$. Denote by $p: U \rightarrow \mathbb{R}_{(t, s)}^{2}$ the orthogonal projection. Suppose that

(i) $\mathcal{L}^{2}\left(p\left(S_{T}\right)\right)=0$,

(ii) $\partial T=\left[\left[\{(t, 0, f(t), 1)\}_{t \in(a, b)}\right]\right]-\left[\left[\{(t, 0, f(t), 0)\}_{t \in(a, b)}\right]\right]$ in $U$, where $f \in \operatorname{Lip}([a, b])$ is piecewise $\mathcal{C}^{1}$.

Then

$$
\text { fnon-constant } \Rightarrow \mathbf{M}_{U}(T)>\int_{a}^{b}|(f(t), 1)-(f(t), 0)| d t=b-a .
$$

Proof Assume by contradiction that $\mathbf{M}_{U}(T) \leq b-a$. Let $\pi: U \rightarrow \mathrm{R} \times \mathbb{R}_{\eta}$ and $q: \mathrm{R} \times \mathbb{R}_{\eta} \rightarrow$ $\mathrm{R}$ be the orthogonal projections, so that $p=q \circ \pi$. Since $T$ has bounded support in $U$, it follows that $\partial\left(\pi_{\sharp} T\right)=\pi_{\sharp}(\partial T)$ (see [9, Sec2.3]) and thus, from assumption (ii), $\partial\left(\pi_{\sharp} T\right)=$ $\left[\left[\{(t, 0,1)\}_{t \in(a, b)}\right]\right]-\left[\left[\{(t, 0,0)\}_{t \in(a, b)}\right]\right]=:[[(a, 0),(b, 0)]] \times[[1]]-[[(a, 0),(b, 0)]] \times[[0]]$ in $\mathrm{R} \times \mathbb{R}_{\eta}$. Since $T$ is rectifiable, also $\pi_{\sharp} T$ is rectifiable, and $\pi_{\sharp} T=\tau\left(S_{\pi_{\sharp} T}, \theta_{\pi_{\sharp} T}, \zeta_{\pi_{\sharp} T}\right)$. Moreover, $S_{\pi \sharp T} \subseteq \pi\left(S_{T}\right)$ and thus, applying the projection $q$ and recalling assumption $(i)$, we get $\mathcal{L}^{2}\left(q\left(S_{\pi_{\sharp} T}\right)\right)=0$. Applying the one-codimensional result in [1, Lemma 4.7], we deduce that $\pi_{\sharp} T=[[(a, 0),(b, 0)]] \times[[0,1]]$. In particular $S_{\pi_{\sharp} T}=(a, b) \times\{0\} \times\{0\} \times[0,1]$, $\theta_{\pi_{\sharp} T}=1$, and $\zeta_{\pi_{\sharp} T}=(1,0,0,0) \wedge(0,0,0,1)$.

Now, we use the assumption on the mass of the current $T$, obtaining

$$
b-a=\mathcal{H}^{2}\left(S_{\pi_{\sharp} T}\right) \leq \mathcal{H}^{2}\left(\pi\left(S_{T}\right)\right) \leq \mathcal{H}^{2}\left(S_{T}\right) \leq \mathbf{M}_{U}(T) \leq b-a ;
$$

hence the above inequalities are indeed equalities and in particular

$$
\pi\left(S_{T}\right) \simeq(a, b) \times\{0\} \times\{0\} \times[0,1]
$$

in the sense of $\mathcal{H}^{2}$. Moreover, since $\mathcal{H}^{2}\left(\pi\left(S_{T}\right)\right)=\mathcal{H}^{2}\left(S_{T}\right)=\mathbf{M}_{U}(T)$, it follows that $\theta_{T}=1$ $\mathcal{H}^{2}$-almost everywhere on $S_{T}$, and $\zeta_{T}=e_{t} \wedge \varepsilon_{\eta}$, where $e_{t}=(1,0,0,0)$ and $\varepsilon_{\eta}=(0,0,0,1)$, see [1, Lemma 4.8].

Thus, if we write any smooth 2 -form $\omega$ compactly supported in $U$ as $\omega:=\omega^{t, s} d t \wedge d s+\omega^{t, \xi} d t \wedge d \xi+\omega^{t, \eta} d t \wedge d \eta+\omega^{s, \xi} d s \wedge d \xi+\omega^{s, \eta} d s \wedge d \eta+\omega^{\xi, \eta} d \xi \wedge d \eta$, 
we have

$$
T(\omega)=\int_{S_{T}} \omega^{t, \eta} .
$$

Let $\alpha:=\alpha^{t} \mathrm{~d} t+\alpha^{s} \mathrm{~d} s+\alpha^{\xi} \mathrm{d} \xi+\alpha^{\eta} \mathrm{d} \eta$ be a smooth 1-form compactly supported in $U$. Then

$$
\partial T(\alpha)=T(d \alpha)=\int_{S_{T}}\left(\partial_{t} \alpha^{\eta}-\partial_{\eta} \alpha^{t}\right) .
$$

On the other hand, from assumption (ii) it follows

$$
\begin{array}{r}
\partial T(\alpha)=\int_{a}^{b}\left[\alpha^{t}(t, 0, f(t), 1)-\alpha^{t}(t, 0, f(t), 0)\right] \\
+f^{\prime}(t)\left[\alpha^{\xi}(t, 0, f(t), 1)-\alpha^{\xi}(t, 0, f(t), 0)\right] \mathrm{d} t .
\end{array}
$$

Now, we choose the 1 -form $\alpha$ such that $\alpha^{t}=0, \alpha^{\eta}=0$, so that the right hand side of (8.5) vanishes, and (8.6) reduces to

$$
\partial T(\alpha)=\int_{a}^{b} f^{\prime}(t)\left[\alpha^{\xi}(t, 0, f(t), 1)-\alpha^{\xi}(t, 0, f(t), 0)\right] \mathrm{d} t .
$$

Since $f$ is not constant and piecewise $\mathcal{C}^{1}$, there exists a non-empty open interval $I \subset$ $(a, b)$ where $f^{\prime}$ is either positive or negative. It is then sufficient to choose $\alpha^{\xi}$ such that $\alpha^{\xi}(t, 0, f(t), 1) \neq \alpha^{\xi}(t, 0, f(t), 0)$ for any $t \in I$, to obtain that the right hand side of (8.6) is nonzero, which is a contradiction.

Now, we are in a position to prove Theorem 8.1

Proof Let us suppose by contradiction that $\overline{\mathcal{A}}(\mathbf{u} ; \cdot)$ is subadditive. As a consequence of Proposition 8.4 and [1, Lemma 3.3], we can select a sequence $\left(\mathbf{u}_{h}\right) \subset \mathcal{C}^{1}\left(\mathrm{R} ; \mathbb{R}^{2}\right)$ converging to $\mathbf{u}$ in $L^{1}\left(\mathrm{R} ; \mathbb{R}^{2}\right)$ and bounded in $L^{\infty}\left(\mathrm{R} ; \mathbb{R}^{2}\right)$, and such that

$$
\mathcal{A}\left(\mathbf{u}_{h}, \mathrm{R}\right) \rightarrow \overline{\mathcal{A}}(\mathbf{u}, \mathrm{R})=\int_{\mathrm{R}}|\mathcal{M}(\nabla \mathbf{u})| \mathrm{d} t \mathrm{~d} s+\left|D^{s} \mathbf{u}\right|(\mathrm{R}) \quad \text { as } h \rightarrow+\infty .
$$

From [1, Theorem 2.6 and Remark 2.4] it follows that the sequence of the graphs [[ $\left.\left.G_{\mathbf{u}_{h}}\right]\right]$ (as currents) of the maps $\mathbf{u}_{h}$ converges weakly in the distributional sense to a cartesian (integer rectifiable) 2-current $T=\tau(S, \theta, \zeta)$, thus with zero boundary, in $U:=\mathrm{R} \times \mathbb{R}^{2}$, decomposable in its regular part $T_{r}=\left[\left[G_{\mathbf{u}}\right]\right]$ and its singular part $T_{S}=\tau\left(S_{S}, \theta, \zeta\right)$; recall that $\mathcal{L}^{2}\left(p\left(S_{s}\right)\right)=0$. By the lower semicontinuity of the mass we have

$$
\mathbf{M}(T) \leq \liminf _{h \rightarrow+\infty} \mathbf{M}\left(\left[\left[G_{\mathbf{u}_{h}}\right]\right]\right)=\overline{\mathcal{A}}(\mathbf{u}, \mathrm{R})
$$

and

$$
\mathbf{M}(T)=\mathbf{M}\left(T_{r}\right)+\mathbf{M}\left(T_{s}\right)=\mathbf{M}\left(\left[\left[G_{\mathbf{u}}\right]\right]\right)+\mathbf{M}\left(T_{s}\right)=\int_{\mathrm{R}}|\mathcal{M}(\nabla \mathbf{u})| \mathrm{d} t \mathrm{~d} s+\mathbf{M}\left(T_{s}\right) .
$$

In addition, the support of $T_{s}$ is bounded in $U$, since $\left(\mathbf{u}_{h}\right)$ is bounded in $L^{\infty}\left(\mathrm{R} ; \mathbb{R}^{2}\right)$, and $\partial T_{s}=-\partial T_{r}=-\partial\left[\left[G_{\mathbf{u}}\right]\right]=\left[\left[\{(t, 0, f(t), 1)\}_{t \in(a, b)}\right]\right]-\left[\left[\{(t, 0, f(t), 0)\}_{t \in(a, b)}\right]\right]$ in $U$. Therefore $T_{s}$ satisfies all hypotheses of Proposition 8.6, hence $f$ has to be constant, providing a contradiction.

Acknowledgments We are very grateful to Gianni Dal Maso for many discussions and advices. 


\section{References}

1. Acerbi, E., Dal Maso, G.: New lower semicontinuity results for polyconvex integrals. Calc. Var. Partial Differ. Equ. 3, 329-371 (1994)

2. Ambrosio, L., Fusco, N., Pallara, D.: Functions of Bounded Variation and Free Discontinuity Problems, Mathematical Monographs. Oxford Univ. Press, Oxford (2000)

3. Bellettini, G., Paolini, M.: On the area of the graph of a singular map from the plane to the plane taking three values. Adv. Calc. Var. 3, 371-386 (2010)

4. Bellettini, G., Paolini, M., Tealdi L.: On the area of a graph of a piecewise smooth map from the plane to the plane with a curve discontinuity. ESAIM: Control, Optimization and Calculus of Variations, 22(1), 29-63 (2016)

5. Dacorogna, B.: Direct methods in the calculus of variations. Applied Mathematical Sciences. vol. 78. Springer, New York (2008)

6. Dal, G.: Maso, integral representation on $\mathrm{BV}(\Omega)$ of $\Gamma$-limits of variational integrals. Manuscr. Math. 30, 387-416 (1979)

7. De Giorgi, E.: On the relaxation of functionals defined on cartesian manifolds. Developments in Partial Differential Equations and Applications to Mathematical Physics. Springer US, pp. 33-38 (1992)

8. Dierkes, U., Hildebrandt, S., Sauvigny, F.: Minimal Surfaces. Grundlehren der mathematischen Wissenschaften, vol. 339. Springer, Berlin (2010)

9. Giaquinta, M., Modica, G., Souček, J.: Cartesian currents in the calculus of variations I. Ergebnisse Math. Grenzgebiete (III Ser). vol. 37, Springer-Verlag, Berlin (1998)

10. Giaquinta, M., Modica, G., Souček, J.: Cartesian currents in the calculus of variations II. Ergebnisse Math. Grenzgebiete (III Ser). vol. 38, Springer-Verlag, Berlin (1998)

11. Giusti, E.: Minimal Surfaces and Functions of Bounded Variation. Birkhäuser, Boston (1984)

12. Goffman, C., Serrin, J.: Sublinear functions of measures and variational integrals. Duke Math. J. 31, $159-178$ (1964)

13. Nitsche, J.C.C.: Lectures on Minimal Surfaces. Cambridge Univ Press, Cambridge (1989) 